\title{
Analysis of Bang-Bang Clock and Data Recovery
}

By

Hazem Abdel-Maguid, B.Sc.

A Master of Engineering thesis submitted to the Ottawa-Carleton Institute for Electrical Engineering Faculty of Engineering

Department of Electronics

Carleton University

Ottawa, Ontario, Canada

September, 2005

(c) 2005 Hazem Abdel-Maguid 


$\begin{array}{ll}\begin{array}{l}\text { Library and } \\ \text { Archives Canada }\end{array} & \begin{array}{l}\text { Bibliothèque et } \\ \text { Archives Canada }\end{array} \\ \begin{array}{l}\text { Published Heritage } \\ \text { Branch }\end{array} & \begin{array}{l}\text { Direction du } \\ \text { Patrimoine de l'édition }\end{array} \\ \begin{array}{l}\text { 395 Wellington Street } \\ \text { Ottawa ON K1A ON4 }\end{array} & \begin{array}{l}\text { 395, rue Wellington } \\ \text { Ottawa ON K1A ON4 } \\ \text { Canada }\end{array}\end{array}$

Your file Votre référence ISBN: 978-0-494-23322-1 Our file Notre référence ISBN: 978-0-494-23322-1

NOTICE:

The author has granted a nonexclusive license allowing Library and Archives Canada to reproduce, publish, archive, preserve, conserve, communicate to the public by telecommunication or on the Internet, loan, distribute and sell theses worldwide, for commercial or noncommercial purposes, in microform, paper, electronic and/or any other formats.

The author retains copyright ownership and moral rights in this thesis. Neither the thesis nor substantial extracts from it may be printed or otherwise reproduced without the author's permission.
AVIS:

L'auteur a accordé une licence non exclusive permettant à la Bibliothèque et Archives Canada de reproduire, publier, archiver, sauvegarder, conserver, transmettre au public par télécommunication ou par l'Internet, prêter, distribuer et vendre des thèses partout dans le monde, à des fins commerciales ou autres, sur support microforme, papier, électronique et/ou autres formats.

L'auteur conserve la propriété du droit d'auteur et des droits moraux qui protège cette thèse. $\mathrm{Ni}$ la thèse ni des extraits substantiels de celle-ci ne doivent être imprimés ou autrement reproduits sans son autorisation.
In compliance with the Canadian

Privacy Act some supporting forms may have been removed from this thesis.

While these forms may be included in the document page count, their removal does not represent any loss of content from the thesis.
Conformément à la loi canadienne sur la protection de la vie privée, quelques formulaires secondaires ont été enlevés de cette thèse.

Bien que ces formulaires aient inclus dans la pagination, il n'y aura aucun contenu manquant.

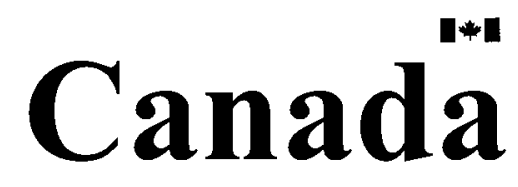




\section{Abstract}

The bang-bang CDR became widely used, however it reveals a lack of theory and mathematical driven analysis of the loop characteristics and parameters, due to having nonlinear phase detector and sampling in the system, which prevents the use of the PLL linear theory in analysis.

The thesis develops a complete set of mathematical equations, inequalities and conditions to explain and characterize both first and second order bang-bang CDR, giving accurate equations for phase step response, frequency step response, sinusoidal operating conditions, and jitter performance as a function of design parameters. A novel analysis describes the stability conditions of the second order loop where the stability factor was defined as a function of the idling order mode, which helps to explain why the loop experiences a wander in the event of a long run pattern of the incoming data stream. The thesis develops novel formulas of both slope overload conditions and signal-to-noise ratio to Gaussian random input jitter as a function of bit-error-rate. 


\section{Acknowledgement}

I am deeply grateful to professor T.A. Kwasniewski of his knowledgeable supervision and constructive criticism. His help, encouragement and guidance have made my study at Carleton University a rich and rewarding experience.

I would especially like to thank my father - mercy be upon him - for his support, and my mother for her continuous encouragement which has been the primary force behind the completion of this work. Finally, I would like to thank my wife for her patience, encouragement and support. 


\section{Intellectual Property}

The information used in this thesis comes in part from a research program of professor T.A. Kwasniewski. The research results appearing in this thesis represents an integral part of the ongoing research program. All research result in this thesis including tables, graphs, and figures but excluding the narrative portion of the thesis are effectively incorporated into the research program and can be used by professor T.A. Kwasniewski for educational and research purpose, including publication in open literature with appropriate credits.

The matter of intellectual property may be pursued cooperatively with Carleton University and professor T.A. Kwasniewski and where and when appropriate. 


\section{Table of Contents}

Chapter 1: Introduction ........................................................................................1

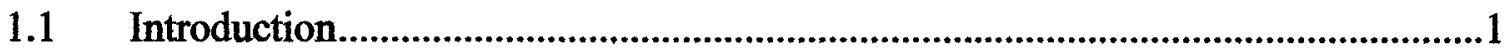

Chapter 2: Overview of IC Architecture of Clock and Data Recovery and Key Jitter Specification 4

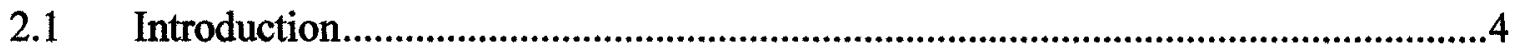

$2.2 \quad$ Linear Clock and Data Recovery .....................................................................6

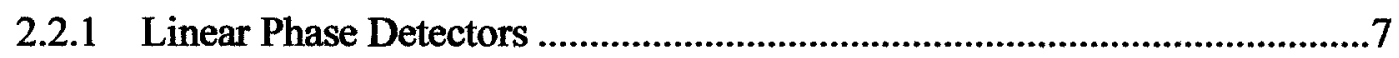

2.2.2 Linear CDR Model Jitter Characteristics.......................................................11

$2.3 \quad$ Non-Linear Clock and Data Recovery ..................................................................13

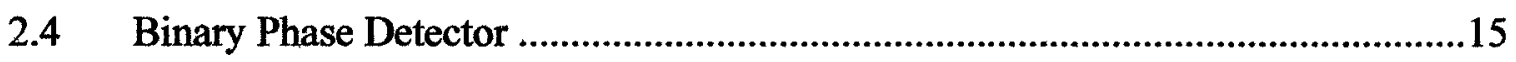

2.5 Clock and Data Recovery Jitter Characterization.................................................16

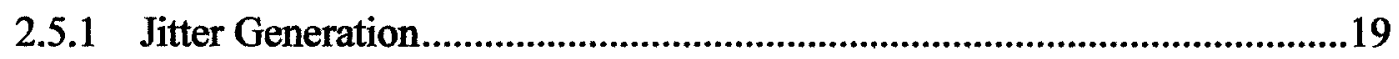

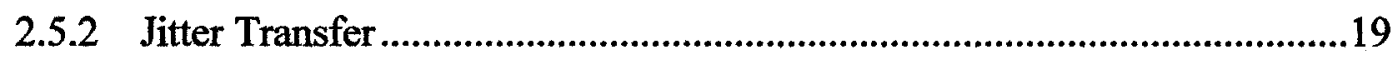

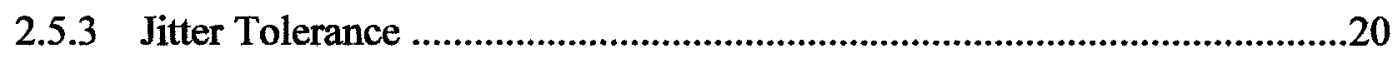

$2.6 \quad$ Literature Review of Non-Linear CDR ..........................................................21

2.6.1 Literature Review of the First Order Loop ................................................22

2.6.2 Literature Review of the Second Order Loop.............................................24

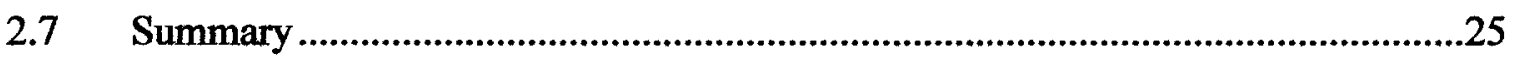

Chapter 3: First Order Bang- Bang Clock and Data Recovery .........................26

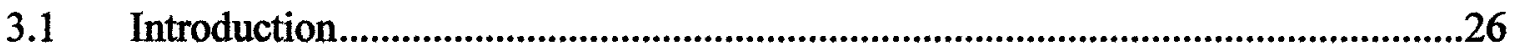

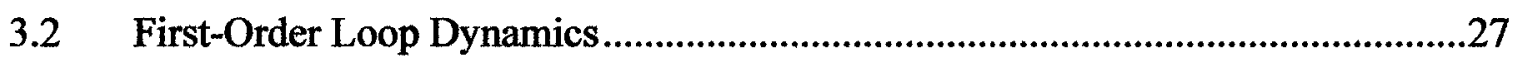

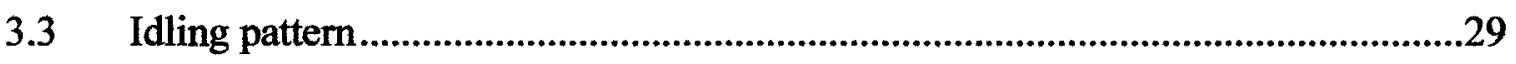


$3.4 \quad$ Slope Overload ...........................................................................................

3.4.1 Slope Overload due to DJ ...........................................................................31

3.4.2 Slope Overload due to RJ .........................................................................32

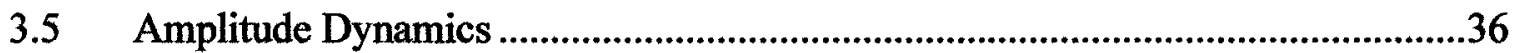

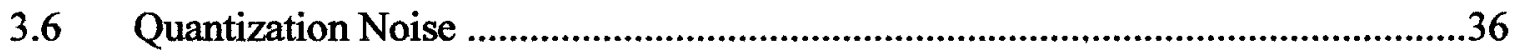

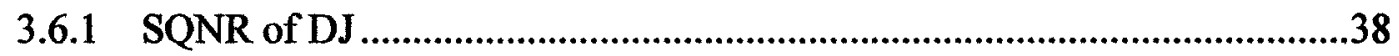

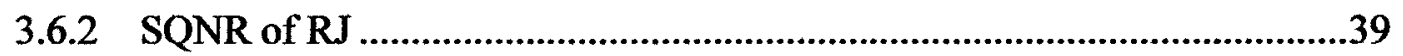

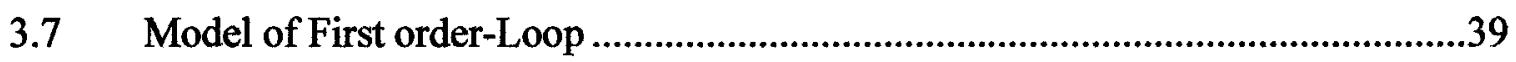

3.8 First order bang-bang linearized model ...............................................................41

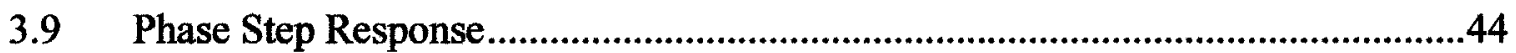

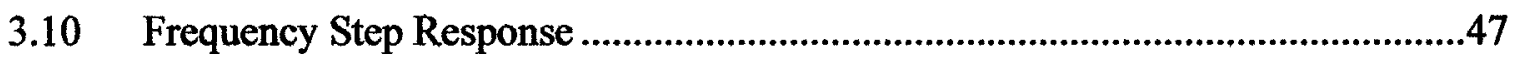

3.11 Sinusoidal Operating Conditions ........................................................................48

$3.12 \quad$ Jitter Analysis of First-Order Loop.....................................................................50

3.12.1 Jitter Transfer................................................................................................51

3.12.2 Jitter Tolerance ……………...........................................................................53

3.12.3 Jitter Generation............................................................................................56

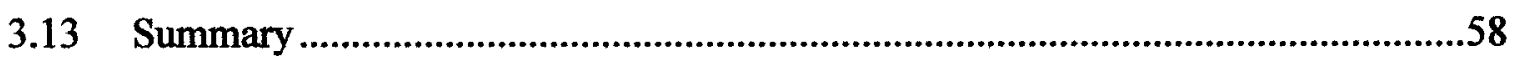

Chapter 4: Second Order Bang- Bang Clock and Data Recovery ......................60

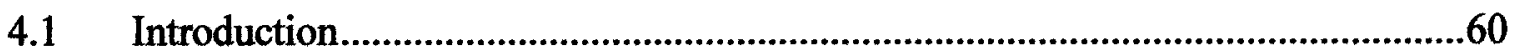

4.2 Second-Order Loop Dynamics .........................................................................61

4.3 Stabilizing the Second Order Loop.......................................................................65

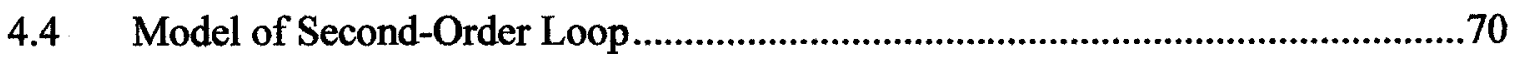

4.4.1 Second Order Bang-bang Linearized Model ..............................................73

4.5 Second Order Loop Slope Overload ...........................................................................76

4.5.1 The Maximum Slope of the Output Phase ....................................................77

vi 
4.5.2 Slope Overload due to DJ .........................................................................78

4.5.3 Slope Overload due to RJ .......................................................................8

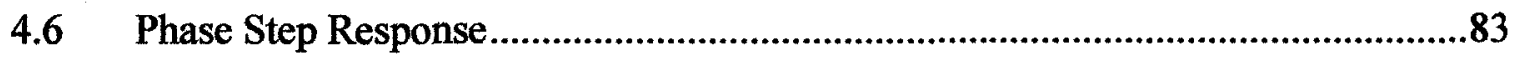

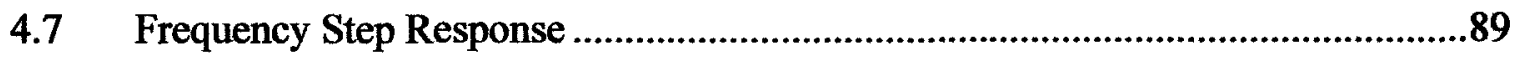

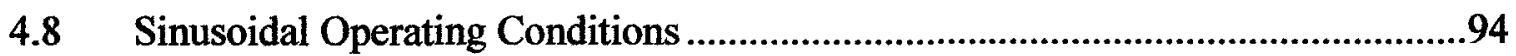

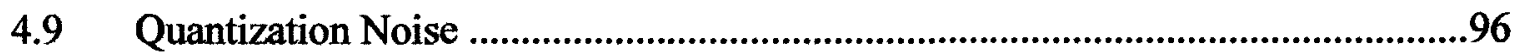

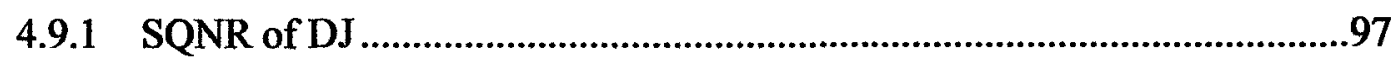

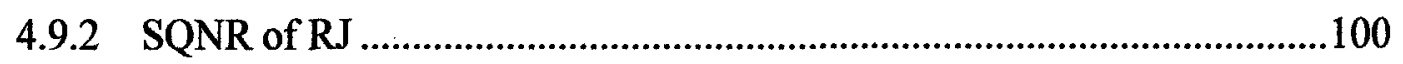

$4.10 \quad$ Jitter Analysis of Second-Order Loop ................................................................102

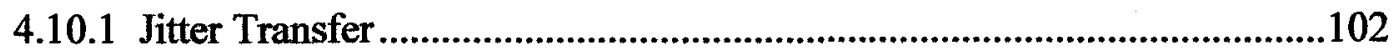

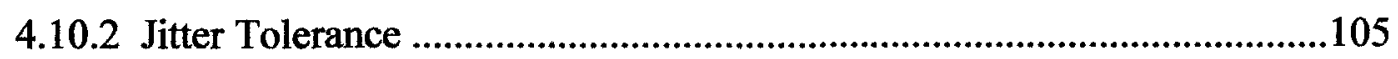

4.10.3 Jitter Generation ......................................................................................108

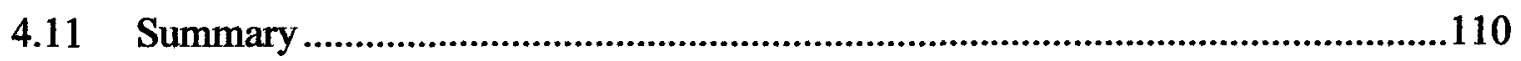

Chapter 5: Conclusion.............................................................................................112

References..............................................................................................................117 


\section{List of Tables}

TABLE 2.1: Truth Table of Alexandar Phase Detector .16

viii 


\section{List of Figures}

Figure 2.1 Optical Receiver 4

Figure 2.2 Typical Linear CDR Architecture.........................................................6

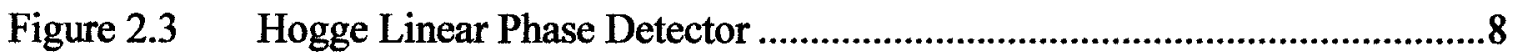

Figure 2.4 Shin and Lee Linear Phase Detector........................................................9

Figure 2.5 Improved Hogge Linear Phase Detector.......................................................10

Figure 2.6 Linear PLL Model ..................................................................................12

Figure 2.7 Non-Linear CDR Architecture.............................................................13

Figure 2.8 Alexander Phase Detector.........................................................................15

Figure 2.9 Eye Diagram with Bathtub Curve.............................................................18

Figure 2.10 SONET OC-192 Jitter Transfer Mask ......................................................20

Figure 2.11 SONET OC-192 Jitter Tolerance Mask ………….........................................21

Figure 2.12 First order bang-bang loop model............................................................22

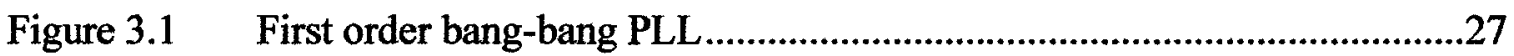

Figure 3.2 Simulated first order loop waveforms correctly tracking the input phase.28

Figure 3.3 Simulated normalized output phase waveform when the first order loop is slope overloaded ..........................................................................................

Figure 3.4 The Linear Model of the Quantizer ........................................................37

Figure 3.5 First order bang-bang loop model based on linear Delta Modulation ........40

Figure 3.6 First order bang-bang loop model based on Delta Sigma Modulation ......41

Figure 3.7 Mathematical mode for analyzing first order loop employing a linear model for quantizer; (a) actual model (b) simplified model ........................42

Figure 3.8 Mathematical model for analyzing first order loop employing a linear model for quantizer; (a) actual model (b) simplified model 


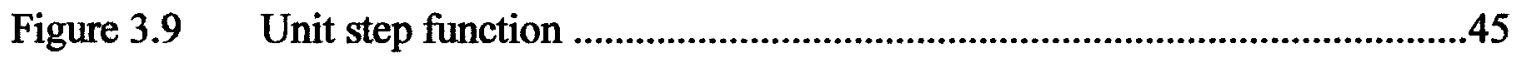

Figure 3.10 Simulated response of first order loop to range of input steps ...................46

Figure 3.11 Simulated the first order loop response to range of bang-bang phase steps when the input is a step in time.

Figure 3.12 Simulated response of the first order loop to a range of input frequency steps

Figure 3.13 Simulated response of first order loop to a sinusoidal jitter with range of amplitudes .50

Figure 3.14 Simulated jitter transfer function of first order loop..................................53

Figure 3.15 First order loop noise model for jitter generation .........................................57

Figure 4.1 Second order Loop Without Phase Lead Correction .................................61

Figure 4.2 Simulated second order loop waveforms when $q(t)$ is series of positive \& negative

Figure 4.3 Simulated second order loop waveforms when is correctly tracking the input

Figure 4.4 Simulated second order loop in idling state.................................................65

Figure 4.5 Second order loop with phase lead correction .............................................66

Figure 4.6 Simulated the effect of applying phase lead correction with minimum stability factor to second order loop

Figure 4.7 Simulated the effect of applying stability factor $<1$ to second order loop.68

Figure 4.8 Simulated the effect of increasing stability factor value on second order loop in its idling state.

Figure 4.9 Second order bang-bang loop model based on linear Delta Modulation...70

Figure 4.10 Second order Bang-Bang loop model based on Delta Sigma Modulation 73

Figure 4.11 Mathematical model for analyzing second order loop employing a linear model for quantizer 
Figure 4.12 Mathematical model for analyzing second order loop employing a linear model for quantizer when the frequency offset is considered .75

Figure 4.13 Simulated normalized output phase waveform when the second order loop has experienced slope overload .76

Figure 4.14 Simulated responses of second and first order loops to phase step .84

Figure 4.15 Simulated response of second order loop to phase step with range of stability factors

Figure 4.16 Simulated response of second order loop to phase step with range of input phase step amplitudes

Figure 4.17 Simulated integral and proportional responses of second order loop to phase step with range of stability factors. .88

Figure 4.18 Simulated integral and proportional response of the second order loop to a frequency step. .90

Figure 4.19 Simulated response of the second order loop to a frequency step with range of stability factors .92

Figure 4.20 Simulated response of the second order loop to a frequency step with range of stability factors

Figure 4.21 Simulated response of second order loop to a sinusoidal jitter with range of stability factors

Figure 4.22 Normalized SQNR of second order loop response to stability factor with range of loop bandwidth when the input is deterministic jitter

Figure 4.23 Normalized SQNR of second order loop response to ratio of $\mathrm{fn} / \mathrm{fjb}$ when the input is random jitter .102

Figure 4.24 Simulated jitter transfer function of second order bang-bang loop ........103

Figure 4.25 Bode plot of jitter tolerance of second order loop .106 
Figure 4.26 Jitter tolerance spectrum of second order loop to deterministic jitter with range of stability factors 


\section{List of Abbreviations}

$\begin{array}{ll}\text { BPD } & \text { binary phase detector } \\ \text { BER } & \text { bit-error rate } \\ \text { BW } & \text { 3-dB bandwidth or corner frequency in rad/sec } \\ \text { CDR } & \text { clock and data recovery } \\ \text { CP } & \text { charge pump } \\ \text { DF } & \text { data transition density } \\ \text { DFmin } & \text { minimum data transition density } \\ \text { DJ } & \text { deterministic jitter } \\ \text { DM } & \text { linear delta modulation } \\ \text { FTR } & \text { frequency transfer function - linearized model } \\ \text { JADR } & \text { jitter amplitude dynamic range } \\ \text { JP } & \text { jitter peaking } \\ \text { JTOL } & \text { jitter tolerance } \\ \text { JTRAN } & \text { jitter transfer function } \\ \text { LPD } & \text { linear phase detector } \\ \text { LPF } & \text { low-pass filter } \\ \text { NRZ } & \text { non-return to zero } \\ \text { NTR } & \text { noise transfer function -linearized model } \\ \text { OC } & \text { optical carrier } \\ \text { OIF } & \text { optical networking forum } \\ \text { PD } & \text { phase detector } \\ & \end{array}$




$\begin{array}{ll}\text { PLL } & \text { phase-locked loop } \\ \text { PTR } & \text { phase transfer function - linearized model } \\ \text { RJ } & \text { random jitter } \\ \text { rms } & \text { root mean square } \\ \text { SONET } & \text { synchronous optical network } \\ \text { SQNR } & \text { signal-to-quantization noise ratio } \\ \text { SQNR } & \text { signal-to-quantization noise ratio } \\ \text { Tc } & \text { phase detector duty cycle } \\ \text { TJ } & \text { total jitter generation } \\ \text { V } & \text { loop gain in rad/sec } \\ \text { VCO } & \text { voltage-controlled oscillator } \\ \text { W } & \text { peak-peak value of dual dirac distribution function } \\ \text { XAUI } & 10 G \text { attachment unit interface }\end{array}$

xiv 


\section{List of Graphical Symbols}

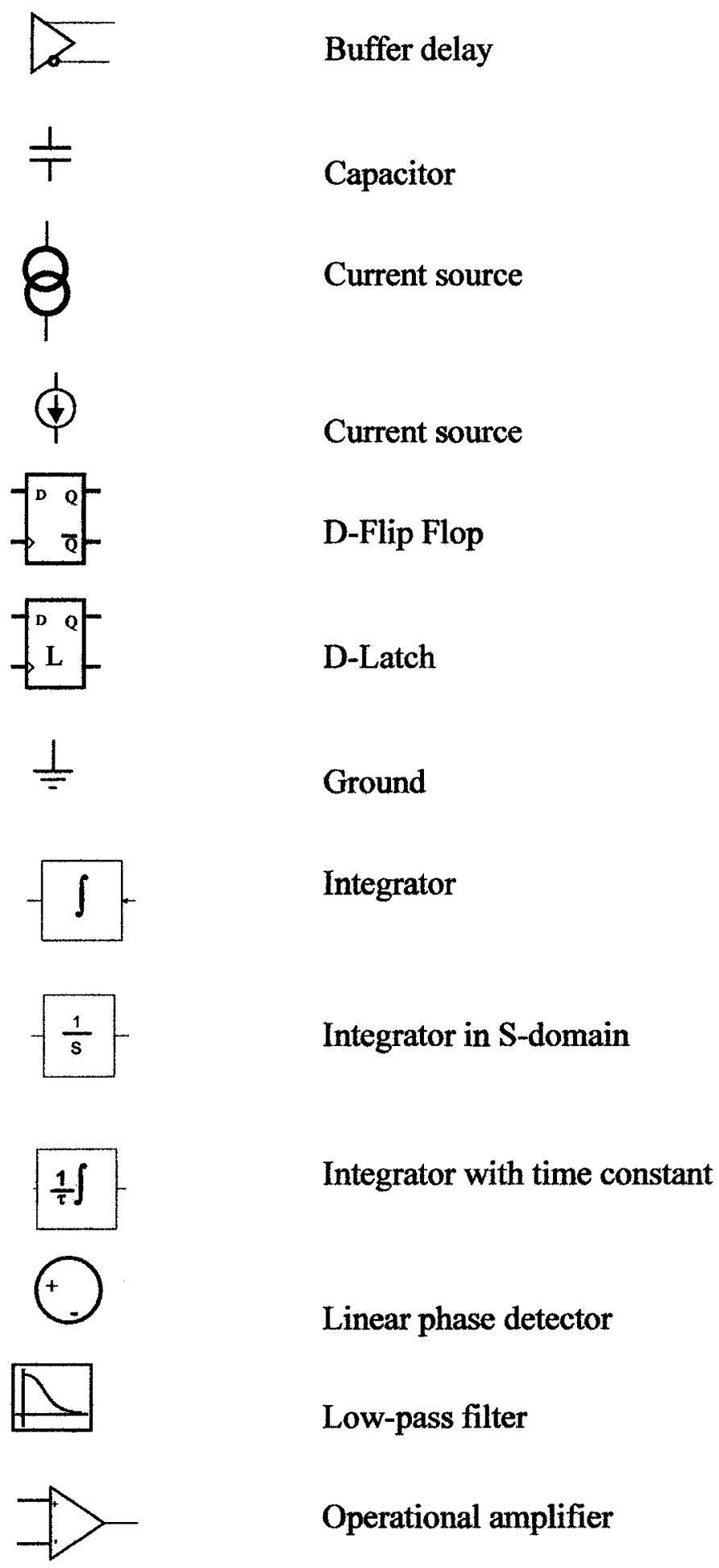

XV 

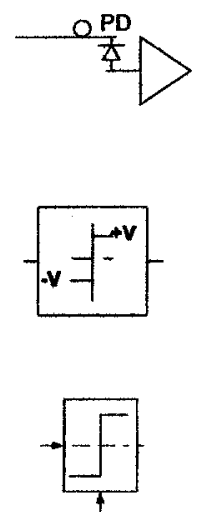

-WMr
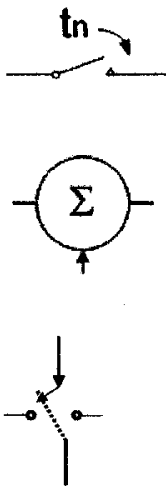

$T$

(2)

DD
Optical receiver

quantizer

quantizer and sampler

\section{Resistor}

Sample and hold

Sum

Switch

VDD

Voltage-controlled oscillator

XOR gate

xvi 


\section{List of symbols}

\begin{tabular}{|c|c|}
\hline$\varepsilon$ & $\mp 1$ \\
\hline$F_{b w}$ & 3-dB bandwidth of linear PLL \\
\hline$T_{a c q}$ & acquisition time \\
\hline$r$ & arbitrary number \\
\hline$K_{H O P}$ & attenuation due to high order poles \\
\hline$\omega_{b b}$ & bang-bang angular frequency step \\
\hline$\left.\omega_{b b}\right|_{J T L}$ & bang-bang angular frequency to satisfy jitter tolerance \\
\hline$\left.\omega_{b b}\right|_{R J}$ & bang-bang angular frequency to satisfy random jitter \\
\hline$f_{b b}$ & bang-bang frequency step \\
\hline$\theta_{b b}$ & bang-bang phase step \\
\hline$\theta_{b b}$ & bang-bang phase step \\
\hline$\left.\theta_{b b}\right|_{J P}$ & bang-bang phase step to satisfy jitter peaking \\
\hline$I_{o}$ & charge pump current \\
\hline erfc & complementary error function \\
\hline$C$ & confidence level. \\
\hline$\zeta$ & damping factor \\
\hline$a$ & decision threshold \\
\hline$K_{q}$ & empirical constant and $0.3<K_{q}<0.9$ \\
\hline$C_{d}$ & empirical constant, $6<C_{d}<18$ \\
\hline
\end{tabular}




\begin{tabular}{|c|c|}
\hline$H(s)$ & frequency step transfer function -linearized model \\
\hline$m_{i}$ & idling mode \\
\hline$m_{\text {idling }}$ & initial idling mode \\
\hline$t_{d}$ & input data stream bit period \\
\hline$\theta_{d}^{\prime}(t)$ & input frequency in $\mathrm{rad} / \mathrm{sec}$ \\
\hline$m_{j}$ & input jitter amplitude \\
\hline$\omega_{j}$ & input jitter angular frequency \\
\hline$f_{j}$ & input jitter frequency \\
\hline$f_{j b}$ & input jitter frequency band \\
\hline$\delta \omega$ & input jitter frequency variation component \\
\hline$\delta f$ & input jitter frequency variation component \\
\hline$\phi\left(t_{n}\right)$ & input jitter phase variation component \\
\hline$s^{2}$ & input jitter power \\
\hline$\phi_{i}$ & input phase \\
\hline$\theta_{d}(t)$ & input phase in rad \\
\hline $\mathrm{f}_{\mathrm{c} 1}$ & input phase lower frequency band \\
\hline $\mathbf{f}_{\mathrm{c} 2}$ & input phase upper frequency band \\
\hline$f_{\text {in }}$ & Instantaneous input frequency \\
\hline$m$ & integer number of sampling time \\
\hline$K_{c p}$ & integration path gain \\
\hline
\end{tabular}

xviii 


$\begin{array}{ll}e r f i n v & \text { inverse of the error function } \\ \Phi_{i} & \text { jitter phase shift } \\ m_{p} & \text { lock-in-frequency time expressed in number of cycles } \\ t_{p} & \text { lock-in-frequency time in sec } \\ C_{o} & \text { loop filter capacitance } \\ R_{o} & \text { loop filter resistance } \\ F(j \omega) & \text { loop filter transfer function } \\ V & \text { loop gain in rad/sec } \\ Q_{B E R} & \text { Marcum's Q function } \\ m_{j \max } & \text { maximum input jitter amplitude } \\ \omega_{j \max } & \text { maximum input jitter angular frequency } \\ J P_{\max } & \text { maximum jitter peaking } \\ f_{j b} & \text { maximum random input jitter frequency component } \\ S Q N R_{\max } & \text { mean square value of input random jitter } \\ \xi_{\max } & \text { maximum sQNR } \\ \theta_{e r r o r}\left(m_{p}\right) & \text { maximum stability factor to avoid cycle slipping } \\ \sigma^{2} & \text { maximum transient phase error } \\ \sigma_{d}^{2} & \text { xix } \\ \left.\xi\right|_{\min } & \text { malue of input random jitter frequency } \\ & \\ & \end{array}$




\begin{tabular}{|c|c|}
\hline$\omega_{n}$ & natural angular frequency \\
\hline \multirow[t]{2}{*}{$\Omega_{0}$} & offset frequency between VCO and incoming data nomi- \\
\hline & nal frequencies in $\mathrm{rad} / \mathrm{sec}$ \\
\hline K & open loop gain \\
\hline$q(\mathbf{t})$ & output of sample and hold block \\
\hline$\phi_{o}$ & output phase \\
\hline$\theta_{v}(t)$ & output phase in rad \\
\hline$\theta_{e}(t)$ & phase error or quantization error in rad \\
\hline$\beta$ & proportional path scaling factor in volt \\
\hline$E(s)$ & quantization error in s-domain \\
\hline$N_{q}^{2}$ & quantization noise power \\
\hline$\delta S Q N R$ & ratio between $2^{\text {nd }}$ order loop SQNR to $1^{\text {st }}$ order loop \\
\hline$S_{h}(s)$ & sample and hold transfer function -linearized model \\
\hline$S_{h}(s)$ & sample and hold transfer function -linearized model \\
\hline$\tau$ & sample point \\
\hline$f_{n}$ & sampling frequency \\
\hline$t_{n}$ & sampling period or update time \\
\hline$S_{n n}(f)$ & spectral density of $\theta_{e}(t)$ \\
\hline$\xi$ & stability factor \\
\hline$\sigma$ & standard deviation of Gaussian distribution \\
\hline$f_{T}$ & technology process speed \\
\hline
\end{tabular}

XX 
$R J_{\text {total }}$

$\Upsilon(t)$

$K_{v}$

$f_{\text {nom }}$

$t_{0}$ total peak-peak random jitter

unit step function

VCO gain in $\mathrm{rad} / \mathrm{sec} / \mathrm{volt}$

VCO nominal frequency in $\mathrm{Hz}$

VCO nominal period time

xxi 


\subsection{Introduction}

With increasing interest in high-speed digital communication, a precise timing recovery mechanism is required. A clock and data recovery circuit (CDR) is a key building block often used as part of the receiver of high-speed serial links and synchronous optical network (SONET) in communication network applications.

The high-speed applications require a clock and data recovery circuit that works at the speed of the incoming data and meets strict jitter and stability requirements. For example, SONET optical carrier (OC-192) applications have incoming data rate of $9.95 \mathrm{Gbps}$ and the peak-to-peak jitter generation requirement is to be less than 10 psec.

These CDRs are based on Phase Locked Loops (PLL), and can be categorized into two groups according to their phase detector type as linear CDR and bang-bang CDR. The bang-bang CDR became widely used, recently, as it offers the highest speed operation close to 0.4 of the technology process speed $\left(f_{T}\right)$ [1], perfect phase alignment between timing and data sampler, as well as ease of design. Nevertheless, the bang-bang CDR suffers 
from the lack of theoretical analysis to explain thoroughly the behavior of the loop dynamics, as well as the lack of equations describing the jitter performance as a function of design parameters.

This thesis presents a detailed explanation of how the bang-bang CDR works with a complete and comprehensive analytical study of both first order and second order loops in terms of behavior, design parameters and jitter performance. Different models for both first order loop and second order loop are also introduced. The work presented in this thesis follows existing analytical models, but their application to Bang-Bang CDR is in most part novel and was not previously reported.

The document is organized in five chapters including this introduction. Chapter 2 covers a review of the architecture of both linear and bang-bang CDR. Different alternative linear phase detector implementations are discussed and compared in terms of their integration technology versus their jitter performance and static phase offset. Then the linear CDR model is briefly reviewed. The bang-bang phase detector implementation is then presented. Jitter characterization, like jitter transfer, jitter peaking, jitter tolerance and jitter generation are defined according to digital communication standards. Literature reviews of first order bang-bang loop and second order loop are discussed respectively.

Chapter 3 presents a framework that describes the characteristics and parameters of first order bang-bang loop based on the linear Delta Modulation system (DM). Idling patterns, dynamic range, and the slope overload are defined to describe the bang-bang first order loop operation. An analytical study of both slope overload and signal-to-noise ratio for both Gaussian random and sinusoidal deterministic jitters are developed. New alterna- 
tive models for first order bang-bang loop CDR are introduced. A complete analytical study is performed through which formulas are developed for phase step response, frequency step response, sinusoidal operating conditions, jitter transfer, jitter peaking, jitter tolerance and jitter generation of first order bang-bang CDR.

Chapter 4 considers an improvement to the first order bang-bang loop, requiring the presence of a phase lead correction. It describes the conditions on how the loop is to be stabilized. Different models of second order bang-bang loop are discussed. A comprehensive mathematical analysis for slope overload and signal-to-noise ratio for both Gaussian and sinusoidal deterministic input jitter signals are developed. Detailed analysis and theory of phase step response, frequency step response, sinusoidal operating conditions, jitter transfer, jitter peaking, jitter tolerance and jitter generation of bang-bang second order are developed.

The final chapter provides a summary of the thesis and suggests recommendations for future work. 


\subsection{Introduction}

With increasing demand on high-speed serial digital data communication applications of synchronous optical networks (SONET), the design of the CDR becomes a challenge. This demand creates a need for ultra high-speed, low power and excellent jitter performance optical receivers where the clock signal is recovered from the non-return-tozero (NRZ) serial data stream by the clock recovery circuit, and then regenerating the data using the recovered clock in the decision circuit. A block diagram of a typical optical receiver is shown in Figure 2.1.

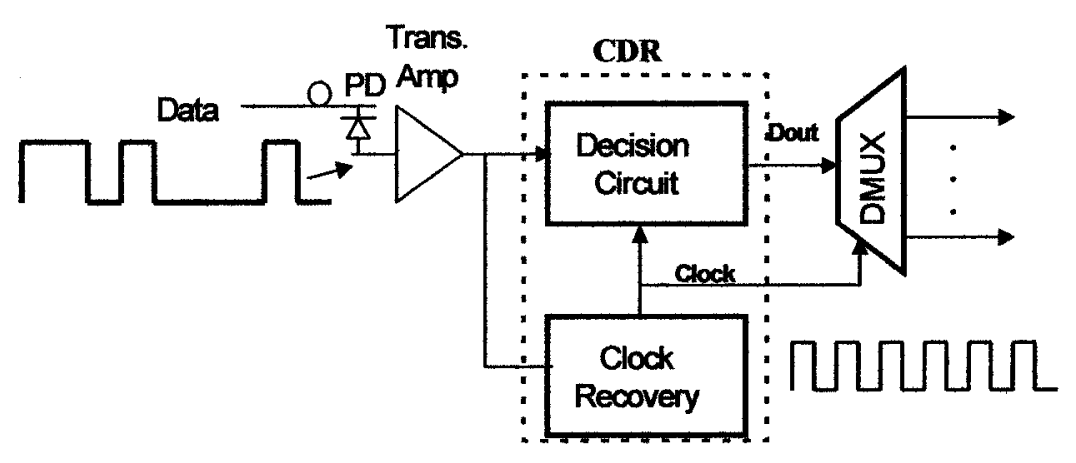

Figure 2.1 Optical Receiver 
Different types of CDR are in common use including LC tuned tank, SAW, digital PLL-based, and analog PLL-based circuits [2]-[9]. However, the LC tuned tank and SAW filter are sensitive to the data pattern and require tuning for their external components to acquire the frequency. The digital PLL experiences a phase build-up at its output. As well, it is not suitable for high frequency operation. The analog PLL is an attractive alternative to utilize in the CDR because of its high integration ability and operation at high frequency.

There are two distinct approaches for implementing a fully integrated PLL based CDR. The first approach utilizes a linear phase detector (LPD), where the average of its output pulses is proportional to the phase error between the timing of data and the clock signal. The second approach is based on a non-linear phase detector that produces binary output representing the direction of the phase error between the timing of data and the clock signal. The speed, technology and jitter requirements are key factors in determining the CDR type.

In this chapter, both linear and non-linear CDR PLL-based will be reviewed. Then they will be compared in terms of their jitter performance and operation at high frequency. In section 2.5 the jitter requirements of high speed CDR for SONET optical receiver will be presented as a practical case study. In the last section, a literature review of the analysis of jitter in the bang-bang CDR will be discussed. 


\subsection{Linear Clock and Data Recovery}

The typical linear CDR architecture is shown in Figure 2.2. The linear phase detector (LPD) compares the phase of incoming data $\phi_{\text {in }}$ to the output phase $\phi_{\text {out }}$ of the clock signal generated from the voltage controlled oscillator (VCO), producing an error pulse that is proportional to the phase difference $\phi$ between the two inputs. The error is then integrated and filtered using a charge pump and low pass filter to produce the control voltage for the VCO.

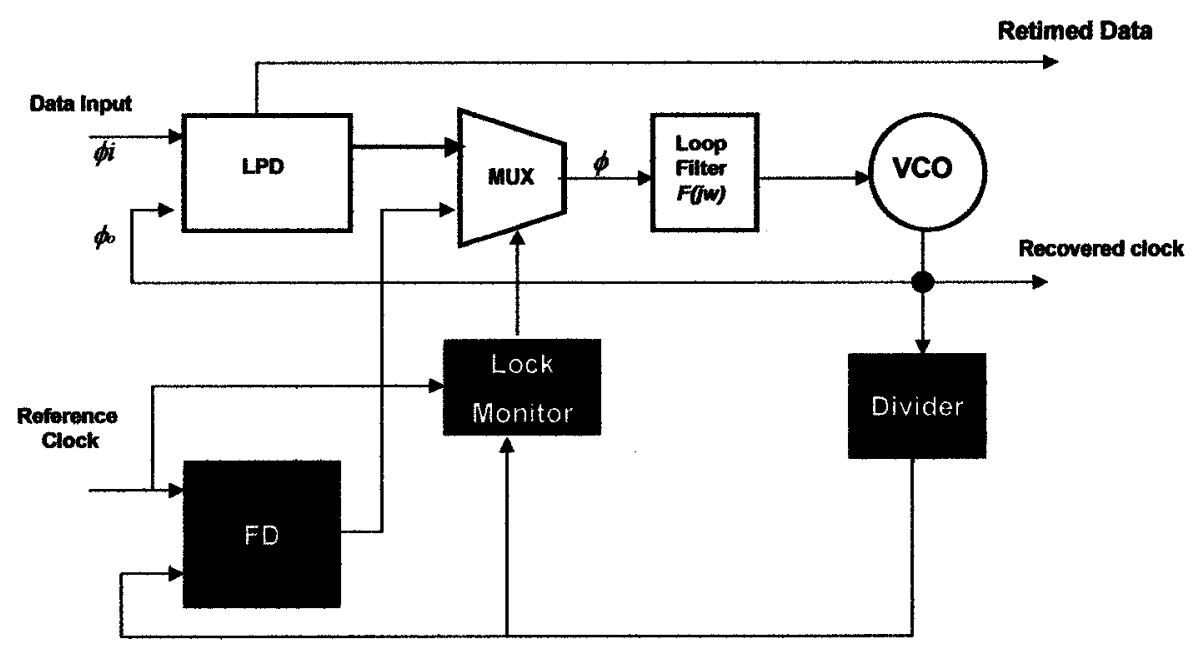

Figure 2.2 Typical Linear CDR Architecture

The transfer function of the loop is defined as

$$
\frac{\phi_{o}(j \omega)}{\phi_{i}(j \omega)}=\frac{K F(j \omega)}{(j \omega-K F(j \omega))}
$$

where $\mathrm{K}$ is the loop gain and $F(j \omega)$ is the loop filter transfer function. 
The clock signal is then used to drive a decision circuit and regenerate the data with better eye opening. The frequency aid circuit is often used for the high speed CDR to boost the VCO frequency closer to the data frequency and prevent false locking states. For signal integrity and reference clock availability from the network backplanes, the frequency aid circuit locks at a relatively low frequency reference clock with respect to data frequency [10].

The acquisition time of second order loop (Tacq) [11] is given by an approximated formula as

$$
T_{a c q}=\frac{\Omega_{0}{ }^{2}}{2 \zeta \omega_{n}{ }^{3}}
$$

where $\Omega_{0}$ is the offset frequency between the VCO frequency and nominal frequency of the incoming data.

\subsubsection{Linear Phase Detectors}

The Hogge phase detector is commonly used as a linear type of phase detector circuit [12]-[14]. The circuit is shown in Figure 2.3. The Hogge detector has two outputs: the first quantizes the phase error and produces fixed width pulses that act as reference output. The second output has variable width pulses that represent the phase difference between the incoming input data and the recovered clock signal. The variable output width pulses and fixed width pulses are integrated and compared to each other to generate the phase error signal. Hence, the phase error is a differential error, which cancels any pattern dependency. The Hogge phase detector's two output pulses have a phase difference of $\pi$ 
radians in the steady state, which produces a non-zero static phase difference and an undesired phase jitter in responding to input signal.

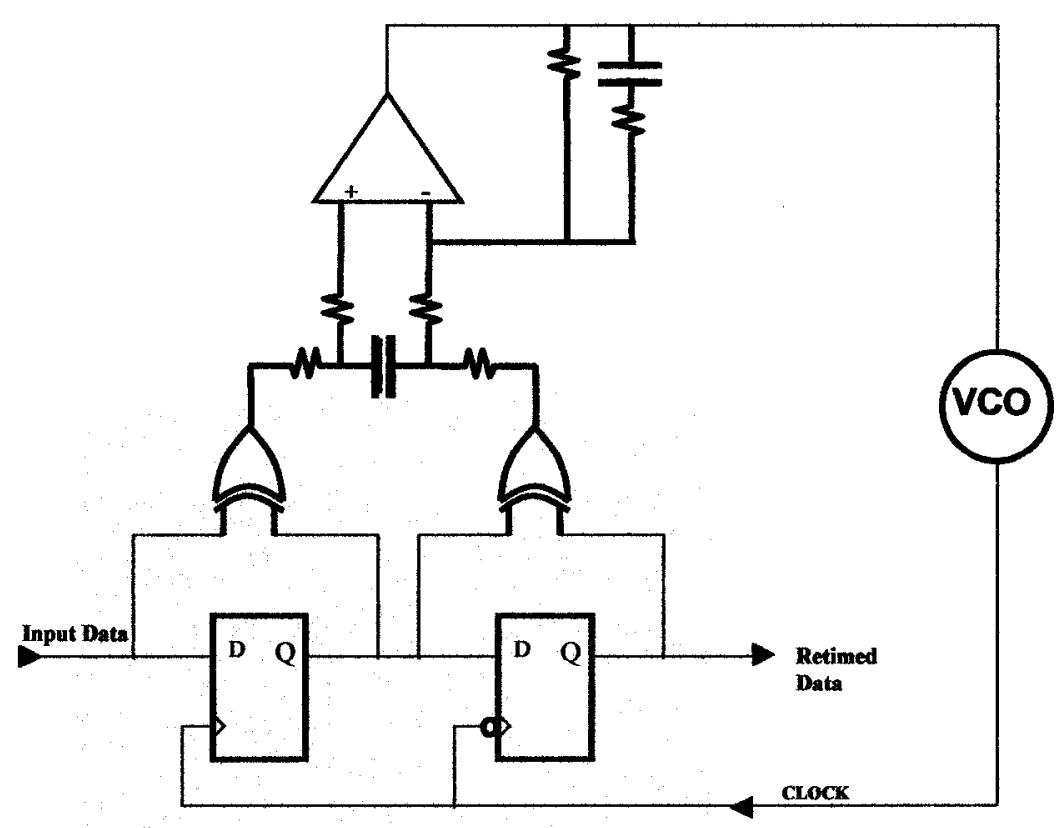

Figare 2.3 Hogge Linear Phase Detector

To function properly, the Hogge circuit requires a relatively small propagation delay through its retiming flip-flop with respect to the bit period. Therefore, the operating speed of the Hogge circuit is limited unless the propagation delay through the retiming flip-flop is compensated. Hogge addressed this problem by balancing the delay through the retiming flip-flop by adding a comparable delay line. Due to the effect of temperature, power supply and process variations, employing the delay line is impractical and undesired for monolithic integrated circuits. In order to meet OC-192 and OC-768 SONET 
application requirements, which require a jitter generation of less than $1 \mathrm{ps} r \mathrm{rs}$, the jitter due to the phase difference of $\pi$ radians must be deleted and the propagation delay must be compensated.

The circuit in Figure 2.4 shows an improved Hogge circuit [15]. Shin and Lee recommended a solution circuit to delete the jitter due to the phase difference. Shin's circuit is based on adding a delay line that is equal to half the bit period of the circuit. The performance of Shin's circuit is critical to any delay line deviation from its original value. Therefore, the Shin's circuit is neither practical nor suitable for monolithic nor high-speed CDR.

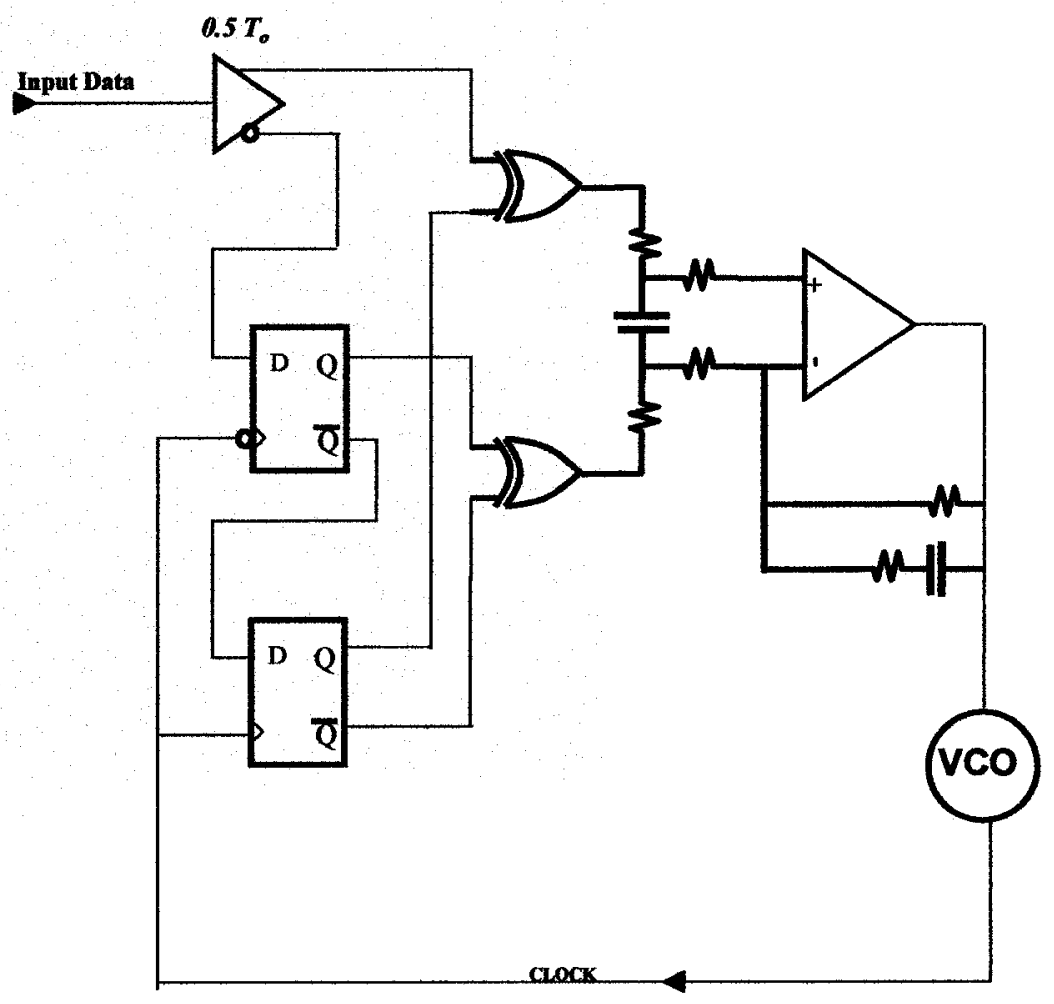

Figure 2.4 Shin and Lee Linear Phase Detector 
Figure 2.5 illustrates a further improvement for Hogge circuit [16]. The circuit is suitable for monolithic integrated circuits that produce zero static phase difference, low jitter and does not utilize a delay line as part of the phase locked loop. The circuit has a better jitter shaping technique that eliminates the jitter resulting from data density variations if the input signal is NRZ data. However, it does not eliminate the cycle-to-cycle jitter, which can be a dominant jitter source especially if the controlled oscillator has minimal time delay response. Therefore, the circuit produces higher peak-to-peak jitter comparable to the Hogge circuit. In addition, like the Hogge circuit, it is not suitable for high-speed operation unless the propagation delay through the retiming flip-flop is compensated.

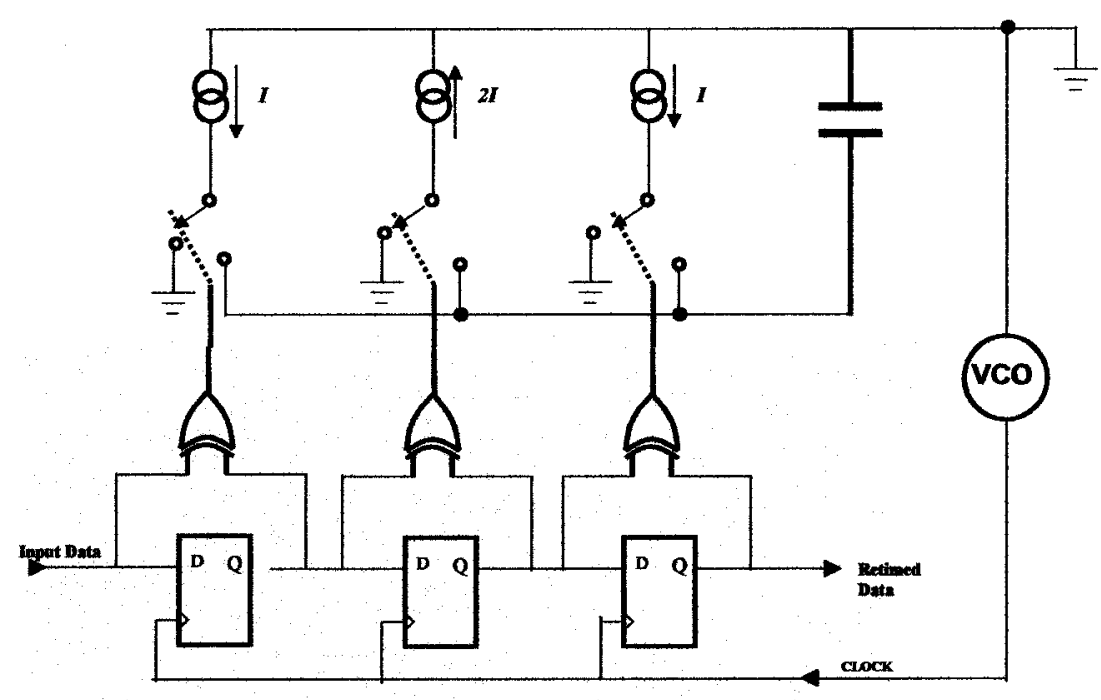

Figure 2.5 Improved Hogge Linear Phase Detector 
A common drawback of using linear phase detectors with setup times that are different from the retiming flip-flops is that the recovered clock will not be intrinsically aligned in the optimum sampling point in the data eye. Another drawback is that the linear phase detector produces narrow pulses proportional to the phase error. These narrow pulses require a technology process speed that exceeds the process speed, which is required to sample the data. Both the setup/hold times and process speed are the limiting factor to realize high speed CDR using a linear phase detector. Alternative techniques for utilizing a high speed CDR using a linear phase detector are reported [17], [18].

\subsubsection{Linear CDR Model Jitter Characteristics}

The jitter characteristics of the linear CDR with Hogge phase detector is analytically predictable using the linear PLL theory. Figure 2.6 shows the equivalent model of the CDR loop. The PLL jitter transfer (JTRAN) function is defined as a single pole lowpass type response with the following parameter definitions [12]:

$$
\begin{gathered}
F_{b w}=\zeta \cdot \frac{\omega_{n}}{\pi} \\
\zeta=\frac{R_{o} \cdot C_{o}}{2} \cdot \omega_{n} \\
\omega_{n}=\sqrt{K_{v} \cdot \frac{2 \cdot I_{o} \cdot D F}{C_{o}}}
\end{gathered}
$$

where

- $F_{b w}$ is the $-3 \mathrm{~dB}$ bandwidth,

- $\zeta$ is the damping factor,

- $\omega_{n}$ is the natural angular frequency,

- $K_{v}$ is the VCO gain in $\mathrm{rad} / \mathrm{sec} / \mathrm{volt}$, 
- $I_{o}$ is the charge pump current,

- $R_{o}$ is the loop filter resistance,

- $C_{o}$ is the loop filter capacitance,

- and $D F$ is the average data transition density.

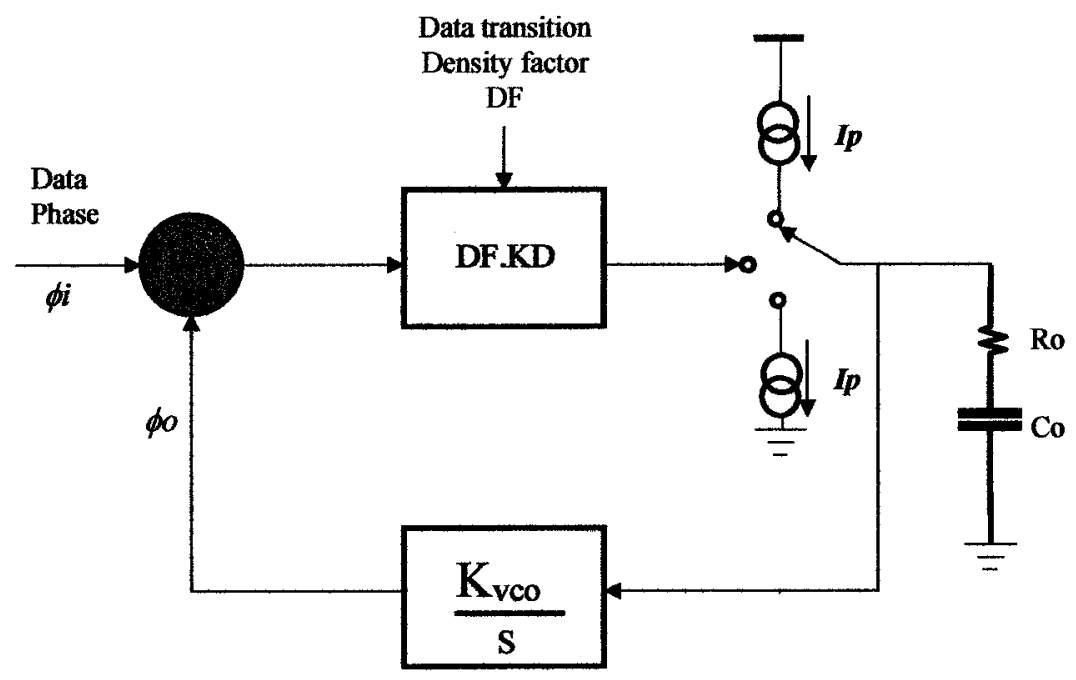

Figure 2.6 Linear PLL Model

Assuming the CDR is correctly tracking the incoming phase, the jitter tolerance (JTOL) can be given by the following function:

$$
\operatorname{JTOL}(s)=\frac{1}{1-J \operatorname{TRAN}(s)}
$$

For the linear PLL to be compliant with SONET OC-192, it has to have a bandwidth $F_{b w}$ of more than $4 \mathrm{MHz}$ for minimum data transition density ( $D F$ min).

The jitter generation (TJ) due to the offset current can be defined as 


$$
T J(p s)=\frac{0.01 \cdot K_{H O P} \cdot \pi \cdot F_{B W}}{D F_{\min }}
$$

where $K_{H O P}$ is the attenuation due to the high order poles in the PLL filter [12]. Jitter generation resulting from the phase noise of the VCO has little impact on the total jitter generation due to the high loop gain and the wide bandwidth of the loop.

\subsection{Non-Linear Clock and Data Recovery}

The non-linear CDR or bang-bang CDR architecture is shown in Figure 2.7. The non-linear phase detector (also called binary phase detector, BPD) produces a pattern of pulses whose mean value is approximately equal to the mean value of the slope of the incoming phase (i.e. the incoming data frequency) over a short period of time.

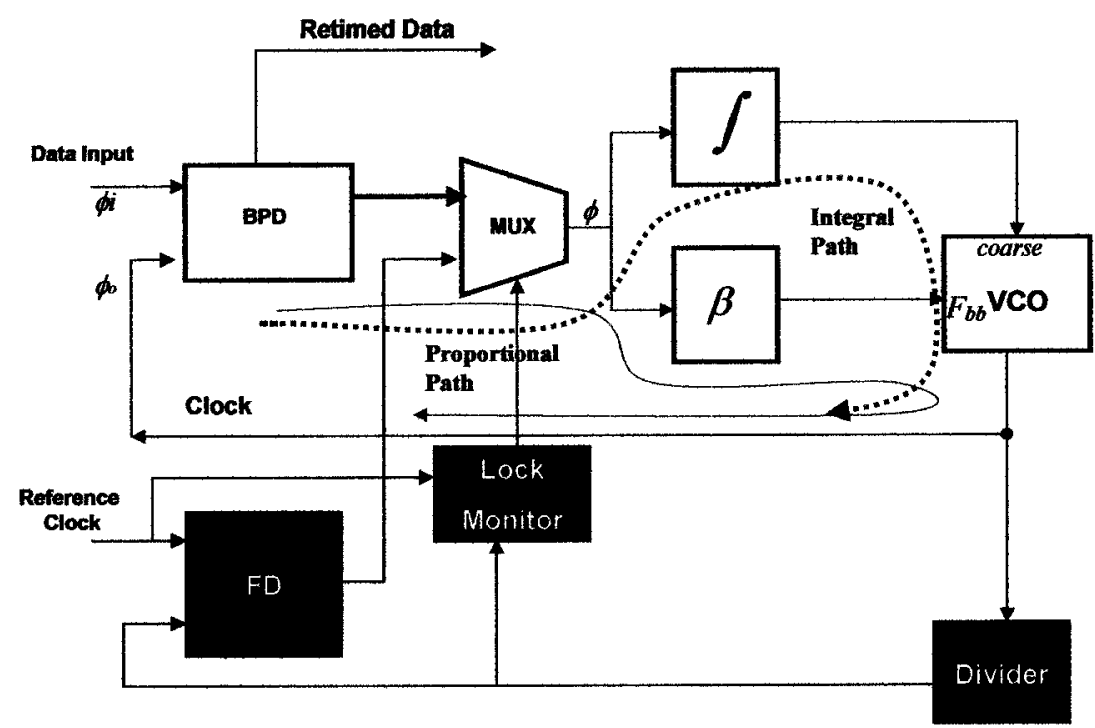

Figure 2.7 Non-Linear CDR Architecture 
The pattern pulses are then evaluated in the binary logic circuit which produces pulses representing the direction of the phase difference between the incoming data and recovered clock. These pulses are fed to integral and proportional loops. The sum of the two loop outputs controls the VCO frequency. The proportional loop is known as bangbang loop and is a fast-correcting loop, while the integral loop is a slow-correcting loop [19]. The bang-bang loop can be considered as a phase tracking loop while the integral loop can be viewed as a frequency-tracking loop.

The phase detector output tends to alternate every data transition. As a result, other than the DC component, the phase detector output spectrum falls outside the bandwidth of the integral loop and can be practically neglected. The integral loop operates solely on the DC component of the phase detector output, setting the VCO center frequency such that the two sideband VCO frequencies, programmed by the bang-bang loop input, will always bracket the frequency of the incoming data signal. The frequency adjustment occurs so slowly that it does not affect the operation of the high frequency bang-bang loop.

Similar to the linear CDR, the recovered clock signal is used to drive a decision circuit and regenerate the data. For a short channel reach, the BPD is often used to regenerate the data. However, for long channel reach, an equalization circuit is needed. 


\subsection{Binary Phase Detector}

The Alexander phase detector circuit (Figure $2.8 \mathrm{a}$ ) is commonly used as a binary phase detector in ultra high speed CDR [20]-[25]. The Alexander phase detector evaluates three data samples and generates a binary output with respect to the direction of the VCO clock phase (lag or lead) [26]. Two samples are used to sample the middle of the eye in two consecutive bits. The third sample is used to sample the eye transition (Figure 2.8b).

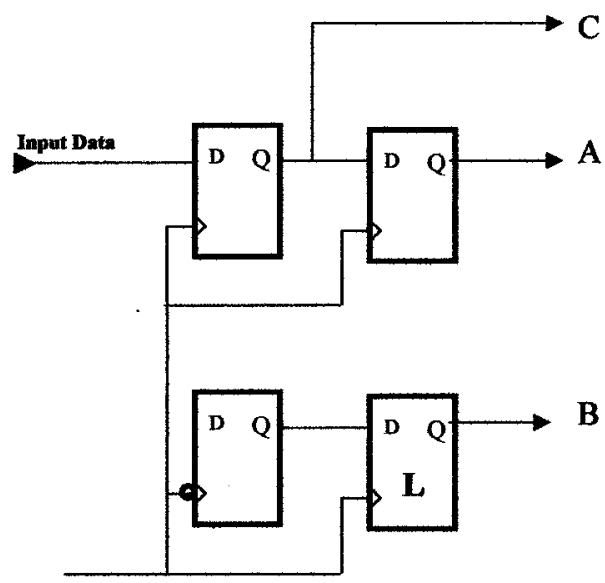

(a)

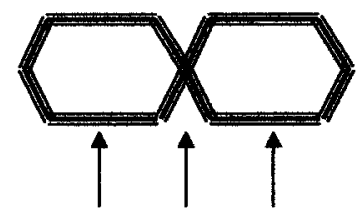

A B C

Figure 2.8 Alexander Phase Detector

The three samples are then aligned and fed to a binary logic circuit to produce digital outputs, UP and DOWN, to signal if the data is early or late with respect to the VCO clock. The UP and DOWN can be programmed to create a hold state during the absence of data transition, or for an invalid state. Table 2.1 is a truth table that presents the binary logic of the UP and DOWN signals. The UP and DOWN signals are used to program the VCO frequency to hunt between three frequencies, $f_{0}-f_{b b}, f_{o}, f_{0}+f_{b b}$, where $f_{o}$ is the 
nominal VCO frequency and $f_{b b}$ is an offset frequency from the nominal VCO frequency.

In steady state, the output of the phase detector is a quasi-random string of " 1 's" and " 0 ' $s$ " which will force the VCO to switch between its two sideband frequencies, causing ramping up or down in phase and tracking the incoming data phase. The average output of the binary phase detector is proportional to the frequency variation, unlike the conventional linear phase detector in which the average output is proportional to the phase variation.

TABLE 2.1: Truth Table of Alexandar Phase Detector

\begin{tabular}{|l|l|l|l|l|l|l|}
\hline Data State & A & B & C & UP & DOWN & Frequency \\
\hline no data & 0 & 0 & 0 & 0 & 0 & $f_{o}$ \\
\hline transition 0 to 1 -late & 0 & 0 & 1 & 0 & 1 & $f_{0}-f_{b b}$ \\
\hline not a valid state & 0 & 1 & 0 & 0 & 0 & $f_{o}$ \\
\hline transition 0 to 1 -early & 0 & 1 & 1 & 1 & 0 & $f_{0}+f_{b b}$ \\
\hline transition 1 to 0 -early & 1 & 0 & 0 & 0 & 1 & $f_{0}-f_{b b}$ \\
\hline not a valid state & 1 & 0 & 1 & 0 & 0 & $f_{o}$ \\
\hline transition 1 to 0 -late & 1 & 1 & 0 & 1 & 0 & $f_{0}+f_{b b}$ \\
\hline no date & 1 & 1 & 1 & 0 & 0 & $f_{o}$ \\
\hline
\end{tabular}

\subsection{Clock and Data Recovery Jitter Characterization}

Jitter is defined as a short-term variation of a digital signal's significant instant from its original position in time [27]. Standards such as SONET and Optical Networking Forum (OIF) impose strict requirements on jitter performance for high-speed clocks and data recovery circuits. Low frequency jitter is tracked by the clock recovery circuit, and does not directly change the timing position of the sampling point within the bit interval, 
and hence does not degrade the bit error rate $(B E R)$. Jitter setting beyond the clock recovery circuit bandwidth (high-frequency jitter) is not tracked and directly affects the timing position of the sampling point and degrades the BER.

Jitter is considered comprised of both bonded high probability deterministic jitter (DJ) and unbounded low probability random jitter $(R J)$. The RJ is defined in terms of a Gaussian distribution with standard deviation $\sigma$, while the DJ is defined as a Dual Dirac distribution function in terms of its peak-to-peak value $(W)$ [28]. The total jitter (TJ) model is defined as

$$
T J(\tau, W, \sigma)=\frac{1}{2 \sqrt{2 \pi}} \cdot \frac{1}{\sigma} \cdot\left[e^{\left[-\left[\frac{\left(\tau-\frac{W}{2}\right)^{2}}{2 \sigma^{2}}\right]\right.}+e^{-\left[\frac{\left(\tau+\frac{W}{2}\right)^{2}}{2 \sigma^{2}}\right]}\right]
$$

The total peak-to-peak random jitter $\mathrm{RJ}_{\text {total }}$ grows with measurement time and therefore depends on the BER. It can be defined as

$$
R J_{\text {total }}=2 \cdot Q_{B E R} \cdot R J
$$

where $Q_{B E R}$ is Marcum's Q function and is defined as

$$
Q_{B E R}=\sqrt{2} \cdot \operatorname{erfinv}(2 \cdot(1-B E R)-1)
$$


where erfinv is the inverse of the error function (e.g. $\mathrm{Q}_{\mathrm{BER}}=7.0345$ for $\mathrm{BER}=10^{-12}$ ). The deterministic jitter is bounded in amplitude and is the sum of duty cycle distortion, data dependent sinusoidal, periodic and bounded jitters. The random jitter is unbounded in amplitude and results from the phase noise random process mechanism typically found in the VCO.

The BER is measured when the sample point, $\tau$, is swept between two eye zero crossings. The probability of bit error, as a function of the sample point timing position, is defined as BER bathtub curve. Figure 2.9 shows a graphical representation of an eye diagram with ideal sampling point and its bathtub curve.

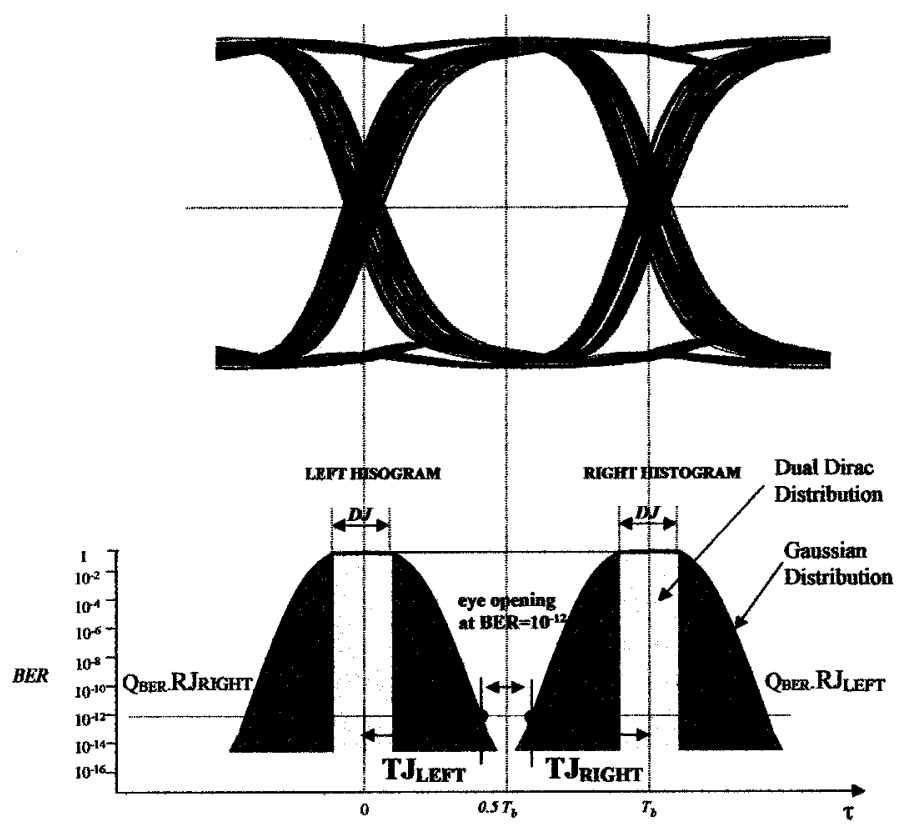

Figure 2.9 Eye Diagram with Bathtub Curve 
The clock and data recovery circuit design is critical to three jitter criteria; jitter generation, jitter transfer, and jitter tolerance.

\subsubsection{Jitter Generation}

The jitter generation (TJ) is defined as the total output jitter with absence of input jitter at the input of the CDR. The jitter generation is the sum of intrinsic deterministic jitter and intrinsic random jitter at a given BER. For example, the 10G Attachment Unit Interface (XAUI) requires the total jitter to be measured at $\mathrm{BER}=10^{-12}$. A clock recovery circuit with tri-state phase detector cancels any correlated jitter to the data pattern on the assumption that the frequency drift due to the tri-state is negligible, and therefore the peak-to-peak jitter can be calculated using $101010 \ldots$ pattern.

\subsubsection{Jitter Transfer}

Jitter transfer is defined as the ratio of output jitter to the sinusoidal input jitter. The jitter transfer requirement is to limit the amount of jitter at the input to be transferred to the output electrical signal. For example, the SONET specifications for an OC-192 requires a low pass jitter transfer function with bandwidth less than $120 \mathrm{kHz}$ and with maximum peaking of $0.1 \mathrm{~dB}$. The jitter peaking is the dominant contributor for the accumulation jitter in the repeater applications. Figure 2.10 illustrates the SONET OC-192 jitter transfer mask. The clock recovery circuit (receiver) should have a jitter peaking of less than $\quad 0.1 \mathrm{~dB}$, while the transmitter side must meet both jitter bandwidth and jitter peaking requirements. Similar to the jitter generation, the data pattern to perform the jitter transfer test is $101010 \ldots$. 


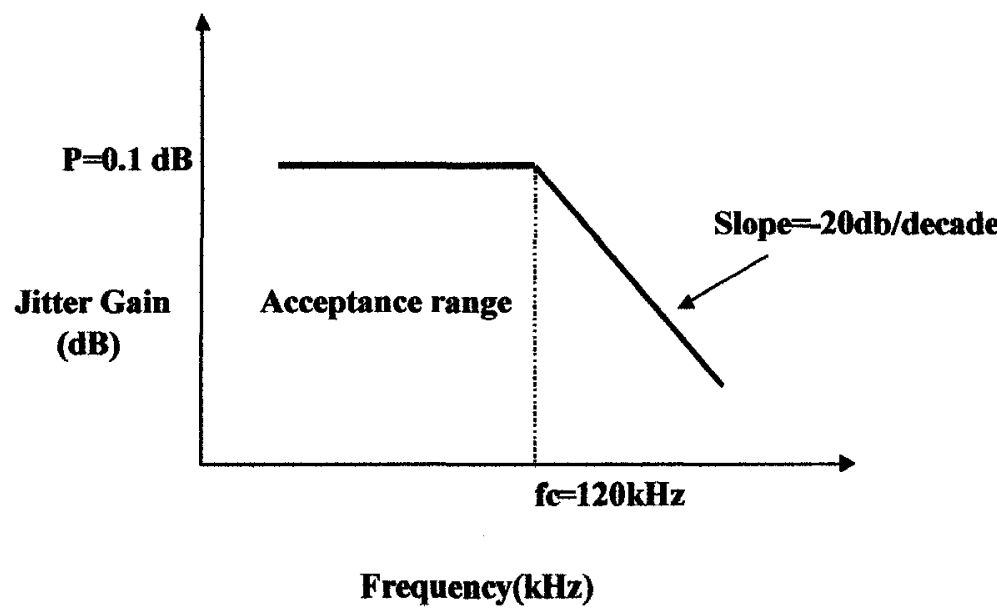

Figure 2.10 SONET OC-192 Jitter Transfer Mask

\subsubsection{Jitter Tolerance}

Jitter tolerance is an important measurement to characterize the receiver's (i.e. CDR's) ability to tolerate jittery inputs and yet recover error-free data. This test is performed by feeding a well controlled jittery sinusoidal signal to a CDR and measure the BER at the output. As the source jitter signal is swept in amplitude and frequency, the change in BER is measured. For example, in SONET, jitter tolerance is the peak-to-peak amplitude of jitter that causes BER $=10^{-10}$. Figure 2.11 illustrates the SONET OC-192 jitter tolerance mask. 


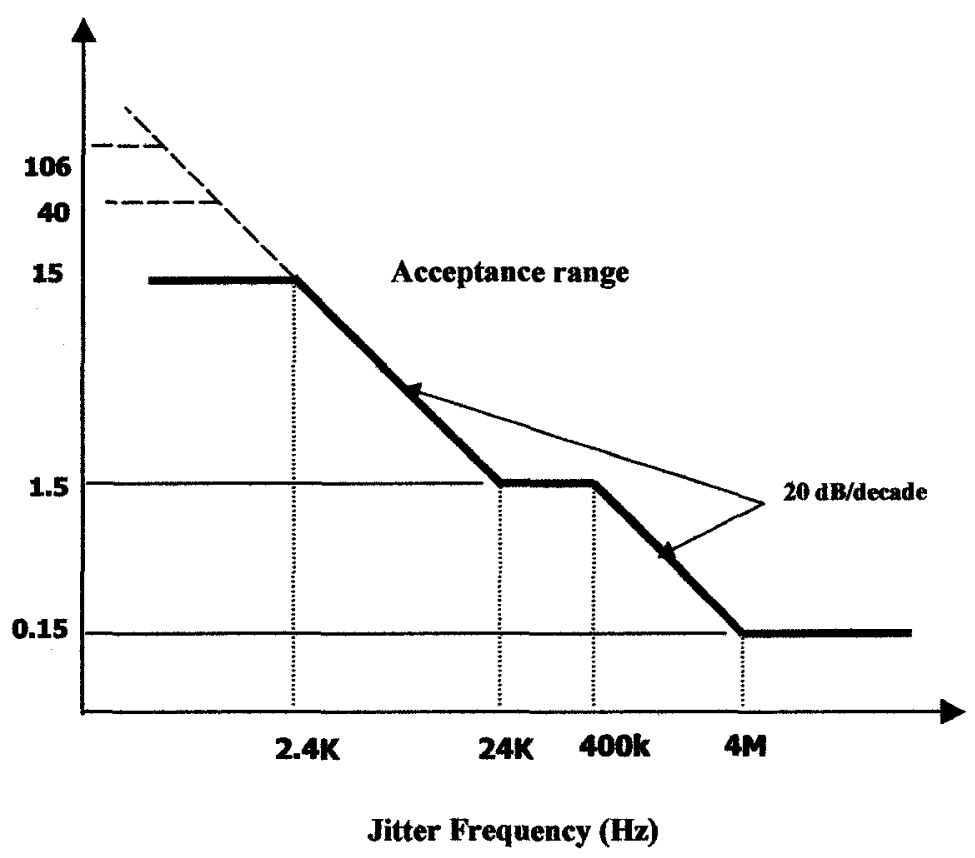

Figure 2.11 SONET OC-192 Jitter Tolerance Mask

\subsection{Literature Review of Non-Linear CDR}

Recently, non-linear CDR has become an attractive option for many clock and data recovery systems where a superior performance is needed at very high speed bit rates. A scanning of the literature on non-linear clock and data recovery PLL loop dynamic theory reveals a lack of comprehensive analysis of the loop which fully accounts for the non-linearity of the system. An exception is the work by Walker [1], which illustrates the influence of non-linear phenomena on loop behavior for the first and second order loops. Another work by Jri Lee [29][30] discusses an approximated formula for jitter performance for the non-linear CDR and M. Ramezani [31]-[33] discusses the lock-in-frequency 
range, lock-in phase and jitter performance of the second order loop. Thus, very few analytical design aids that predict nonlinear behavior can be found for nonlinear CDR [32][37]. Many researchers have observed experimental phenomena for which, to date, no analytical explanations exit. Some examples include the instability of second order loop, jitter analysis for the loop dynamics and wander analysis.

\subsubsection{Literature Review of the First Order Loop}

To our knowledge, the research by Walker [1] was the only one to explain how the bang-bang loop CDR works. Walker analyzed the loop dynamics of the first order bangbang PLL by modeling the phase detector with a binary-quantizer model as shown in Figure 2.12 , where the binary quantizer limits the phase error of the loop at each sampling time $\left(t_{n}\right)$. The phase difference between the data phase $\theta_{d}\left(t_{n}\right)$ and the VCO phase $\theta_{v}\left(t_{n}\right)$ at $\mathrm{n}^{\text {th }}$ sampling time $t_{n}$ is denoted by $\theta_{e}\left(t_{n}\right)$. The incoming data signal frequency is different from the VCO frequency by $\delta f$ and has a zero mean phase jitter of $\phi_{d}(t)$. The change in VCO frequency from its normal frequency $\left(f_{\text {nom }}\right)$ due to the error signal is defined as $f_{b b}=\beta K_{v}$, where $\beta$ is the attenuator for the phase error signal and $K_{v}$ is the VCO gain.

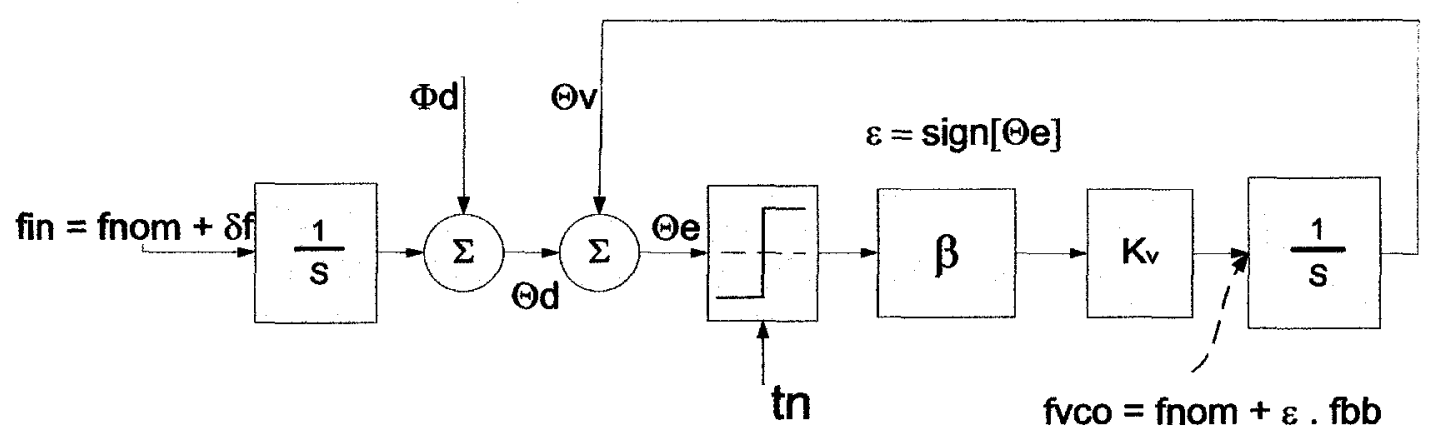

Figure 2.12 First order bang-bang loop model 
A set of discrete time difference equations were derived to describe the loop dynamics as

$$
\begin{gathered}
\theta_{d}\left(t_{n}\right)=\theta_{d}(0)+2 \pi \delta f t_{n}+\phi_{d}\left(t_{n}\right) \\
\theta_{v}\left(t_{n+1}\right)=\theta_{v}\left(t_{n+1}\right)+\varepsilon_{n} \theta_{b b} \\
\varepsilon_{n}=\operatorname{sign}\left(\theta_{d}\left(t_{n}\right)-\theta_{v}\left(t_{n}\right)\right)
\end{gathered}
$$

where

$$
\theta_{b b}=2 \pi \frac{f_{b b}}{f_{\text {nom }}}
$$

Walker discussed both phase step and frequency step responses. However, the timing model used for the simulation is not accurate and contradicts the duty cycle equation of the phase detector. Walker defined the phase detector duty cycle $(T c)$ as

$$
T_{c}=\left(\frac{1}{2}+\frac{\delta f}{2 f_{b b}}\right)
$$

where $T c=1$ when $\delta f \geq f_{b b}$ and $T c=0$ when $\delta f \leq-f_{b b}$. The loop will stay in lock as long as the $f_{b b}$ brackets the input signal frequency. To overcome the difficulty of the analysis, a clock signal is assumed as input, instead of NRZ data stream. A brief discussion is provided with no further analysis for the consequence of an input of NRZ data stream. 


\subsubsection{Literature Review of the Second Order Loop}

Walker [1] developed a model for the second order loop bang-bang and analyzed the loop dynamics of the second order bang-bang PLL by adding an extra integrator, $\frac{1}{\tau s}$, to the loop filter between the binary quantizer and the VCO. He then defined a simplified equivalent model, where the inner loop is a first order loop for phase tracking and the outer loop is a frequency-tracking loop. It is clear that the two loops are not exactly equivalent, since some signals do not match and do not have a physical meaning. Unlike the first order loop, Walker developed an inaccurate set of discrete time difference equations describing the loop dynamic that leads to erroneous conclusions and results.

The stability factor of the second order loop was first defined as

$$
\xi \equiv \frac{\Delta \theta_{\text {proportional }}}{\Delta \theta_{\text {integral }}}=\frac{2 \beta \tau}{t_{\text {update }}}
$$

where:

- $t_{\text {update }}=\frac{1}{f_{\text {nom }}}$, with the assumption that the system has uniform sampling times

- $\Delta \theta_{\text {proportional }}$ is the phase change in one time update due to the bang-bang (proportional) branch. It is defined as

$$
\Delta \theta_{\text {proportional }} \equiv \Delta \theta b b=\beta K_{v} t_{\text {update }}
$$

- $\Delta \theta_{\text {integral }}$ is the loop phase change in one update time due to the integral branch and is defined as

$$
\Delta \theta_{\text {integral }} \equiv \Delta \theta_{\text {int }}=\frac{1}{2 \tau} K_{v} t^{2} \text { update }
$$


Walker discussed the phase step response in his work. However, the timing model used is not accurate, which led to an incorrect conclusion in which Walker stated that the magnitude of the oscillatory transient of a second order loop step response can be considered bounded by the simple linear response of the first order loop.

\subsection{Summary}

A review of architecture of both linear and bang-bang CDR with different alternative linear phase detector implementations were presented and compared in terms of their integration technology versus their jitter performance and static phase offset. The bangbang architecture was introduced with emphasis on how the bang-bang phase detector is utilized using the Alexander phase detector.

Definitions of jitter performance in high speed digital communication standards were extracted and defined, followed by a review of the literature of first order bang-bang loop and second order loop, which showed a lack of theory and analysis for the bang-bang CDR. 


\section{First Order Bang- Bang Clock and Data Recovery}

\subsection{Introduction}

The bang-bang CDR is conceptually simple. It is therefore all the more surprising to find that the system is difficult to analyze. The loop prohibits simple analysis because of the nonlinearity of the phase detector. In addition, the time sampler causes the output phase to be both time and amplitude quantized, which adds to the difficulty of analysis.

The objective of this chapter is to describe the behavior of the first order bangbang CDR and to discuss its important characteristics and limitations. Two important parameters in describing the performance of the loop, signal-to-noise ratio (section 3.6) and slope overload condition (section 3.4), are discussed. Techniques are used to evaluate the loop performance at given bit-error-rate when the input is a Gaussian jitter frequency.

In the last three sections, the loop jitter performance as a function of design loop parameters are discussed in detail. The models, equations and expressions developed in this chapter were either driven analytically and verified with time domain simulation. All graphs presented in this chapter are obtained from simulating a Matlab model of first order loop. 


\subsection{First-Order Loop Dynamics}

The first order loop dynamics can be explained and modeled as a linear Delta Modulator (DM) system. The first order bang-bang PLL loop can be considered a first order linear Delta Modulator as shown in Figure 3.1. The input phase $\theta_{d}\left(t_{n}\right)$ is a band limited signal over a band of frequencies $\left\{f_{c 2}, f_{c 1}\right\}$ where $f_{c 2}>f_{c 1}$. The output of the sampleand-hold block $\mathrm{q}(\mathrm{t})$ samples at rate $f_{n}$ where $f_{n}=1 / t_{n}$, where $f_{n}$ is considerably greater than the Nyquist rate $2 f_{c 2}$. In fact, the input jitter band is always bounded to half of the input

data stream rate $\frac{1}{t_{d}}$, and $f_{\mathrm{n}}$ is set to be $\frac{1}{2^{m} t_{d}}$ where $\mathrm{m}$ is $\{1,2,3,$.$\} . The relationship between$ input phase $\theta_{d}(t)$ and $\mathrm{q}(\mathrm{t})$ is such that $\mathrm{q}(\mathrm{t})$ is a binary representation to the instantaneous slope of $\theta_{d}(t)$. In other words, it is a binary representation to the instantaneous input frequency $f_{i n}$. If the slope of $\theta_{d}(t)\left(f_{i n}\right)$ is positive, then while this condition is valid the output of $\mathrm{q}(\mathrm{t})$ has more positive pulses than negative pulses, and the situation is reversed when the slope of $\theta_{d}(t)\left(f_{i n}\right)$ is negative.

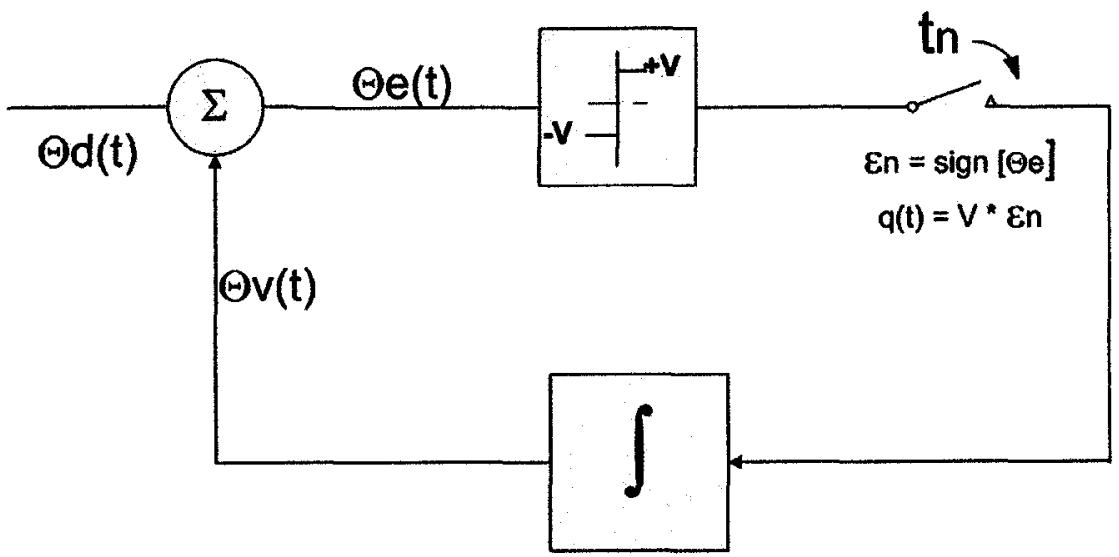

Figure 3.1 First order bang-bang PLL 
Figure 3.2 shows how the output phase of the first order loop varies with time when the input phase is a sinusoidal. All the waveforms shown are normalized. The binary pulse waveform $\mathrm{q}(\mathrm{t})$ is integrated by the integrator in the feedback loop. The resulting waveform $\theta_{v}(t)$ ramps with slope $V \mathrm{rad} / \mathrm{sec}$ and oscillates around the input phase $\theta_{d}(t)$. The difference between $\theta_{e}(t)=\theta_{d}(t)-\theta_{v}(t)$ is called phase error $\left(\theta_{\text {error }}\right)$ or quantization error. This phase error is quantized to the limits $\pm V$ (loop gain in $\mathrm{rad} / \mathrm{sec}$ ). Notice that the sign, not the magnitude, of the phase error is quantized. The output of the quantizer (limiter) is sampled every $t_{n}$ to produce $\mathrm{q}(\mathrm{t})$. The phase changes by $\theta_{b b}=V t_{n}$ radians (bangbang phase step) during every update time $\left(t_{n}\right)$.
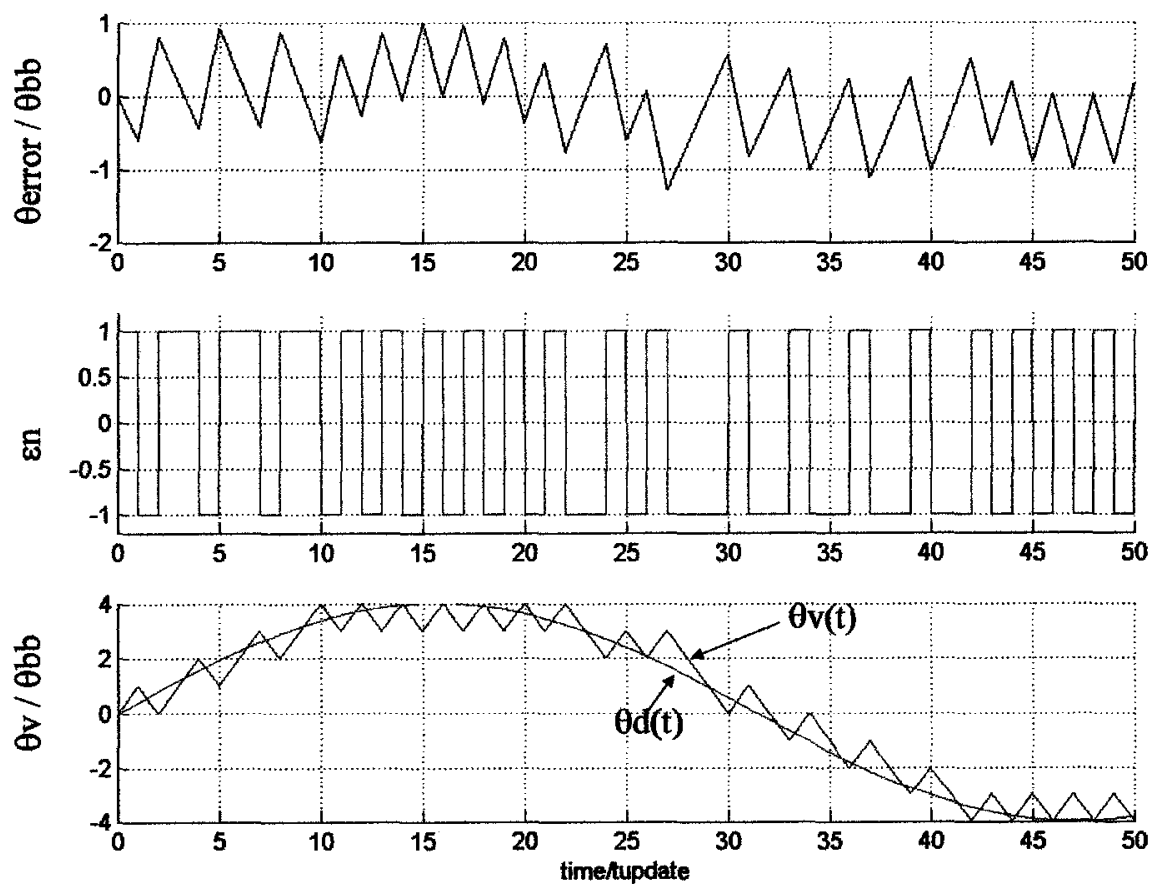

Figure 3.2 Simulated first order loop waveforms correctly tracking the input phase 
As long as $\theta_{e}(t)$ is positive, a sequence of positive binary pulses will be produced at each clock transition instant, which is integrated to increase the output phase $\theta_{v}(t)$. Eventually, $\theta_{v}(t)$ will become greater than $\theta_{d}(t)$, leading to $\theta_{e}(t)$ becoming negative at a clock transition instant. As a result, a negative binary pulse will be produced which decreases $\theta_{v}(t)$. The loop attempts to minimize the phase error by varying the polarity of binary pulses at the output of the limiter. The loop attempts to generate a $q(t)$ pattern with a mean value over short period of time approximately equal to the mean value of the slope of the input phase. In other words, the loop generates a DC voltage which sets the average VCO frequency to be equal to the average input frequency over a short period of time.

\subsection{Idling pattern}

When no $\theta_{d}(t)$ is applied at the input of the first order loop, the loop is considered to be in its idling state. In a steady state the binary waveform $q(t)$ has alternating positive and negative pulses resulting in a feedback $\theta_{v}(t)$ that has a sawtooth waveform with a period equal to $2 t_{n}$ and an amplitude of $\pm\left(V t_{n}\right)^{\prime 2}$. The idling pattern of the first order loop

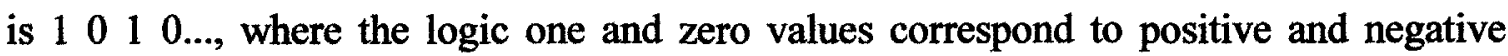
pulses of $q(t)$ respectively. The loop is called symmetrical if the slope of $\theta_{d}(t)$ required to produce all the "ones" in the pattern has the same magnitude and opposite polarity as the slope of $\theta_{d}(t)$ required to produce all the "zeros" in the pattern. Studying the asymmetrical first order loop is out of the scope of this thesis. 
The idling oscillatory pattern does not represent an instability situation of the first order loop. It must be highlighted that the first order bang-bang loop is very stable and the idling pattern of $\theta_{v}(t)$ has a bounded amplitude and fixed periodic waveform.

\subsection{Slope Overload}

When the loop is correctly tracking the input phase, the phase error is always bounded $\left|\theta_{e}(t)\right| \leq V t_{n}$, and the output phase $\theta_{v}(t)$ always varies around the input phase $\theta_{d}(t)$. However, when the slope of the input phase increases, the loop attempts to generate $q(t)$ with a sequence of similar polarity pulses to minimize the phase error. As shown in the simulated response in Figure 3.3, if the slope of the input phase increased to a value greater than the maximum slope of $\theta_{v}(t)$, then the loop will fail to track the input phase and the phase error will exceed $v t_{n}$. This condition, where the output phase does not track the input phase, is referred to as "slope overload". 

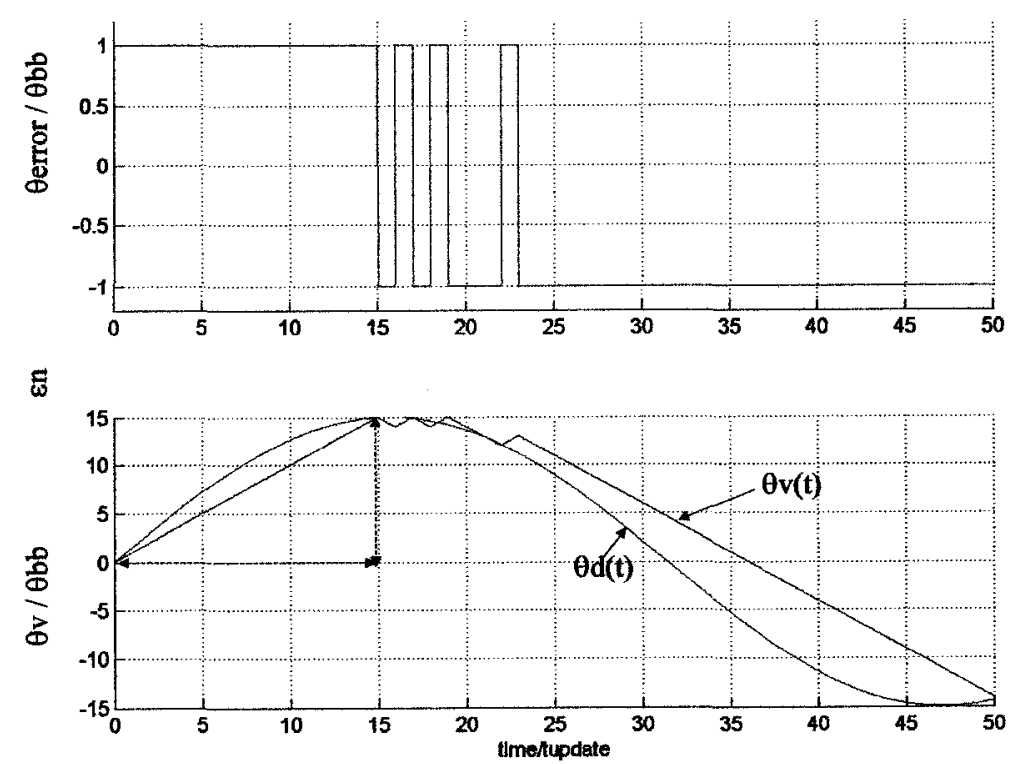

Figure 3.3 Simulated normalized output phase waveform when the first order loop is slope overloaded

\subsubsection{Slope Overload due to DJ}

Assuming the input jitter is a sinusoidal and evaluated at each update time $\left(t_{n}\right)$, then we get the following formulas:

$$
\begin{gathered}
\theta_{d}\left(t_{n}\right)=m_{j} \sin \left(2 \pi f_{j} t_{n}+\Phi_{i}\right) \\
\theta_{d}^{\prime}\left(t_{n}\right)=m_{j} \cdot 2 \pi f_{j} \cdot \cos \left(2 \pi f_{j} t_{n}+\Phi_{i}\right)
\end{gathered}
$$

where $m_{j}$ is the input sinusoidal jitter amplitude.

From section 3.2, the maximum rate of increase of $\theta_{v}(t)$ is given by

$$
\frac{d}{d t} \theta_{v}\left(t_{n}\right)=V
$$


where $V$ is the loop gain in $\mathrm{rad} / \mathrm{sec}$.

The condition of the slope overload is generally avoided if $\theta_{d}^{\prime}\left(t_{n}\right) \leq \frac{d}{d t} \theta_{v}\left(t_{n}\right)$, and therefore

$$
m_{j} \cdot 2 \pi f_{j} \leq V
$$

The first order loop can not track high frequency jitter without invoking a slope overload unless the jitter amplitude is restricted and/or the loop gain, $V$, is increased. The loop gain (and consequently $\theta_{b b}$ ) is the only circuit parameter to be adjusted in order for the loop to correctly track the input phase. To avoid an overload in the loop, the minimum loop gain $V_{\min }$ to a sinusoidal input is then given by

$$
V_{\min }=\left.\left(m_{j} \cdot \omega_{j}\right)\right|_{\max }
$$

where $\omega_{j}$ is the angular jitter frequency.

In SONET standards, the maximum value of $\left(m_{j} \cdot \omega_{j}\right)$ occurs at maximum jitter frequency defined by the jitter tolerance mask.

\subsubsection{Slope Overload due to RJ}

As described in chapter 2 , the input jitter has an unbounded low probability random jitter component $(R J)$. The distribution of the $R J$ is defined as Gaussian. In order to develop the probability of invoking a slope overload, we assume the input jitter, $\theta_{d}$, is a Gaussian signal, band limited from 0 to $f j b \mathrm{~Hz}$, and $\sigma^{2}$ is the mean square value of the $\theta_{d}$. 
The condition to avoid the slope overload is generally given by

$$
\theta_{d}^{\prime} \leq \frac{d \theta_{v}}{d t}=V
$$

where $\theta_{d}^{\prime}=\frac{d \theta_{d}}{d t}$

There is always a probability that the loop will be slope overloaded if the input jitter is band limited white noise. However, to make this probability insignificant, the r.m.s. value of the input jitter frequency, $\theta_{d}^{\prime}$, has to be sufficiently smaller than the maximum slope of $\theta_{v}, V$. The mean square value of $\theta_{d}^{\prime}$ is $\left(\sigma_{d}{ }^{2}\right)$ which is defined as

$$
\begin{gathered}
\sigma_{d}^{2}=\int_{-f j b}^{j b}(2 \pi f)^{2}\left(\frac{\sigma^{2}}{2 f j b}\right) d f \\
\sigma_{d}^{2}=\left(\frac{2 \pi^{2} \sigma^{2}}{f_{j b}}\right) \cdot \int_{-f j b}^{j b}(2 \pi f)^{2} d f
\end{gathered}
$$

where $\left(\frac{\sigma^{2}}{2 f j b}\right)$ is the spectral density function of band limited white noise, and $\left(2 \pi f^{2}\right.$ is the power transfer function of first order differentiation. Therefore,

$$
\sigma_{d}^{2}=\frac{4}{3} \pi^{2} f_{j b}^{2} \sigma^{2}
$$


The probability of error can be expressed as

$$
B E R=\operatorname{erfc}\left(\frac{a}{\sqrt{2} \cdot \sigma_{d}}\right)
$$

where $e r f c$ is the complementary error function and defined as

$$
\operatorname{erfc}(x)=\frac{1}{\sqrt{2 \pi}} \int_{x}^{\infty} e^{-\frac{y}{2}} d y
$$

and $a$ is the decision threshold, expressed in multiples of $\sigma_{d}$

$$
a=C \sigma_{d}
$$

We call $C$ the confidence level. Equation 3.10 can therefore be expressed as

$$
B E R=\operatorname{erfc}\left(\frac{C}{\sqrt{2}}\right)
$$

The general condition of the loop to the Gaussian input jitter in order to experience slope overload with a probability less than BER is given by

$$
C \cdot \sigma_{d} \leq \frac{d \theta_{v}}{d t}
$$


Substituting the value of $\sigma_{d}$ and $\frac{d \theta}{d t}$ from Equation 3.9 and 3.6 respectively, we get:

$$
\sigma^{2} \leq \frac{3}{4 \pi^{2}} \cdot \frac{1}{f_{j b}^{2}} \cdot\left(\frac{V}{C}\right)^{2}
$$

Therefore,

$$
\sigma_{\max }^{2}=\frac{3}{4 \pi^{2}} \cdot \frac{1}{f_{j b}^{2}} \cdot\left(\frac{\eta}{C}\right)^{2}
$$

For the probability of the loop to experience a slope overload less than $10^{-10}, c^{2}=41.82$, then the slope overload condition is

$$
\sigma^{2} \leq \frac{3}{4 \pi^{2}} \cdot \frac{1}{f_{j b}^{2}} \cdot\left(\frac{V}{41.82}\right)^{2}
$$

The minimum loop gain $V_{\min }$ required for the RJ input to have better than $\mathrm{BER}=10^{-10}$ is

$$
V_{\min }=\left.\frac{13 \pi}{\sqrt{3}}\left(\sigma f_{j b}\right)\right|_{\max }
$$

where $f_{j b}$ is the maximum random jitter frequency component, or the limiting filler (Golden PLL) corner frequency which is defined by standards (e.g. for unfiltered jitter $\left.f_{j b}=0.5 \times f_{n}\right)$ 


\subsection{Amplitude Dynamics}

The maximum amplitude of sinusoidal input jitter $m_{j m a x}$ that can be tracked correctly without overloading the loop is given from inequality 3.4 .

$$
m_{j \max }=\frac{V}{2 \pi f}
$$

where $m_{j \max }$ is the maximum input sinusoidal jitter amplitude.

When the loop is in its idling state, the output phase $\theta_{v}(t)$ is a periodic sawtooth waveform with a peak-to-peak amplitude $V / f n$. In order to disturb this idling pattern, the sinusoidal input jitter must have an amplitude greater than $V / 2 f n$. The loop is incapable of tracking a sinusoidal input jitter with $m_{j} \geq\left(\frac{V}{2 f_{n}}\right)$. When $m_{j}$ starts to become greater than $V / 2 f n$, the loop starts to track the input jitter. The ratio of $m_{j \max }$ to the value of $m_{j}$, which is just disturbing the idling pattern, is defined as jitter amplitude dynamic range (JADR). Hence,

$$
J A D R=\frac{f_{n}}{\pi f_{j}}
$$

\subsection{Quantization Noise}

The quantization noise is defined as the loop phase error, $\theta_{e}(t)$ within the loop bandwidth while the loop is correctly tracking. However, outside of the loop bandwidth, the slope overload noise becomes dominant. While the quantization process is a non-linear process, a linear model for the quantizer can be employed whenever the loop is not slope 
overloaded as show in Figure 3.4 [43]. The quantization error $\mathrm{e}(\mathrm{n})$ is not then considered correlated with input phase changes and is bounded by $\pm V /\left(2 f_{n}\right)$, and has a white spectrum and equal probability over the range $\pm V /\left(2 f_{n}\right)$.

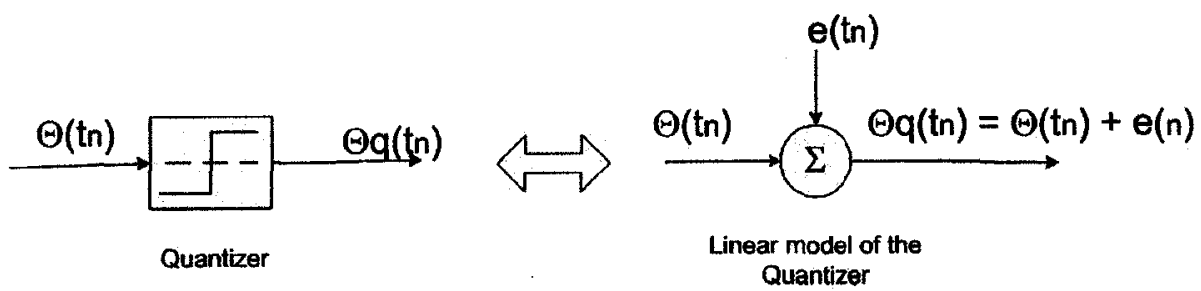

Figure 3.4 The Linear Model of the Quantizer

We assume that the input jitter is random or periodic, and not correlated to the clock signal. Further, we assume that $S_{n n}(f)$, the spectral density of $\theta_{e}(t)$, is flat over the jitter bandwidth. With analogy to linear Delta Modulation, the quantization noise, $N_{q}{ }^{2}$, is proportional to the jitter frequency band $f_{j b}$ and as the clock rate $f_{n}$ is increased, quantization error is improved linearly with $f n$. Hence, $N_{q}{ }^{2} \propto 1 / f_{n}$. In addition, increasing the bangbang phase $\theta_{b b}$ increases the amplitude of $\theta_{e}(t)$ and consequently $N_{q}{ }^{2} \propto \theta_{b b}^{2}$. Hence,

$$
N_{q}^{2}=K_{q} \frac{f_{j b}}{f_{n}} \theta_{b b}^{2}
$$

where $K_{q}$ is a empirical constant and $0.3<K_{q}<0.9$ [44]. 
The signal-to-quantization noise ratio (SQNR) is defined as the ratio of input jitter power $s^{2}$ to the quantization noise $N_{q}^{2}$ whenever the loop is tracking and not slope overloaded. The signal-to-quantization noise is an important parameter to determine the quality of tracking.

\subsubsection{SQNR of DJ}

For sinusoidal input jitter with an amplitude of $m j$, the input power is $m_{j}^{2} / 2$ and the SQNR is therefore

$$
S Q N R=\frac{1}{2 K_{q}} \frac{f_{n}}{f_{j b}}\left(\frac{m_{j}}{\theta_{b b}}\right)^{2}
$$

Since the quantization noise power $N_{q}{ }^{2}$ is independent of jitter power $s^{2}$, then SQNR is linearly proportional with $s^{2}$ and has a maximum value when $\left.s^{2}\right|_{\max }=\left(m_{j \max }\right)^{2 / 2}$

From Equations 3.19 and 3.22, we get:

$$
S Q N R_{m a x}=\frac{1}{8 \pi^{2} K_{q}} \cdot \frac{f_{n}^{3}}{f_{j b} \cdot f_{j}^{2}}
$$

When $m j$ exceeds mjmax, the loop experiences a slope overload and the quantization noise increases with a rate greater than the rate of increase of $s^{2}$, resulting in a decrease in SQNR. 


\subsubsection{SQNR of $R J$}

For Gaussian input jitter with variance $\sigma$, the input power is $\sigma^{2}$ and the SQNR is therefore given by

$$
S Q N R=\frac{1}{K_{q}} \frac{f_{n}}{f_{j b}}\left(\frac{\sigma}{\theta_{b b}}\right)^{2}
$$

From Equations 3.16 and 3.24, the maximum SQNR can be expressed as

$$
S Q N R_{\max }=\frac{1}{4 \pi^{2} K_{q} C^{2}} \cdot\left(\frac{f_{n}}{f_{j b}}\right)^{3}
$$

\subsection{Model of First order-Loop}

The first order loop can be modeled as a first order linear Delta Modulation system as shown in Figure 3.5 where the summing node and the quantizer represent the bangbang phase detector and $\beta$ is a scaling factor in volts to attenuate the signal controlling the VCO. The VCO has a gain of $K_{v}$ in $\mathrm{rad} / \mathrm{sec} / \mathrm{volt}$. $D F$, the density factor, is the average transition density of the incoming data stream. For example, a fully random data stream has $D F=0.5$ and for data transition alternating with pattern $10101 \ldots, D F=1$.

The model assumes the loop has uniform sampling times, $t_{n}$, which is a reasonably accurate assumption for our application since the instance change in frequency (i.e. the bang-bang frequency $f b b$ ) is usually in the order of $0.1 \%$ of the normal VCO frequency $f o$. The model assumes that the phase detector is a ternary phase detector with hold state. The ternary phase detector is widely used for its superior jitter performance for data with long runs [38].

The input phase, $\theta d$, is considered to be generated from a pattern generator clocked by a clock signal $\sin \left(\left(\omega_{n}+\delta \omega\right) \cdot t+\phi\right)$, and then the input phase is defined as 
$\theta_{d}\left(t_{n}\right)=\delta \omega \cdot t_{n}+\phi\left(t_{n}\right)$ at $\mathrm{n}^{\text {th }}$ sampling time $t n$. The input phase is composed of two components; a frequency variation component $\delta \omega \cdot t_{n}$ and phase variation component $\phi\left(t_{n}\right)$.

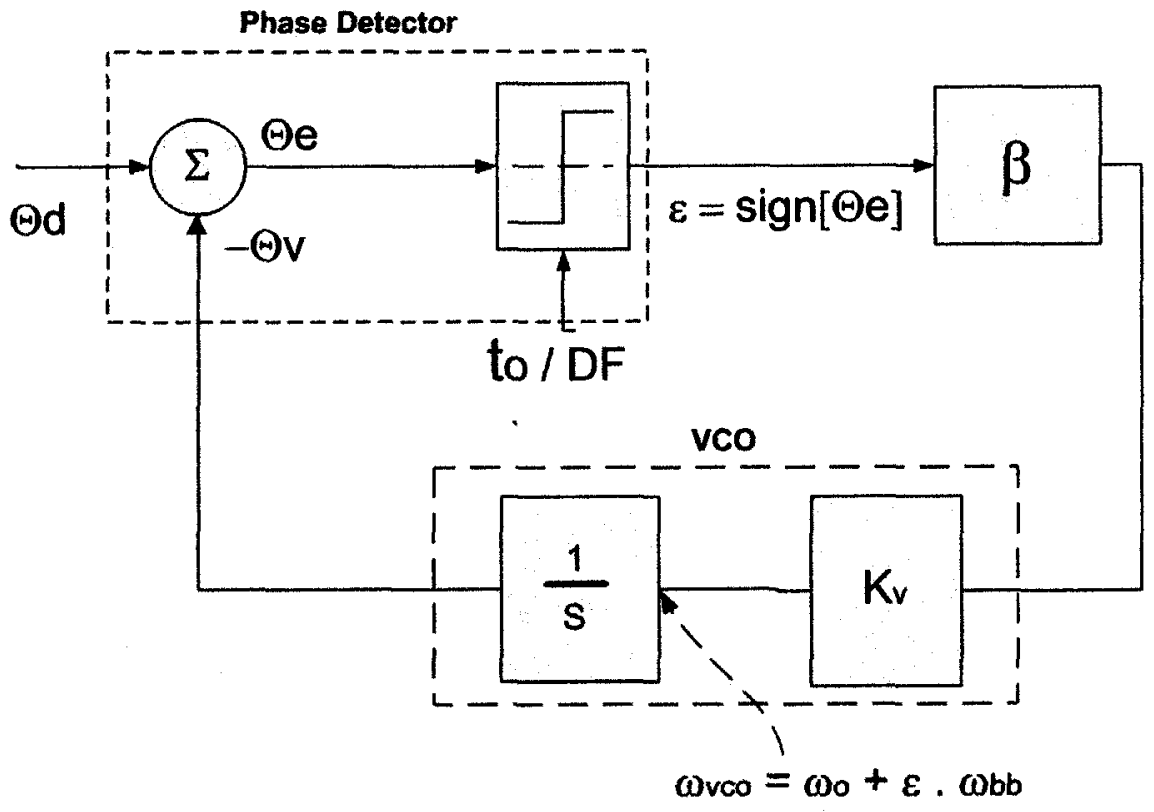

Figure 3.5 First order bang-bang loop model based on linear Delta Modulation

The general first order bang-bang model is now defined such that

$$
V=\beta \cdot K_{v}=\omega_{b b} \quad(\mathrm{rad} / \mathrm{sec})
$$

and

$$
t_{n}=\frac{t_{o}}{D F}
$$

where $\omega_{b b}$ is the bang-bang angular frequency in $\mathrm{rad} / \mathrm{sec}$. 
The set of discrete time difference equations describing the loop dynamics is defined by Equations $2.11,2.12$ and 2.13 where

$$
\theta_{b b}=\omega_{b b} \cdot t_{n}=2 \pi\left(\frac{f_{b b}}{D F \cdot f_{o}}\right)
$$

Another useful rearrangement of the general model is represented in Figure 2.12 when the input phase is due to a frequency variation component $\delta \omega \cdot t_{n}$ and $\phi\left(t_{n}\right)=0$. The rearranged model as shown in Figure 3.6 is a Delta-Sigma Modulation system. The DeltaSigma Modulation system is an equivalent model to the linear Delta Modulation model with preceding integrator. In this report both models are going to be used to drive the analysis of the first order bang-bang loop.

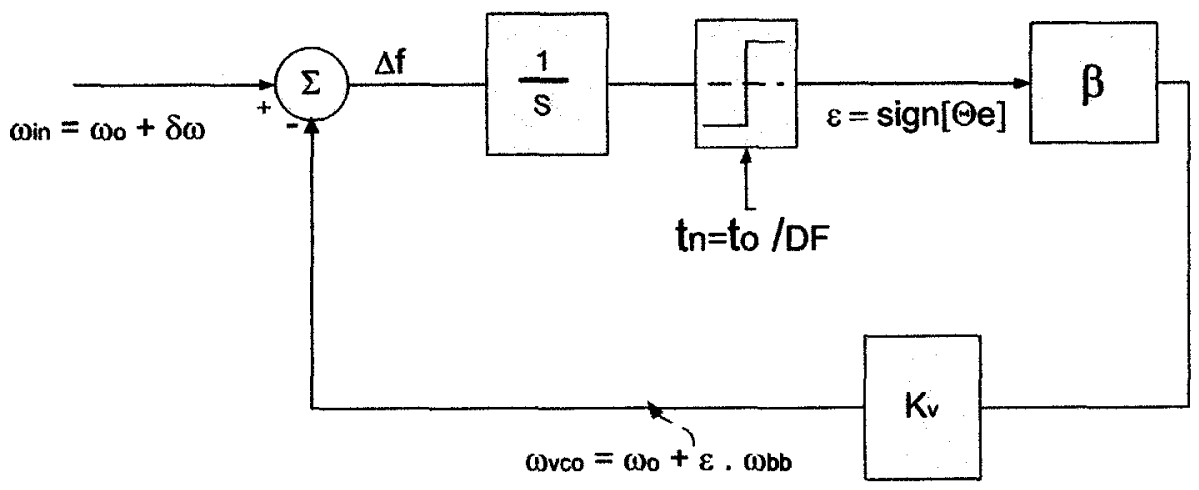

Figure 3.6 First order bang-bang loop model based on Delta Sigma Modulation

\subsection{First order bang-bang linearized model}

The noise performance of the first order bang-bang loop can be examined by employing widely used methods in analysis of oversampling converters. In this method, a 
linear model of the quantizer is used as discussed in section 3.6. Thus, the first order loop based on linear Delta Modulation can be drawn using a linear model for the quantizer as shown in Figure 3.7. $S_{h}$ is the transfer function of the sample and hold circuit and is defined as [43]

$$
\left.S_{h}(s)\right|_{s \rightarrow \omega}=\frac{2 \pi}{\omega_{n}} \cdot \frac{\sin \left(\pi \frac{\omega}{\omega_{n}}\right)}{\left(\pi \frac{\omega}{\omega_{n}}\right)}
$$

where $\mathrm{H}(\mathbf{s})$ is defined as

$$
\left.H(s)\right|_{s \rightarrow \omega}=S_{h} \beta K_{v}
$$

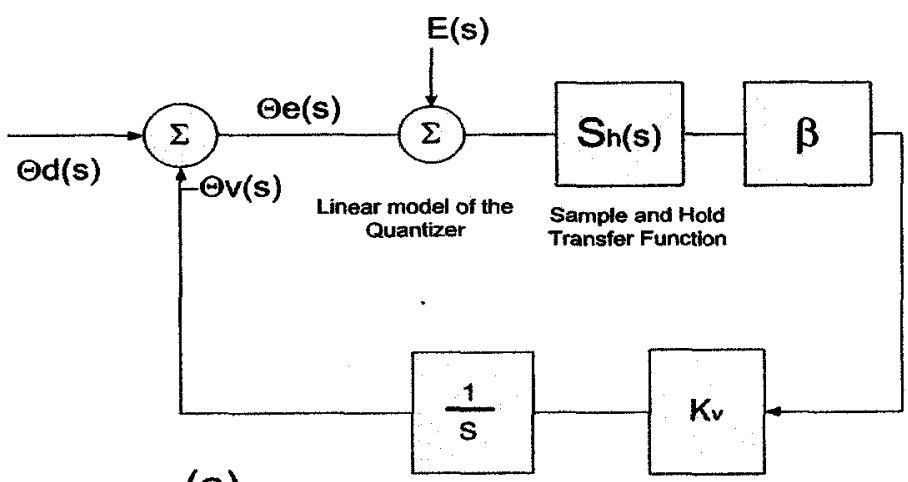

(a)

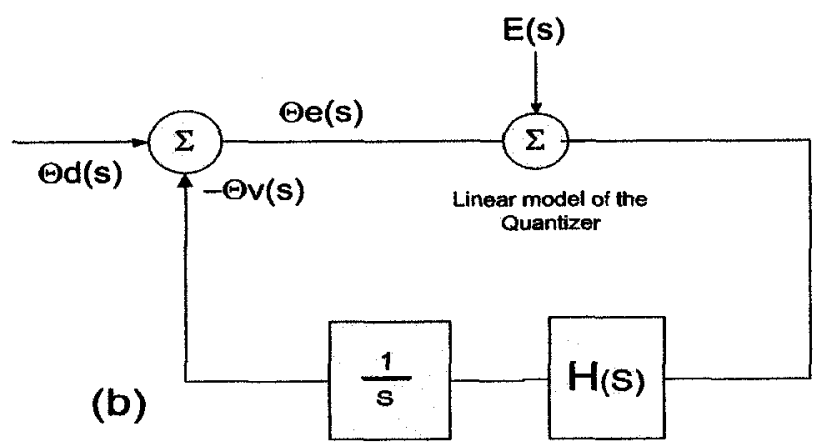

Figure 3.7 Mathematical mode for analyzing first order loop employing a linear model for quantizer; (a) actual model (b) simplified model 
The analysis of the above loop can be expressed as

$$
\theta_{v}(s)=\frac{H(s)}{s+H(s)} \cdot \theta_{d}+\frac{H(s)}{s+H(s)} \cdot E(s)
$$

The output phase $\theta_{v}(s)$ can be described by means of two transfer functions; phase transfer function (PTR) and noise transfer (NTR). It is given by

$$
\theta_{v}(s)=P T R \cdot \theta_{d}+N T R \cdot E(s)
$$

where

$$
P T R=N T R=\frac{H(s)}{1+H(s)}
$$

The output phase is a sum of the input phase and the quantization noise within the loop bandwidth. The quantization noise lying outside the loop bandwidth is rejected by the low pass filter.

Similarly, the Delta Sigma Model can be linearized as shown in Figure 3.8. 


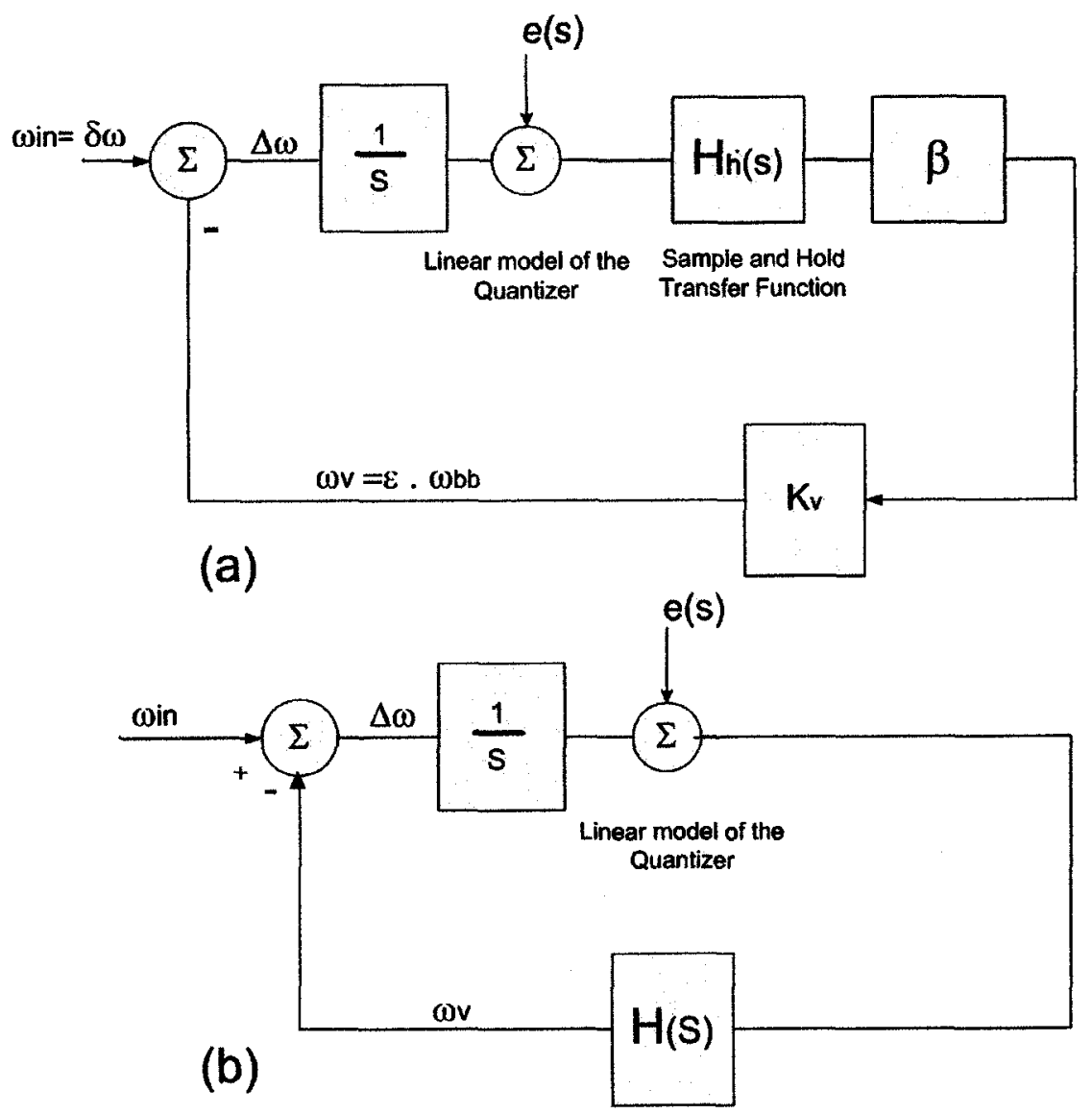

Figure 3.8 Mathematical model for analyzing first order loop employing a linear model for quantizer; (a) actual model (b) simplified model

The transfer function of the loop can be written as

$$
\omega_{v}(s)=\frac{H(s)}{s+H(s)} \cdot \omega_{i n}+\frac{H(s) \cdot s}{s+H(s)} \cdot E(s)
$$

The quantization noise has a minimum effect on output frequency as a result of quantization noise shaping over the loop bandwidth, which is filtered out by the high pass filter. 


\subsection{Phase Step Response}

In order to get the phase step response of the first order bang-bang loop, the loop is assumed to be in lock, $\delta \omega=0$ and $\phi=\operatorname{\theta r}(t)$, where $(t)$ is the unit step function at $t=0$ as shown in Figure 3.9 and $\theta$ is the size of the phase step. A Matlab model follows the diagram shown in Figure 3.5 is utilized to simulate the loop response and validate the equations.

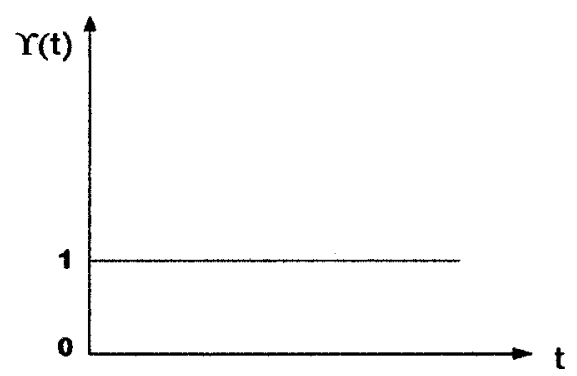

\section{Figure 3.9 Unit step function $Y(t)$}

The maximum slope input phase at $t=0$ is infinite and exceeds the maximum slope of $\theta_{v}(t)$. Therefore the loop will fail to track the input phase and will be slope overloaded, consequently stopping toggling and leading to a transient phase error. The output phase increases each update time, $t m$, with its maximum rate until the phase error flips its sign. From Equations 3.3, 3.26, and 3.28 the "lock-in-phase" time required by the loop to start tracking the incoming phase is

$$
t=\frac{\theta}{\beta K_{v}}
$$

Then

$$
n=\frac{t}{t_{n}}=\frac{\theta}{\theta_{b b}}
$$


where $n$ is number of cycles of $t n$.

As shown in Figure 3.10, the simulation of normalized phase error shows an agreement to Equation 3.36. The loop is simulated with different phase steps and shows lockin-phase time required in order for the phase error to vanish.

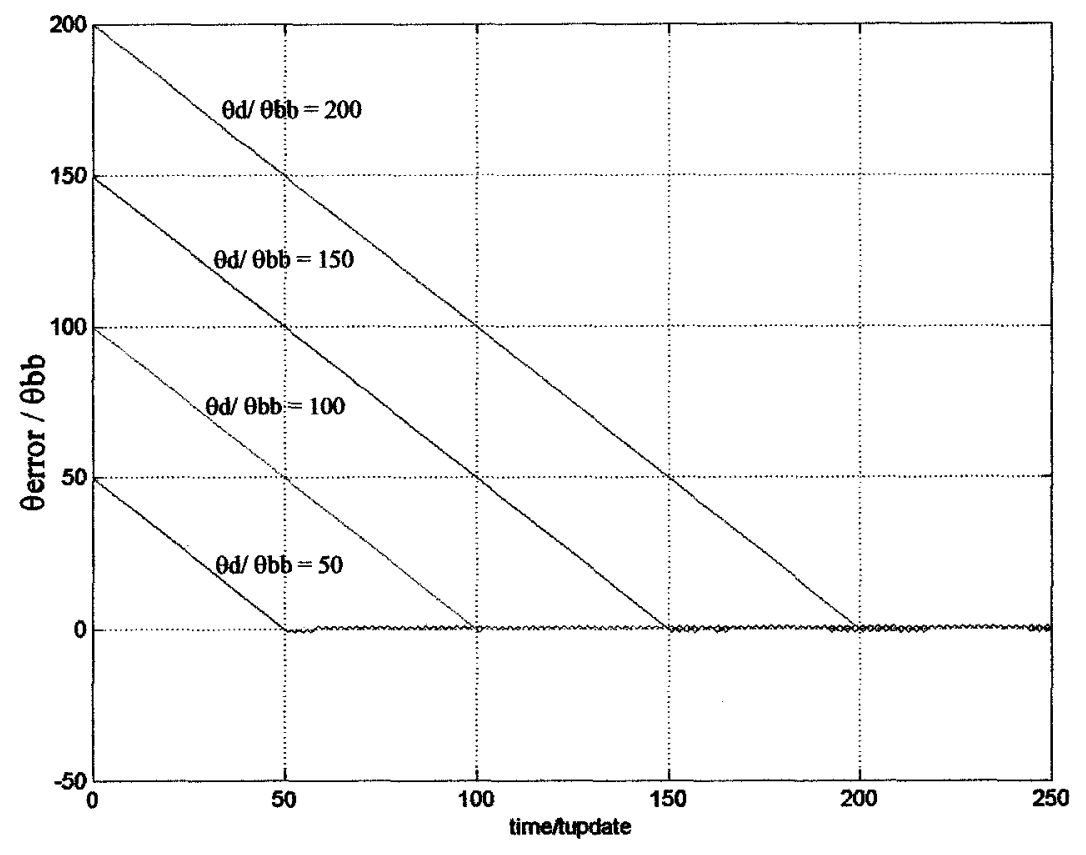

Figure 3.10 Simulated response of first order loop to range of input steps

The effect of changing $\theta_{b b}$ is shown in Figure 3.11. Increasing $\theta_{b b}$ increases the maximum slope of $\theta_{v}(t)$ and hence reduces the lock-in-phase time, but the loop will experience larger jitter generation as will be discussed in Section 3.12. As expected, the loop response does not experience any overshoot to a phase step at the input. 


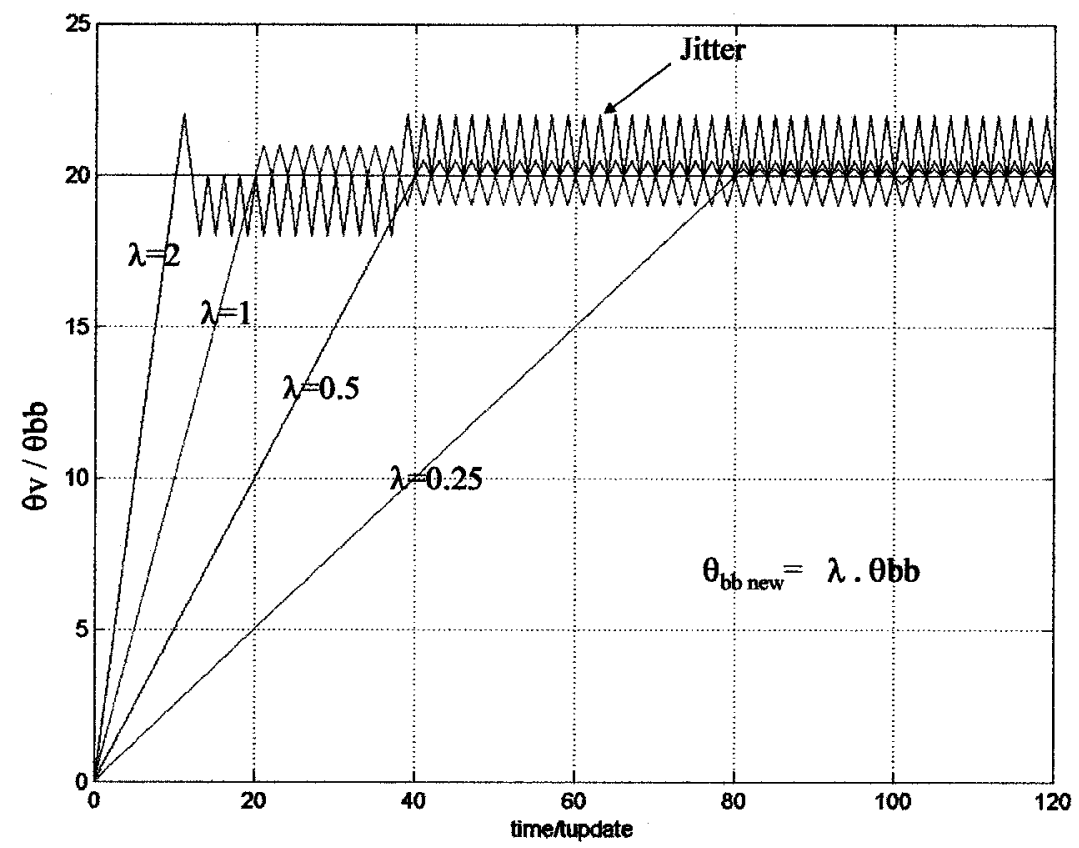

Figure 3.11 Simulated the first order loop response to range of bang-bang phase steps when the input is a step in time

\subsection{Frequency Step Response}

In order to get the frequency step response of the first order bang-bang loop, the loop is assumed to be $\theta_{d}=\delta \omega t \Upsilon(t)$ and $\phi=0$ wherer(t) is the unit step function at $t=0$ as described in the previous section. A Matlab model follows the diagram shown in Figure 3.6 is utilized to simulate the loop response and validate the equations. From Equations 3.3 and 3.26 the condition to avoid the slope overload is

$$
\delta \omega \leq\left|\omega_{b b}\right|
$$

Thus,

$$
-\omega_{b b} \leq \delta \omega \leq \omega_{b b}
$$


As long as the input frequency step is bounded by a bang-bang frequency step, the loop will remain phase locked with zero steady state phase error. A DC voltage proportional to $\delta \omega$ is built at the input of the VCO which pushes its center frequency to be equal to the incoming data frequency as shown in Figure 3.12. If $\delta \omega$ falls behind $\omega_{b b}$, the loop goes out of lock and whenever $\delta \omega$ is within the range the loop regains the lock.

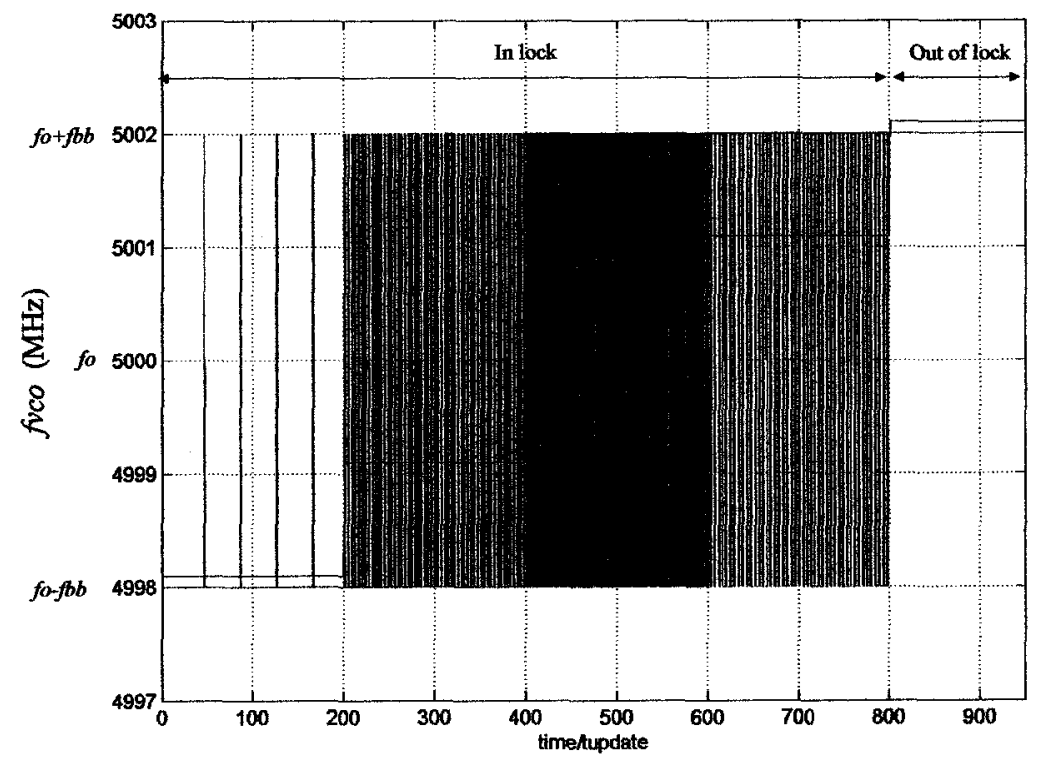

Figure 3.12 Simulated response of the first order loop to a range of input frequency steps

The first order loop has a narrow lock range $\left\{-f_{b b},+f_{b b}\right\}$. Increasing $f_{b b}$ will increase the lock range but degrades the jitter performance.

\subsection{Sinusoidal Operating Conditions}

The sinusoidal operating conditions are important to determine certain jitter performances. The analysis of the sinusoidal operating conditions assumes that the loop is 
phase locked. As well, it is assumed that there is no frequency offset between the incoming frequency and the VCO. In other words, $\delta \omega=0$ and $\phi=m_{j} \sin \left(\omega j \cdot t+\varphi_{o}\right)$ where $m_{j}$ is the input phase (jitter) amplitude in $\mathrm{rad}, \omega_{j}$ is the jitter angular frequency in $\mathrm{rad} / \mathrm{sec}$, and $\varphi_{o}$ is a zero mean phase shift. A Matlab model follows the diagram shown in Figure 3.5 is utilized to simulate the loop response and validate the equations. As given by Equations 3.19 and 3.26, the maximum jitter amplitude that can be tracked without slope overload is

$$
m_{j \max }=\frac{\omega_{b b}}{\omega_{j}}
$$

Furthermore, the maximum input frequency (the corner frequency) where the loop can track the input jitter can be expressed as

$$
\omega_{j \max }=\frac{\omega_{b b}}{m_{j}}
$$

Figure 3.13 (simulation) shows that the loop behaves like a single-pole response with corner frequency that is equal to $\omega_{j m a x}$ and the characteristic falls with almost $20 \mathrm{~dB}$ per decade. The cut-off (corner) frequency is inversely proportional to the jitter amplitude as illustrated in simulated waveforms which is in agreement with Equation 3.40. 


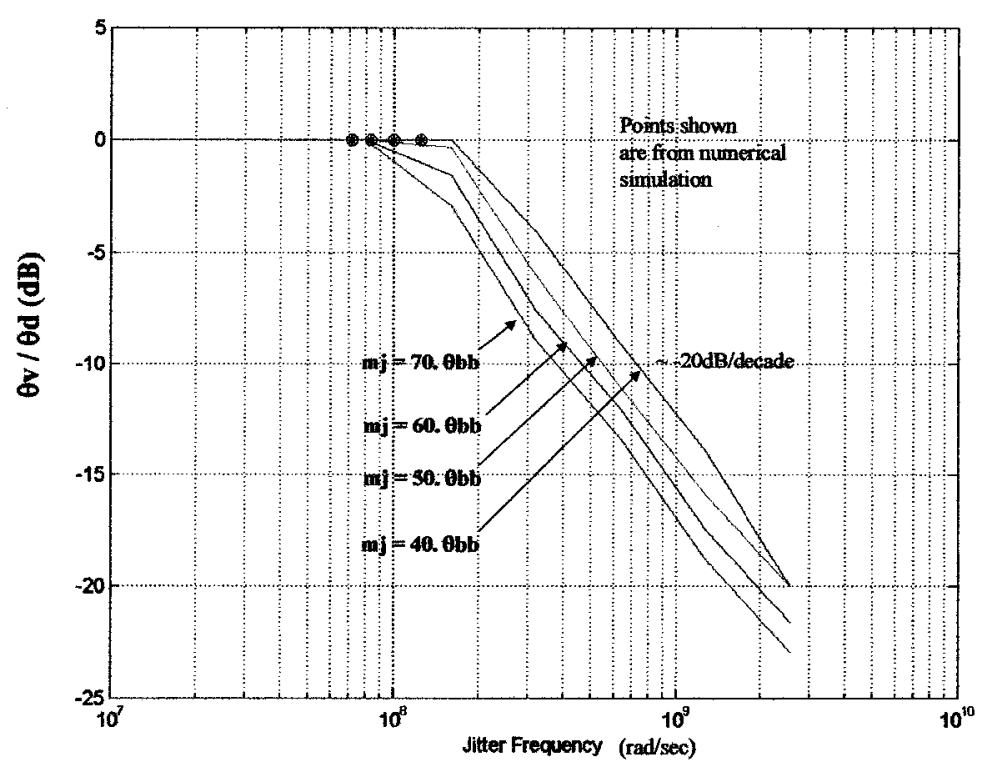

Figure 3.13 Simulated response of first order loop to a sinusoidal jitter with range of amplitudes

With regard to the phase error, the loop acts as a single pole high pass filter with cut of frequency equal to $\omega_{j \max }$. The loop has to have high $\omega_{b b}$ to reduce transient phase error.

\subsection{Jitter Analysis of First-Order Loop}

As discussed in Chapter 2, jitter is comprised of a deterministic jitter component $(D J)$ and a random jitter component $(R J)$. In order to analyze the jitter performance for the first order loop, certain assumptions will be made: First, the loop has no frequency offset between the incoming data frequency and VCO frequency. Second, the loop is assumed to be phase locked and does not experience a slope overload for jitter tolerance analysis. This assumption enables us to use the linearization model of first order loop. A Matlab model follows the diagram shown in Figure 3.5 is utilized to simulate the jitter performance and validate the equations. 


\subsubsection{Jitter Transfer}

As defined in the previous chapter, jitter transfer is the ratio of output jitter amplitude to the sinusoidal input jitter amplitude. Jitter transfer measurement [21] and simulation shows that the jitter transfer is considered as a single pole low pass filter with a corner frequency given by Equation 3.40. The calculated corner frequency is at $0 \mathrm{~dB}$. However, the jitter transfer function can be approximated to a curve that has a single pole of $\omega_{j \max }$ at $-3 \mathrm{~dB}$. This is similar to the SONET standard which approximates the jitter corner frequency to $-3 \mathrm{~dB}$. This makes the comparison of our approximation to that of a standard a fair comparison, in turn, between the two original curves.

Hence the jitter transfer function (JTR) is defined as

$$
J T R=\frac{1}{1+s / \omega_{\text {max }}}
$$

Then the bandwidth of the loop is

$$
B W=\frac{\omega_{b b}}{m_{j}}=\frac{\beta K_{v}}{m_{j} \cdot f_{o}}
$$

where $D F=1$ by definition from the standard as discussed in chapter 2 , where the jitter transfer test is performed using $101010 \ldots$ pattern.

Figure 3.14 shows that the simulated transfer function of the first order loop has an agreement with the Equation 3.40. The loop might experience a jitter peaking due to the quantization noise if it has low input jitter amplitude or high $\omega_{b b}$. The jitter peaking was discussed in the form of signal-to-quantization noise ratio (SQNR) by Equation 3.22. The jitter peaking $(\mathrm{JP})$ in $\mathrm{dB}$ is defined as 


$$
J P=10 \cdot \log \left(\frac{1}{S Q N R}\right)
$$

Therefore,

$$
J P=10 \cdot \log \left(\left(2 K_{q}\right) \cdot \frac{f_{j b}}{f_{n}} \cdot\left(\frac{\theta_{b b}}{m_{j}}\right)^{2}\right)
$$

which can be reduced to

$$
J P=10 \cdot \log \left(2 K_{q}\right)+10 \cdot \log \left(\frac{f_{i b}}{f_{n}}\right)-20 \cdot \log \left(\frac{m_{j}}{\theta_{b b}}\right)
$$

$\mathrm{Kq}$ is an empirical constant. The value of $K q$ for sinusoidal input is approximately $\frac{1}{3}$ [44].

The maximum jitter peaking of the first order loop occurs when $f_{j b}=0.5 \cdot f_{n}$ and the above equation becomes

$$
J P_{\text {max }}=-4.77-20 \cdot \log \left(\frac{m_{j}}{\theta_{b b}}\right)
$$

According to the standards, jitter peaking has to be less than $0.1 \mathrm{~dB}$ for all ranges of frequency. Thus the condition to satisfy the jitter peaking can be expressed as

$$
m_{j} \geq 0.57 \cdot \theta_{b b}
$$

Consequently, the required value $\theta_{b b}$ to meet jitter peaking is

$$
\left.\theta_{b b}\right|_{J P} \leq \frac{m_{j \min }}{0.57}
$$


where $m_{j m i n}$ is the minimum input jitter amplitude defined by the standards.

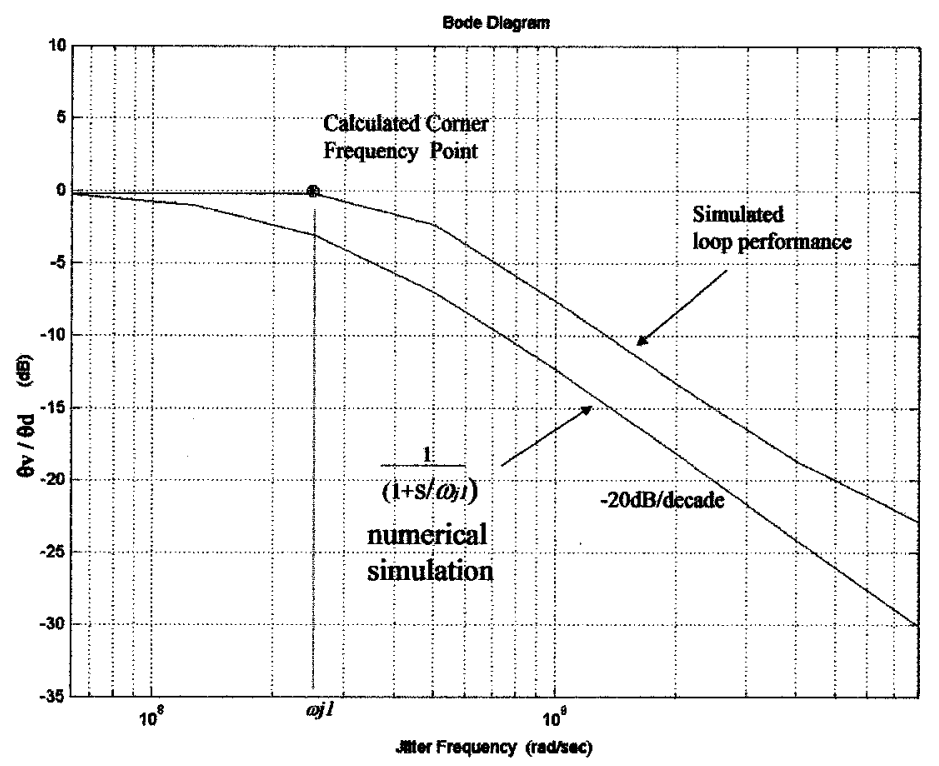

Figure 3.14 Simulated jitter transfer function of first order loop

\subsubsection{Jitter Tolerance}

As defined in Chapter 2, the jitter tolerance test is performed by applying the well controlled sinusoidal jitter at the clock and recording $B E R=10^{-10}$ hits. Degradation of the BER depends on the amount of deterministic and random jitters of the incoming data stream and the amplitude of the sinusoidal jitter of the clock. If the incoming data stream has less random jitter, then the loop can tolerate more sinusoidal jitter amplitude. On the other hand, if the incoming data has high random jitter then the loop tolerates less sinusoidal jitter amplitude. Therefore, in order to achieve the jitter tolerance $B E R=10^{-10}$, the condition of slope overload over jitter frequency band has to be developed and the incoming random jitter has to be bounded at $B E R$ better than $B E R=10^{-10}$. 
In order to establish the slope overload condition over jitter frequency band, the linear model of the first order loop can be applied. As a result of having sample frequency greater than jitter frequency, the effect of the hold circuit can be practically ignored. Applying the linear Delta Sigma model, the loop has the transfer function

$$
\delta \omega \approx \frac{s}{\omega_{b b}+s} \cdot \omega_{i n}
$$

Substituting

$$
\omega_{i n}(s)=s \cdot m_{j m a x}
$$

where

$$
\delta \omega=\omega_{b b}
$$

then

$$
m_{j \max } \approx \frac{\omega_{b b}}{s}+\left(\frac{\omega_{b b}}{s}\right)^{2}
$$

Equation 3.52 is an approximated formula given that the loop is linearized and that the quantization noise and hold circuit transfer function are not considered. However, the accurate jitter tolerance spectrum can be developed using the slope overload definition. Equation 3.39 precisely describes the slope overload condition over the frequency band. Replacing $\omega_{j}$ by 's'

$$
m_{j \max }=\frac{\omega_{b b}}{s}
$$


The maximum tolerated sinusoidal input jitter has a characteristic of a single integrator which falls off $20 \mathrm{~dB}$ per decade. The loop is more suitable to tolerate low frequency jitter than high frequency. Therefore the bang-bang angular frequency $\left.\omega_{b b}\right|_{J T L}$ required to meet deterministic jitter tolerance is

$$
\left.\omega_{b b}\right|_{J T L} \geq\left. m_{j \max }\right|_{\omega j \max } \cdot \omega_{j \max }
$$

where $\left.m_{j \max }\right|_{\Phi j \max }$ is the maximum jitter amplitude at the maximum jitter frequency defined by the jitter tolerance mask.

Similarly, $\left.{ }^{\omega_{b b}}\right|_{J T L}$ could be obtained from Equation 3.52 by solving for $\omega_{b b}$. Therefore,

$$
\omega_{b b}=\frac{-s+\sqrt{s^{2}+4 s^{2} m_{j \max }}}{2}
$$

which when simplified becomes

$$
\omega_{b b}=\frac{s}{2} \cdot\left(-1+\sqrt{1+4 m_{j \max }}\right)
$$

And since at high frequencies $m_{j m a x} \ll 1$, therefore

$$
\omega_{b b} \approx \frac{s}{2} \cdot\left(-1+\left(1+\frac{1}{2} \cdot 4 m_{j m a x}\right)\right)
$$

which reduces to

$$
\omega_{b b} \approx s \cdot m_{j m a x}
$$


Equation 3.54 sets the condition of meeting the deterministic jitter tolerance. However, the random jitter has to be bounded at bit error rate better than $B E R=10^{-10}$ by Equation 3.18 , which is repeated for convenience.

$$
\omega_{b b} \geq\left.\frac{13 \pi}{\sqrt{3}}\left(\sigma f_{j b}\right)\right|_{\max }
$$

The first order loop has to have a bang-bang angular frequency satisfying both Equations 3.54 and 3.59 to pass jitter tolerance test.

\subsubsection{Jitter Generation}

The jitter generation (TJ) is defined as the total output jitter when there is no input jitter where

$$
T J_{p p}=D J+2 \cdot Q_{B E R} \cdot R J_{r m s}
$$

Where $T J_{p p}$ is the jitter generation, $T J$, expressed in peak-to-peak value.

By definition, jitter generation is measured at $Q_{B E R}=7.0345$ for $B E R=10^{-12}$.

The deterministic jitter is defined as twice the idling pattern peak-to-peak amplitude, and is given by

$$
D J=2 \cdot \omega_{b b} t_{n}=2 \cdot \theta_{b b}
$$


Figure 3.15 shows the sources of noise in the first order loop where $n(s)$ is assumed to a random Gaussian noise associated with the phase of the clock driving the data stream and $\operatorname{Pv}(\mathrm{s})$ is the phase noise of the VCO. The transfer function of the noise loop can be expressed as

$$
\phi_{o}=\frac{H(s)}{s+H(s)} \cdot[n+e]+\frac{s}{s+H(s)} \cdot P_{v}(s)
$$

The transfer function from both random noise source and quantization noise to the output is a low pass filter. However, the transfer function from the VCO phase noise to the output phase is a high pass filter. Therefore, the VCO phase noise is shaped within the loop and filtered out, having minimum effect on the output [1]. In practice, the CDR receiver is designed with a high bandwidth to meet jitter tolerance, thus most of the VCO phase noise is corrected. For example, the SONET required bandwidth is 4 up to $8 \mathrm{MHz}$.

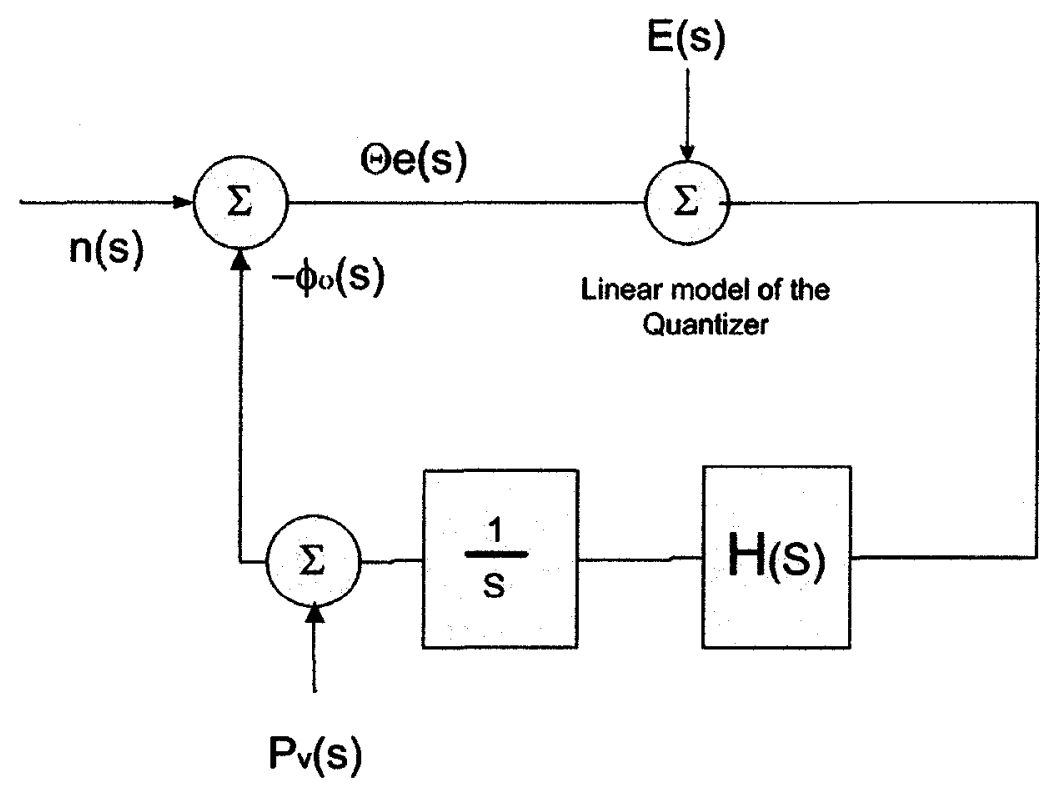

Figure 3.15 First order loop noise model for jitter generation 
The condition of having slope overload with probability $10^{-12}$ is given by Equation 3.15 and substituting $C=7.13$ therefore

$$
\left.\omega_{b b}\right|_{R J} \geq\left.\frac{14.3 \pi t_{n}}{\sqrt{3}}\left(\sigma f_{j b}\right)\right|_{\max }
$$

In order to meet jitter generation (TJ), the first order loop has to have a bang-bang angular frequency satisfying both Equations 3.61 and 3.63.

\subsection{Summary}

The characteristic and parameters of first order loop was modeled using an analogy to the linear Delta Modulation and Sigma Delta Modulation system. The characteristics of idling pattern, dynamic range, and slope overload are defined. Novel equations and conditions were developed to evaluate the signal-to-noise ratio and slope overload condition to Gaussian input jitter at a given bit-error-rate. The analysis was extended to sinusoidal input jitter to calculate signal-to-noise ratio, slope overload conditions and sinusoidal operating conditions. The analysis derived equations and definitions to loop jitter performance in terms of jitter transfer, jitter peaking, jitter tolerance and jitter generation. A complete analysis of phase step response and frequency step response followed by developing expressions for lock-in-phase and lock-in-frequency time. Time domain simulation and behavioral model simulation were used to verify the validity of the analysis in the case of a 5 Gbps CDR. 
The first order loop encounters a narrow lock range $\left\{-f_{b b},+f_{b b}\right\}$. Increasing $f_{b b}$ will increase the lock range but will degrade the jitter performance. This situation can be improved by using the second order loop which is examined in the next chapter. 


\section{Second Order Bang- Bang Clock and Data Recovery}

\subsection{Introduction}

This chapter analyzes the second order bang-bang CDR. The second order loop introduces potential problems of stability which are discussed in detail. It will be shown that the second order loop has a conditional superior signal-to-noise ratio when compared to a first order loop. Four different proposed models based on Double Delta Modulation and second order Sigma Delta Modulation systems will be examined.

Methods of analyzing and describing the second order loop performance detail, as well as perform comparisons between the performance of the first order loop and the second order loop are discussed. The performance of the second order loop is more difficult to analyze when the input is a Gaussian jitter frequency. In sections 4.5 and 4.9 , techniques are used to evaluate the performance for Gaussian signal at a given bit-error-rate. The performance of the loop to sinusoidal input jitter will also be examined in the same section. Jitter performance, as a function of the design loop parameters will be discussed in last three sections. The equations and expressions presented in this chapter were either derived analytically and/or verified with time domain simulation. The graph results are 
presented in this chapter are obtained either from simulation of a second order loop Matlab model or from numerical simulation for some equations. The graph results obtained from simulating the Matlab model are always initialized by word "simulated".

\subsection{Second-Order Loop Dynamics}

The second order loop has a superior jitter performance and wider lock range when compared to the first order loop. It also has an oscillatory behavior which can be contained by applying a simple phase lead correction within the feedback loop.

The second order loop can be implemented by introducing a second integrator into the feedback loop of the first order loop, as shown in Figure 4.1. The $q(t)$ generates positive and negative binary pulses with amplitude $V$. These binary waveforms are integrated by the first integrator to produce a waveform $\theta_{1}(t)$. Each change in $q(t)$ produces a ramp in $\theta_{1}(t)$ with a slope of $V$. A Matlab model follows the diagram shown in Figure 4.1 is utilized to simulate the loop response and validate the equations.

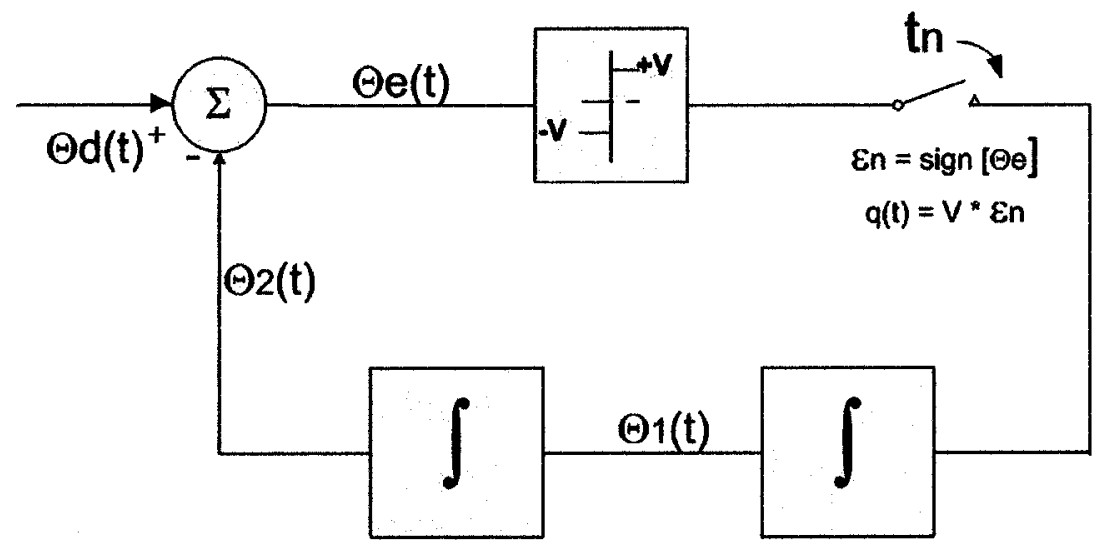

Figure 4.1 Second order Loop Without Phase Lead Correction 
As shown in Figure 4.2, the ramp of $\theta_{1}(t)$ produces a quadratic curve in $\theta_{2}(t)$ which has a value of $1 / 2 f_{n}^{2}$ at the end of a clock period of $t_{n}$.
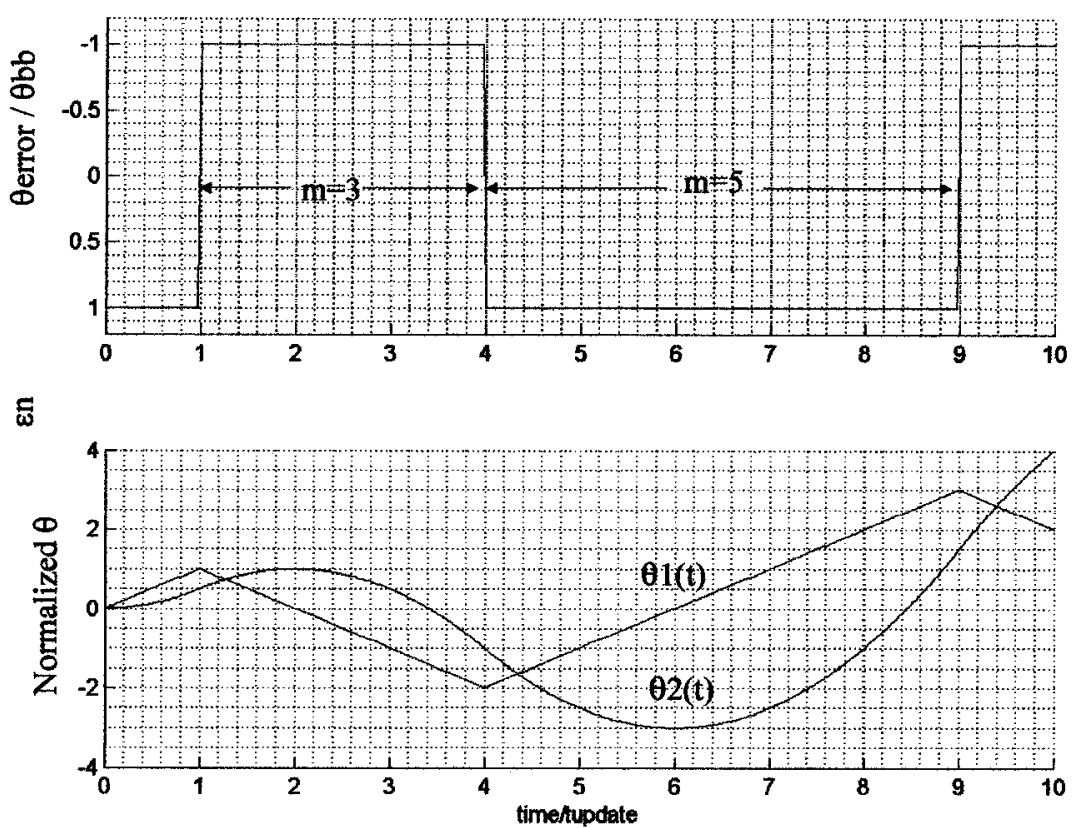

\section{Figure 4.2 Simulated second order loop waveforms when $q(t)$ is series of positive \& negative}

The loop attempts to generate a $\mathrm{q}(\mathrm{t})$ pattern, with a mean value (over a short period of time) that is approximately equal to the mean value of the second derivative of the input phase at the sampling instants.

Figure 4.3 shows how the simulated output phase of the second order loop, without phase lead correction, varies with time when the input phase is sinusoidal. All the waveforms shown are normalized. 

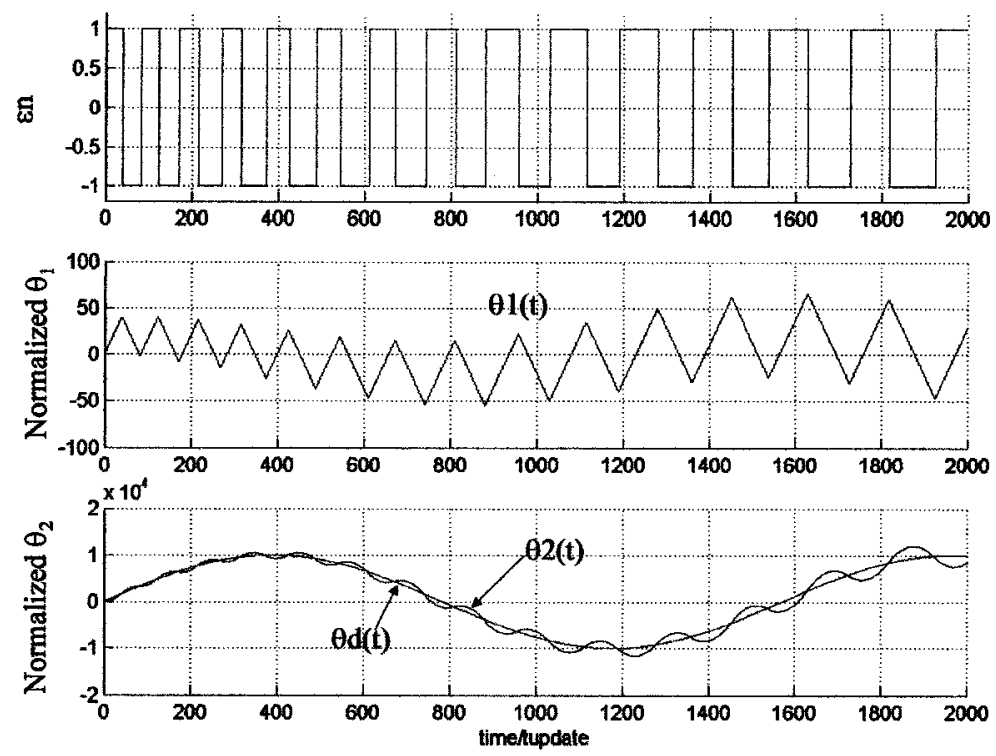

Figure 4.3 Simulated second order loop waveforms when is correctly tracking the input

As expected, the feedback phase $\theta_{2}(t)$ is a much closer representation of the input phase than what is obtained from the first order loop. However, the second order loop is not stable and tends to have oscillatory characteristics when it is in the idling state. These oscillatory characteristics are due to $\theta_{2}(t)$ having maximum slope when crossing zero [44]. Figure 4.4 shows the oscillatory characteristics of normalized $\theta_{2}(t)$ when the second order loop is in the idling state. The oscillation is unstable and grows in amplitude, with peaks occurring at $\left.t_{2}\right|_{p e a k}$ From simulation, we found $\left.t_{2}\right|_{p e a k}$ to follow the formula

$$
\left.t_{2}\right|_{\text {peak }}=\frac{t_{n}}{4} \cdot\left(m_{i}^{2}-1\right)
$$


with peak values of $\left.\theta_{2}\right|_{p e a k}$, given by

$$
\left.\theta_{2}\right|_{p e a k}=\frac{V f_{n}^{2}}{8} \cdot\left(m_{i}^{2}-1\right)
$$

where $m_{i}$ is the number of cycles of sampling instant $\left(t_{n}\right)$ before the loop alternates the phase error direction as illustrated in Figure 4.4. The value of $m$ determines the order of the idling mode, i.e., $m_{i}=2$ means the second order idling mode and $m_{i}=3$ is the third order idling mode. The idling order mode is defined as the number of cycles of $t n$ between two consecutive zero crossing of output phase, $\theta_{2}(t)$. When in the idling state, the loop starts with a lower order mode $\left(m_{i}=1\right.$ or $\left.m_{i}=2\right)$ which then gradually increases to higher order modes. Therefore, the idling oscillation does not have a fixed frequency, unlike the first order loop whose idling oscillation has a frequency of $f_{n} / 2$. With analogy to Delta Modulation, the loop does not necessarily start with the lowest order mode, since $\mathrm{m}$ is a function of the initial condition [44]. However, the loop still starts in a lower mode and then moves toward higher order modes. 


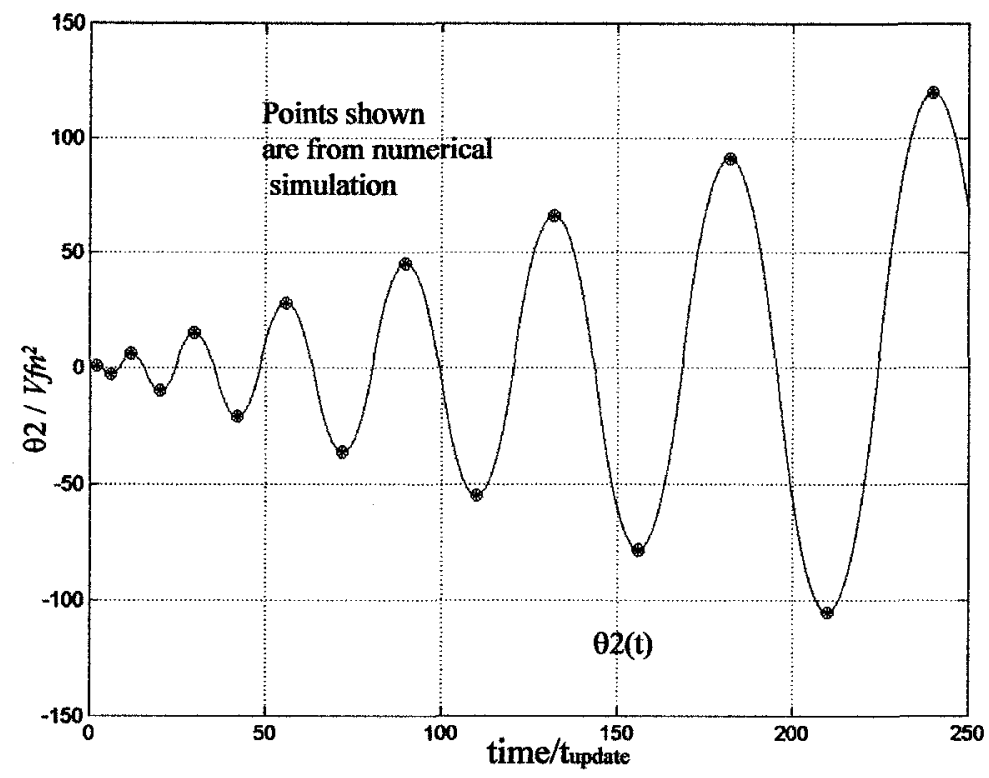

Figure 4.4 Simulated second order loop in idling state

The second order loop might be stable if the input phase slowly changes its slope with time. However, any rapid changes may cause the loop to have oscillatory properties. The oscillation of the second order loop must be damped by introducing a phase lead correction means, placed within the feedback loop.

\subsection{Stabilizing the Second Order Loop}

In order to eliminate the oscillatory proprieties for the second order loop, a simple phase lead correction is used with the first integrator as shown in Figure 3.9. The output wave form is $\theta_{3}(t)$, where

$$
\theta_{3}(t)=\int \theta_{1} d t+\frac{\beta}{\tau} \cdot \int q(t) d t
$$




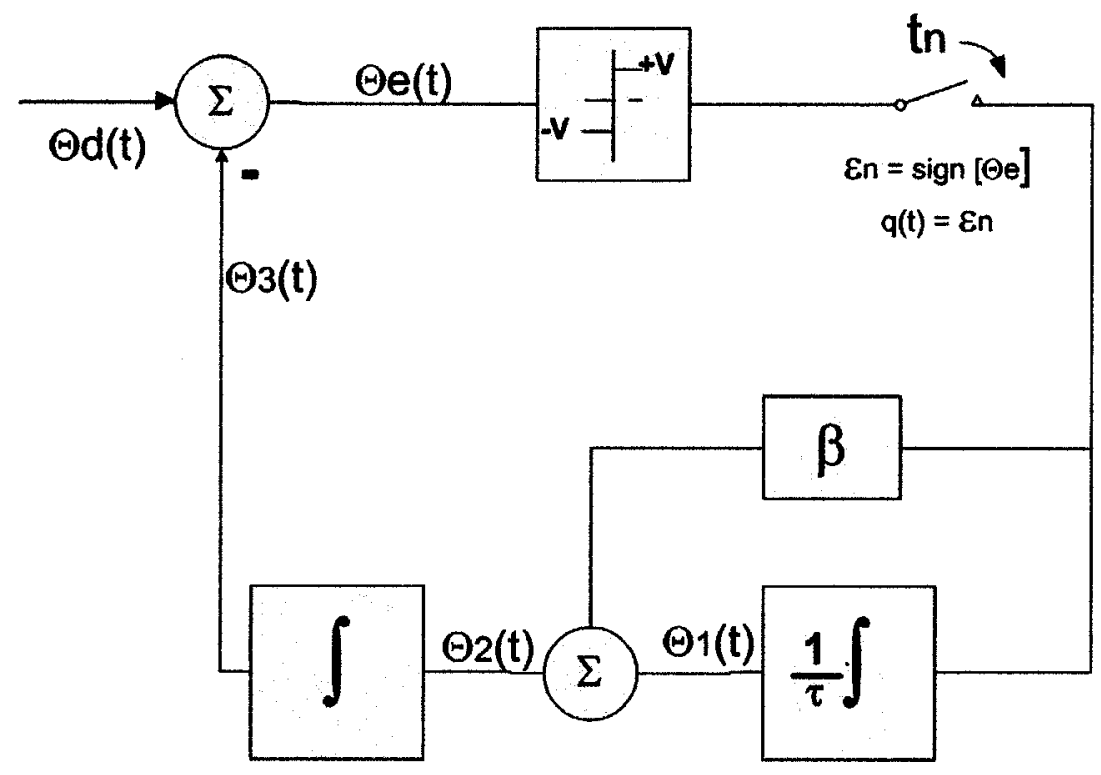

Figure 4.5 Second order loop with phase lead correction

$q(t)$ generates pulses that represent the phase error direction, with each pulse immediately changing $\theta_{2}(t)$ by $\varepsilon_{n} \cdot \frac{\beta}{\tau}$ during one update time. The step change in $\theta_{2}(t)$ produces a ramp in phase of $\theta_{3}(t)$ in the direction of the phase error, which directly minimizes the phase error. For convenience, this report adopts the same definition of loop stability factor $\zeta$ first introduced by Walker (Equation 2.16). With analogy to Double Delta Modulation, the value of the stability factor which is necessary to damp the idling oscillation is given by [44]

$$
\xi \geq \frac{m_{i}}{m_{i}-2} \quad m_{i}>2
$$

Figure 4.6 shows the undamped waveform against a damped waveform with a stability factor value of $\xi=1$. The oscillation with $\mathrm{m}_{\mathrm{i}}=11$ has been damped to oscillation 
with $m_{i}=4$. The use of the phase lead correction pushes the higher order mode $m_{i}$ toward the lowest order value of $m_{i}=1$ or $m_{i}=2$. To ensure that the higher order oscillatory idling waveforms are damped to $m_{\mathrm{i}}=1$ or $\mathrm{m}_{\mathrm{i}}=2$, a stability factor has to be chosen to have a greater value than required by Equation 4.4 .

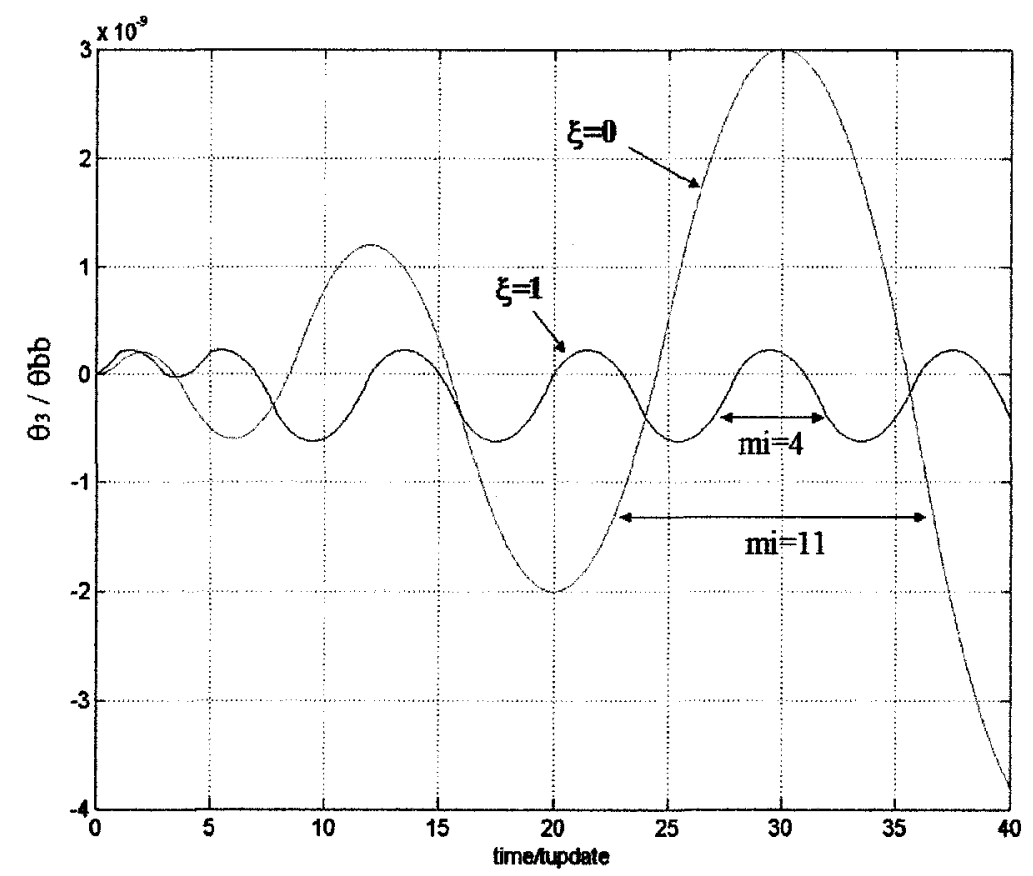

Figure 4.6 Simulated the effect of applying phase lead correction with minimum stability factor to second order loop

The lower the order mode, the higher the stability factor required to damp it to $m=1$ or $m_{i}=2$. Therefore, the minimum value of the stability factor required to make the loop just stable is $\left.\xi\right|_{\min }=1$. Figure 4.7 shows waveforms for a second order loop with different values of stability factors that are less than minimum required $\left.\xi\right|_{\min }=1$. None of them was able to damp the idling oscillation. 


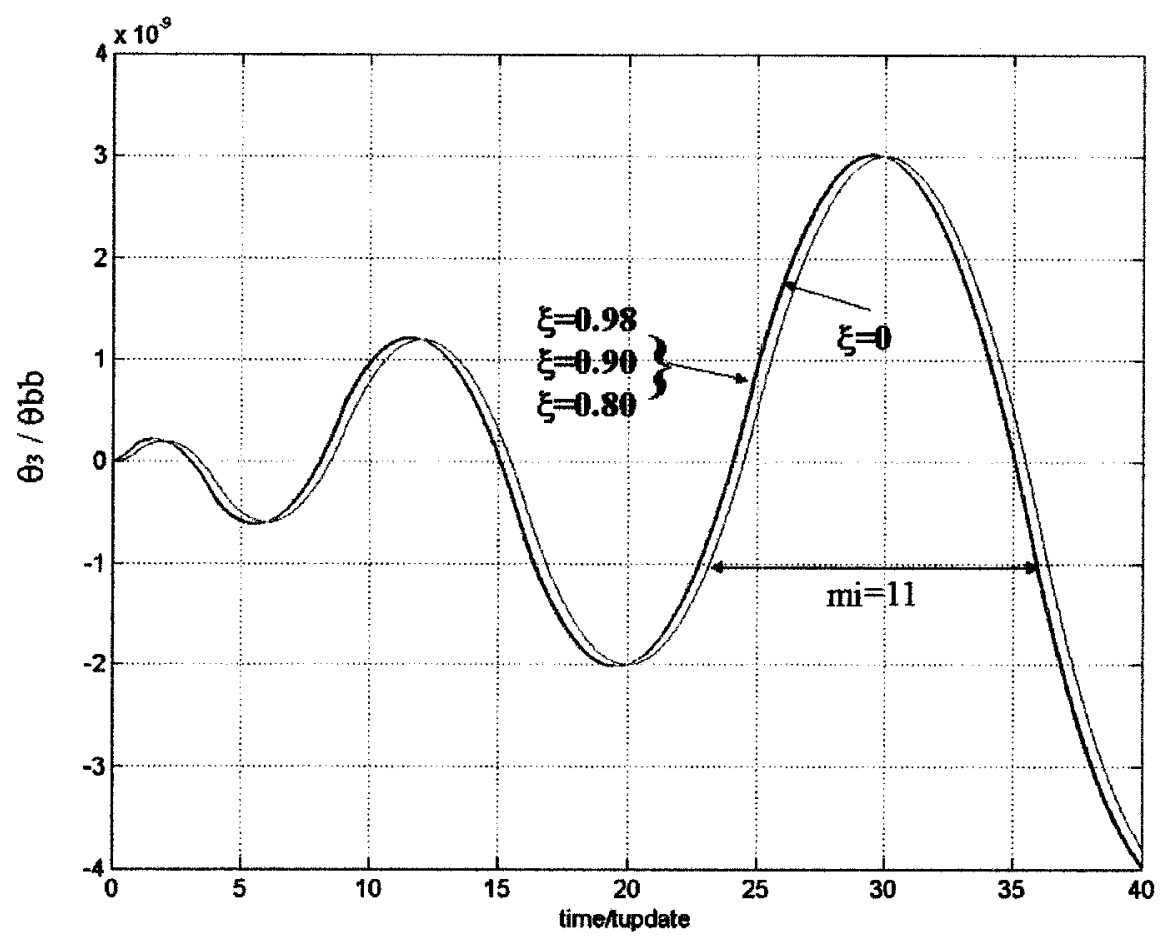

Figure 4.7 Simulated the effect of applying stability factor $<1$ to second order loop

A stability factor value of $\xi=3$ is enough to damp all lower and higher modes to the lowest modes $\left(m_{i}=1\right.$ or $\left.m_{i}=2\right)$. For greater values of $\xi$ than required by the Equation 4.4, the loop eliminates the transient phase error and settles faster. However, with increasing values of $\xi$, the signal-to-quantization noise ratio degrades and the stationary phase error increases, as shown in Figure 4.8. 


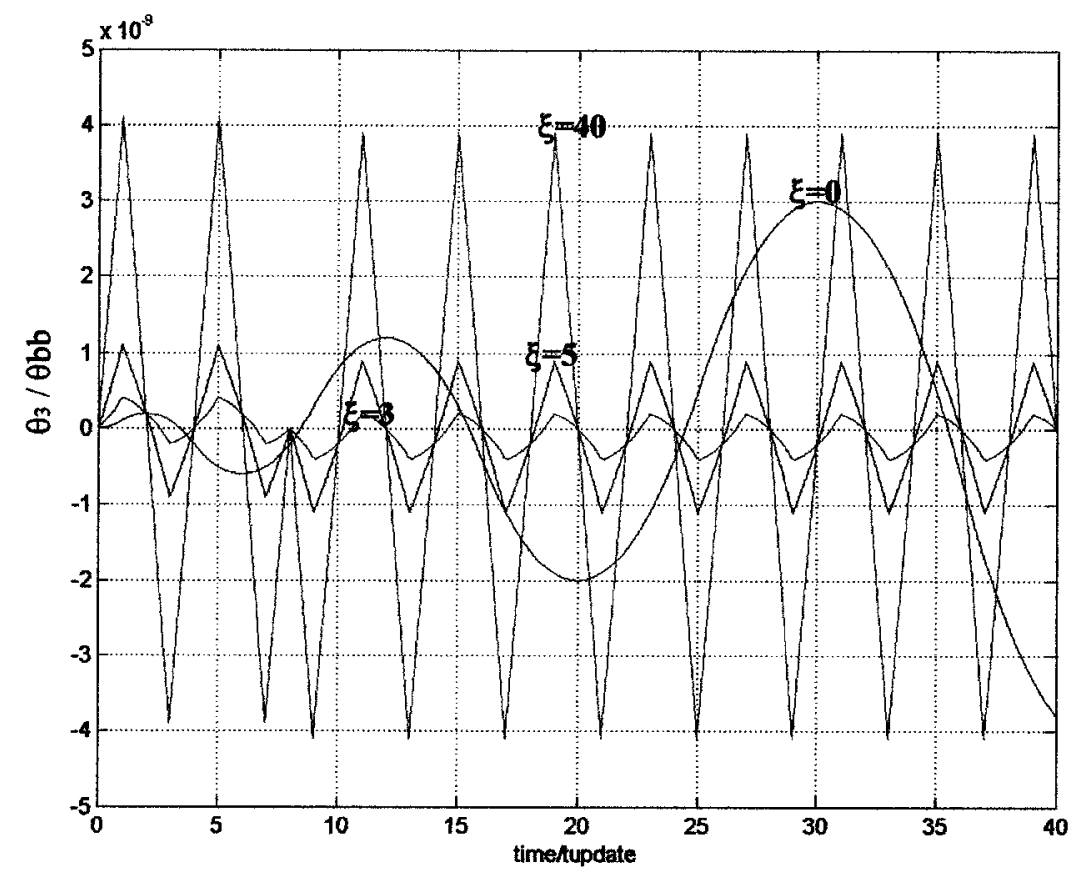

Figure 4.8 Simulated the effect of increasing stability factor value on second order loop in its idling state

When the stability factor is selected to be less than that required by Equation 4.4 but greater than the minimum $\left.\xi\right|_{\min }=1$, the higher order mode waveforms will be damped to lower order modes but not to $m_{i}=1$ or $m_{i}=2$ as shown in Figure 4.6. The required $\xi$ to damp $m_{i}=11$ to $m_{i}=1$ is $\left.\xi\right|_{11} \geq 1.23$, however, when the loop uses $\xi=1$ the oscillations were damped to $\mathrm{m}_{\mathrm{i}}=4$.

In light of the above discussion, we can explain why the second order loop experiences wander when the input data has a long-run pattern, at which case the loop acts as a second order loop with no input phase. As we explained earlier, depending on the stability factor, such a loop may have oscillatory properties which may grow in amplitude over the 
limits of dynamic range of the phase detector. In order to eliminate this phenomenon, a higher stability factor is needed.

\subsection{Model of Second-Order Loop}

The second order loop can be modeled by inserting the integration path within the first order loop as shown in Figure 4.9, where $K_{c p}$ is the integration path gain.

In the same way, the model assumes the loop has uniform sampling times $\left(t_{n}\right)$ and a ternary phase detector. The input phase $\theta d$ is the sum of the phase component due to frequency offset, and the phase shift component $\theta_{d}\left(t_{m}\right)=\delta \omega \cdot t_{m}+\phi\left(t_{m}\right)$ at $\mathrm{m}^{\text {th }}$ sampling time $t_{n}$

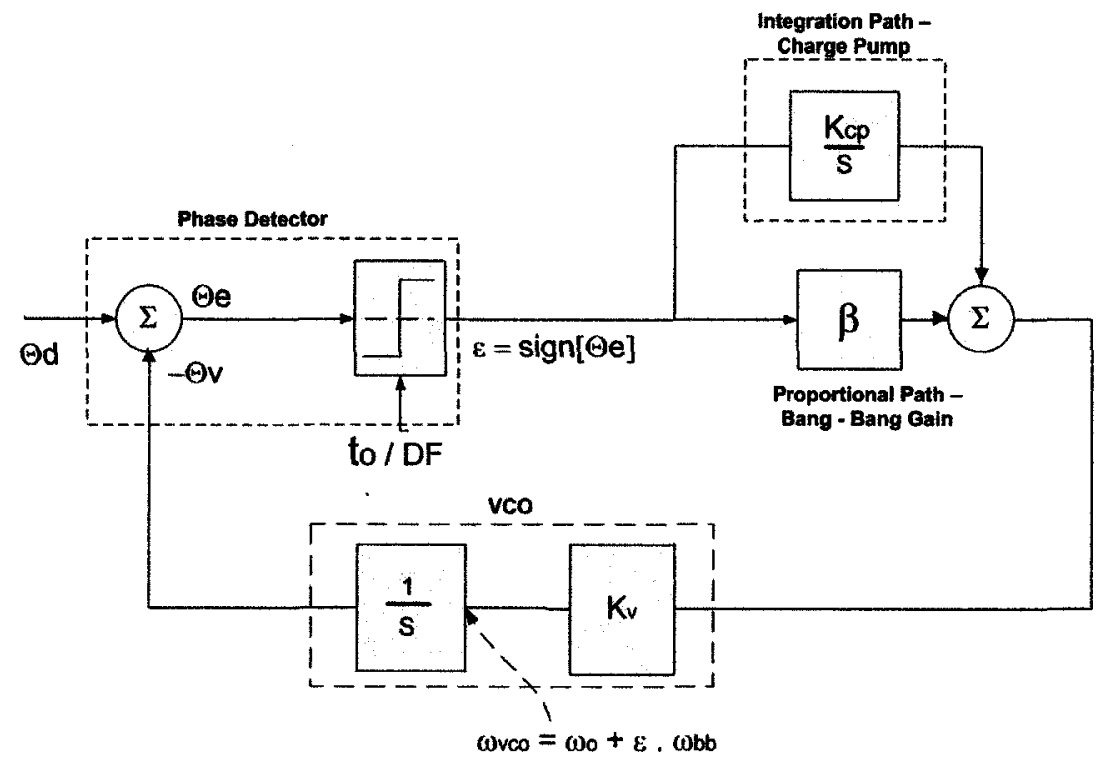

Figure 4.9 Second order bang-bang loop model based on linear Delta Modulation

The set of discrete time difference equations describing the second order loop dynamics evaluated at each $\mathrm{m}^{\text {th }}$ sampling time $t \boldsymbol{n}$ is given by 


$$
\theta_{d}\left(t_{m}\right)=\theta_{d}(0)+\delta \omega t_{m}+\phi_{d}\left(t_{m}\right)
$$

$$
\varepsilon_{m}=\operatorname{sign}\left(\theta_{d}\left(t_{m}\right)-\theta_{v}\left(t_{m}\right)\right)
$$

where time $t_{m}$ at $\mathrm{m}^{\text {th }}$ sampling time is defined as

$$
\begin{gathered}
t_{m}=m \cdot t_{n} \\
\theta_{v}\left(t_{m+1}\right)=\theta_{v}\left(t_{m}\right)+\int_{m \cdot t n}^{(m+1) t n} \varepsilon_{m} \beta K_{v} d t+\int_{m \cdot t n}^{(m+1) t n} \varepsilon_{m} K_{c p} K_{v} d t
\end{gathered}
$$

Therefore,

$$
\theta_{v}\left(t_{m+1}\right)=\theta_{v}\left(t_{m}\right)+\varepsilon_{m} \beta K_{v} t_{n}+\varepsilon_{m} K_{c p} K_{v} \cdot \int_{(\dot{m} \cdot t n)}^{(m+1) t n} t d t
$$

Substituting the values of $\theta_{b b}$ and $\xi$ from Equation 3.28 and 2.16 respectively, we get

$$
\theta_{v}\left(t_{m+1}\right)=\theta_{v}\left(t_{m}\right)+\varepsilon_{m} \theta_{b b}+\varepsilon_{m} \cdot \frac{2 \theta_{b b}}{\xi \cdot t_{n}^{2}} \cdot \int_{(m \cdot t n)}^{(m+1) t n} t d t
$$

and consequently,

$$
\theta_{v}\left(t_{m+1}\right)=\theta_{v}\left(t_{m}\right)+\varepsilon_{m} \theta_{b b}+\varepsilon_{m} \cdot \frac{\theta_{b b}}{\xi \cdot t_{n}^{2}} \cdot\left[(m+1)^{2}-m^{2}\right] t^{2}{ }_{n}
$$


which reduces to

$$
\theta_{v}\left(t_{m+1}\right)=\theta_{v}\left(t_{m}\right)+\varepsilon_{m} \theta_{b b}+\varepsilon_{m} \cdot \frac{\theta_{b b}}{\xi} \cdot[2 m+1]
$$

which when simplified becomes

$$
\theta_{v}\left(t_{m+1}\right)=\theta_{v}\left(t_{m}\right)+\varepsilon_{m} \theta_{b b} \cdot\left[1+\frac{1}{\xi}+\frac{2 m}{\xi}\right]
$$

Figure 4.10 shows the Delta Sigma Modulation model of the second order loop when the input phase is due to a frequency offset component $\delta \omega \cdot t_{n}$ and with $\phi\left(t_{m}\right)=0$. The model's discrete time difference equations are

$$
\begin{aligned}
& \varepsilon_{m}=\operatorname{sign}\left(\int_{m t n}^{(m+1) t n}\left(\delta \omega-\omega_{v}\left(t_{m}\right)\right) d t\right) \\
& \omega_{v}\left(t_{m+1}\right)=\omega_{v}\left(t_{m}\right)+\varepsilon_{m} \omega_{b b} \cdot\left[1+\frac{2}{\xi}\right]
\end{aligned}
$$




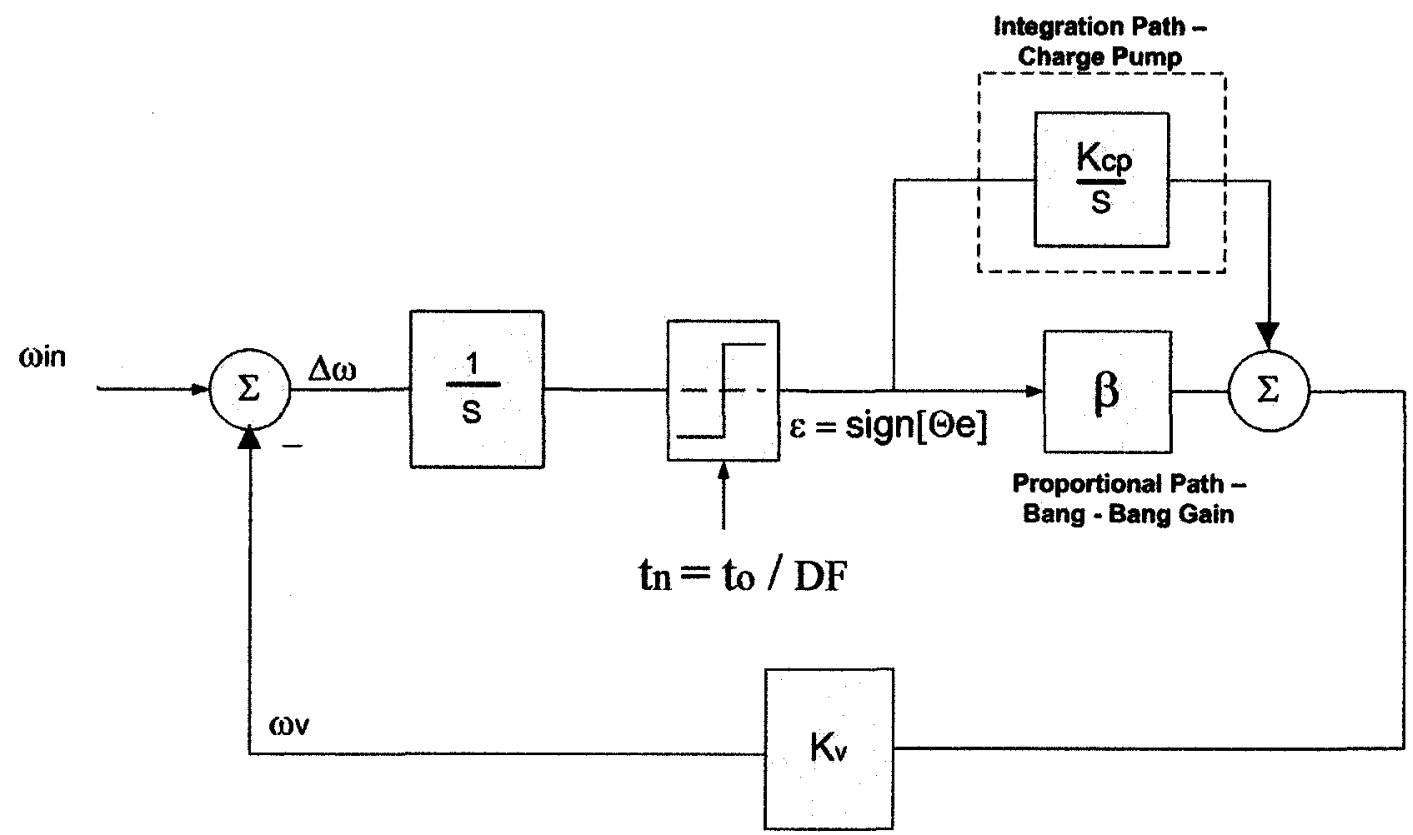

Figure 4.10 Second order Bang-Bang loop model based on Delta Sigma Modulation

\subsubsection{Second Order Bang-bang Linearized Model}

The model of the second order loop based on Double Delta Modulation can be linearized as shown in Figure 4.11, where the quantizer is replaced with the linear model and an error source. The model is valid if the loop does not experience a slope overload. The transfer function of the loop is given by

$$
\theta_{v}(s)=P T R \cdot \theta_{d}(s)+N T R \cdot E(s)
$$


where

$$
P T R=N T R=\frac{\omega_{b b} S_{h} \cdot s+\frac{2}{\xi} \cdot f_{n} \omega_{b b} S_{h}}{s^{2}+\omega_{b b} S_{h} \cdot s+\frac{2}{\xi} \cdot f_{n} \omega_{b b} S_{h}}
$$

for $\lim _{\xi \rightarrow \infty} P T R=\frac{\omega_{b b} S_{h}}{s+\omega_{b b} s_{h}} \quad$ equals to the first order loop Equation 3.33.

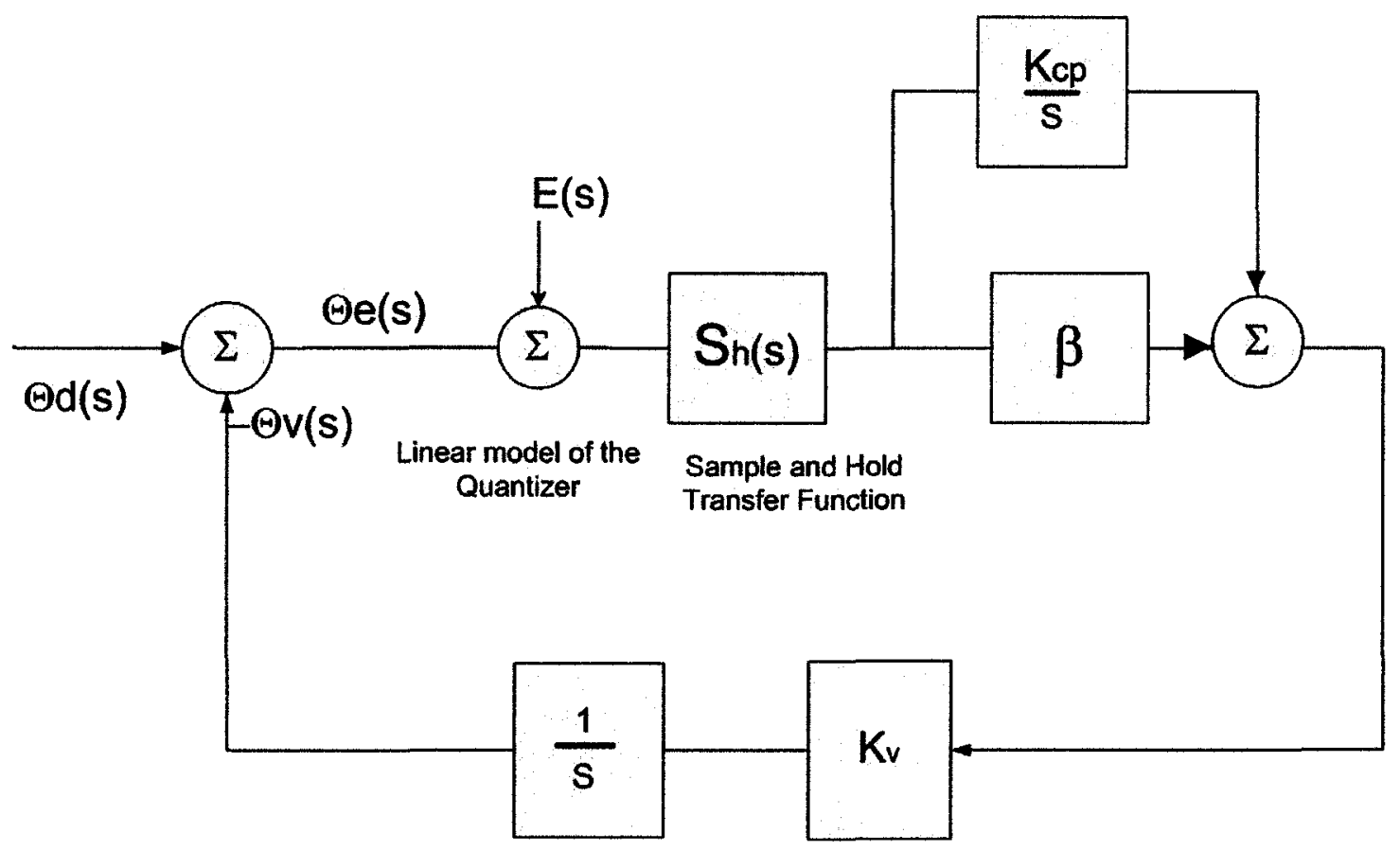

Figure 4.11 Mathematical model for analyzing second order loop employing a linear model for quantizer

Similarly, the Delta Sigma Modulation linearized model of the second order bangbang loop is shown in Figure 4.12. 


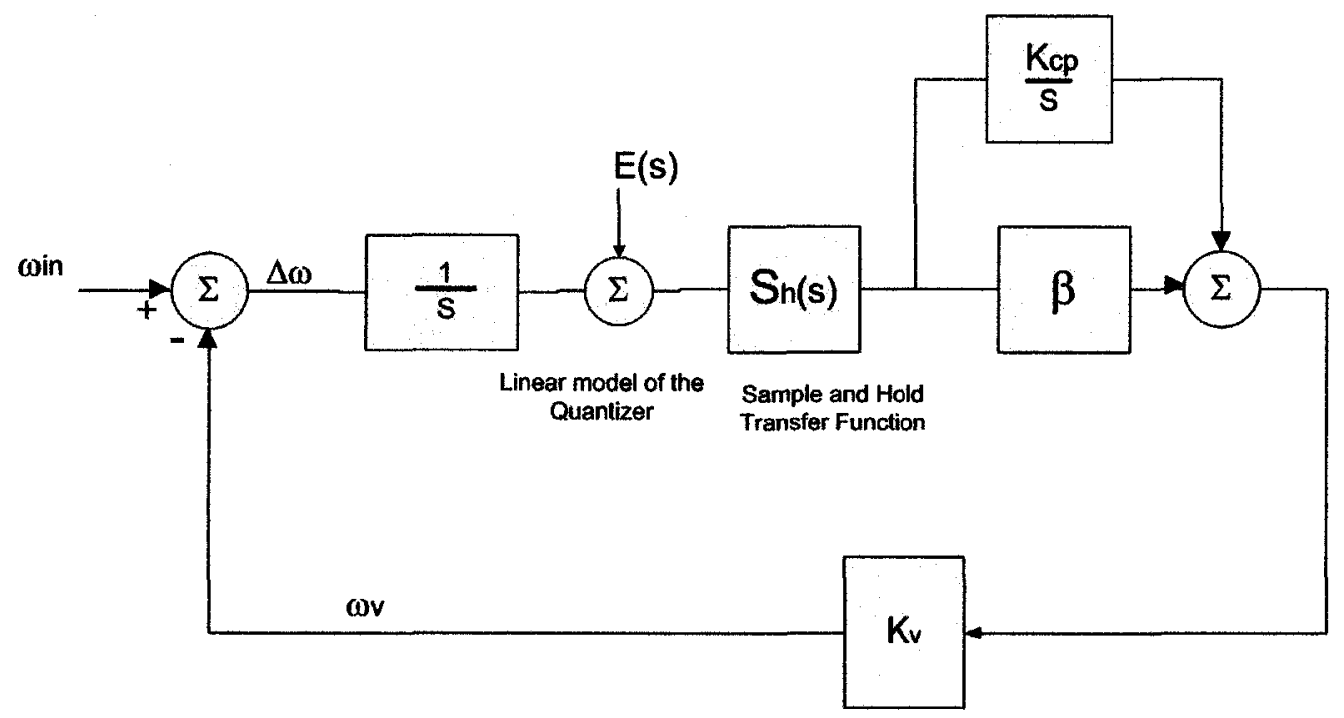

Figure 4.12 Mathematical model for analyzing second order loop employing a linear model for quantizer when the frequency offset is considered

The transfer function of the above loop is

$$
\omega_{v}(s)=F T R \cdot \omega_{\text {in }}+N T R \cdot E(s)
$$

where FTR is the frequency transfer function

$$
\begin{gathered}
F T R=\frac{N T R}{s}=\frac{\omega_{b b} S_{h} \cdot s+\frac{2}{\xi} \cdot f_{n} \omega_{b b} S_{h}}{s^{2}+\omega_{b b} S_{h} \cdot s+\frac{2}{\xi} \cdot f_{n} \omega_{b b} S_{h}} \\
N T R=\frac{\omega_{b b} S_{h} \cdot s^{2}+\frac{2}{\xi} \cdot f_{n} \omega_{b b} S_{h} \cdot s}{s^{2}+\omega_{b b} S_{h} \cdot s+\frac{2}{\xi} \cdot f_{n} \omega_{b b} S_{h}}
\end{gathered}
$$


The quantization noise has negligible effect on output frequency spectrum due to the shaping of the quantization noise by the loop and since the quantization noise inside the loop bandwidth is filtered out by the high pass filter.

\subsection{Second Order Loop Slope Overload}

When the loop is correctly tracking the input phase, the phase error varies around the input phase $\theta_{d}(t)$ and is always bounded by $\left|\theta_{e}(t)\right| \leq \theta_{b b}\left[1+\frac{1}{\xi}+\frac{2 m}{\xi}\right]$, where $m$ is the cycle number of $t n$. However, when the slope of the input phase increases, the loop attempts to generate a sequence of equal polarity pulses to minimize the phase error. A Matlab model follows the diagram shown in Figure 4.9 is utilized to simulate the response and validate the equations. As shown in Figure 4.13, if the slope of the input phase increases to a value greater than a maximum slope of $\theta_{v}(t)$, then the loop will fail to track the input phase and start to experience a slope overload. Hence, the phase error will increase beyond the limit given above.
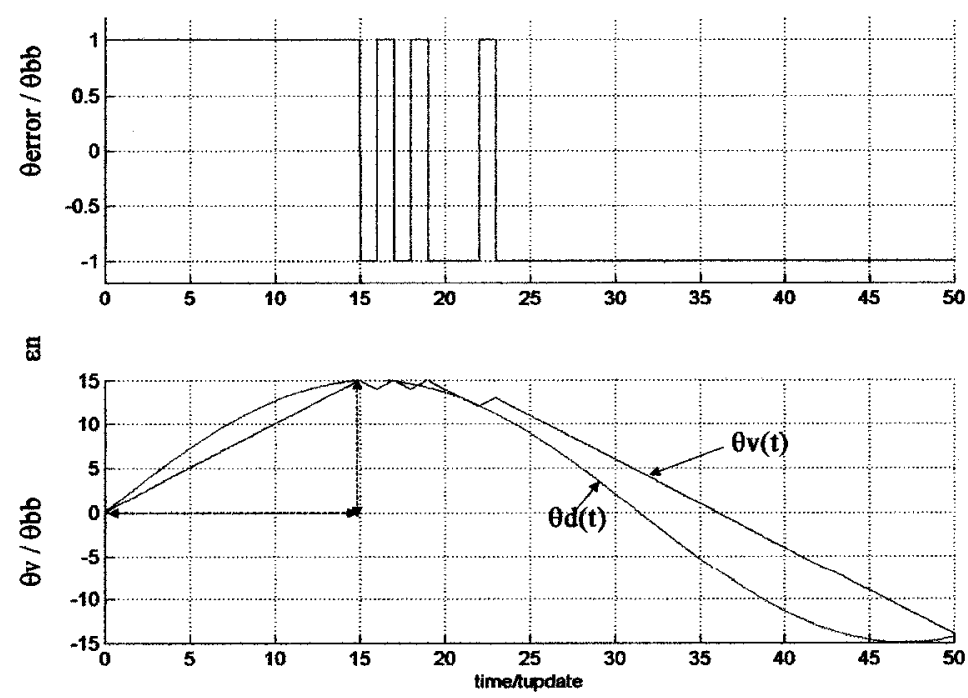

Figure 4.13 Simulated normalized output phase waveform when the second order loop has experienced slope overload 


\subsubsection{The Maximum Slope of the Output Phase}

When the output phase generates a sequence of equal polarity pulses, the loop phase change due to one update time can be determined from Equation 4.13 as

$$
\Delta \theta(m)=\varepsilon_{m} \theta_{b b} \cdot\left[1+\frac{1}{\xi}+\frac{2 m}{\xi}\right]
$$

For $\mathrm{m}=\mathbf{0}$,

$$
\begin{aligned}
& \Delta \theta(0)=\theta_{b b} \cdot\left[1+\frac{1}{\xi}\right] \\
& \Delta \theta(1)=\theta_{b b} \cdot\left[1+\frac{3}{\xi}\right] \\
& \Delta \theta(2)=\theta_{b b} \cdot\left[1+\frac{5}{\xi}\right]
\end{aligned}
$$

Substituting Equation 4.22 into 4.13 to obtain the output phase, we get:

$$
\theta_{v}\left(t_{0}\right)=\theta_{b b} \cdot\left[1+\frac{1}{\xi}\right]
$$

We can obtain the output phase at $t_{1}$ by adding 4.25 and 4.23

$$
\theta_{v}\left(t_{1}\right)=\theta_{b b} \cdot\left[2+\frac{4}{\xi}\right]
$$

And for $t_{2}$, the output phase is obtained by adding 4.26 and 4.24 


$$
\theta_{v}\left(t_{2}\right)=\theta_{b b} \cdot\left[3+\frac{9}{\xi}\right]
$$

The output phase at $t_{m+1}$ is therefore

$$
\theta_{v}\left(t_{m+1}\right)=\theta_{b b} \cdot\left[m+\frac{m^{2}}{\xi}\right]
$$

By substituting $m=\frac{t}{t_{n}}$ and differentiating, the maximum slope of the output phase is obtained as

$$
\theta_{v}^{\prime}\left(t_{m+1}\right)=\omega_{b b} \cdot\left[1+2 \frac{m}{\xi}\right]
$$

\subsubsection{Slope Overload due to DJ}

Assuming the input jitter is sinusoidal and is evaluated at each update time $\left(t_{n}\right)$, then the maximum rate of increase of the input is

$$
\left.\theta_{d}^{\prime}\left(t_{n}\right)\right|_{\max }=m_{j} \cdot \omega_{j}
$$

The condition for slope overload can be obtained from Equations 4.29 and 4.30

$$
m_{j} \cdot \omega_{j} \leq \omega_{b b} \cdot\left[1+2 \frac{m}{\xi}\right]
$$


The second order loop can track a higher frequency jitter than the first order loop can, without incurring a slope overload condition. Unlike the first order loop, the bangbang angular frequency step can be used in addition to the stability factor to allow the loop to correctly track the incoming input jitter.

Figure 4.13 shows the second order loop operating in the slope overload condition with a sinusoidal input jitter. Whenever the input frequency jitter decreases, the required number of cycles $m$ increases and vice-versa. The number of cycles $m$ is a function of the input jitter frequency and it is always less than $\frac{f_{n}}{4 f_{j}}$.

When putting

$$
m=r \cdot \frac{\omega_{n}}{4 \omega_{j}}
$$

where $r$ is an arbitrary number less than unity, we obtain

$$
m_{j} \cdot \omega_{j} \leq \omega_{b b} \cdot\left[1+r\left(\frac{\omega_{n}}{2 \omega_{j} \xi}\right)\right]
$$

Then,

$$
m_{j} \cdot \omega_{j}^{2}-\omega_{b b} \omega_{j} \leq \frac{r \omega_{b b} \omega_{n}}{2 \xi}
$$


For $\omega_{b b}=0$, the second order loop has no phase lead correction and generates pulses with rates of occurrence proportional to the second derivative of the input jitter. At this condition, the slope overload condition can be expressed, by definition, as

$$
\omega_{j}^{2} \cdot m_{j} \leq K_{v} K_{c p} t_{n}
$$

To find the value of $r$, we substitute Equation 4.35 into 4.34 when $\omega_{b b}=0$

$$
r=\frac{2}{\pi}
$$

Substituting Equation 4.36 into 4.34 to find the slope overload condition for the second order loop, we get

$$
m_{j} \cdot \omega_{j}^{2}-\omega_{b b} \omega_{j} \leq \frac{\omega_{b b} \omega_{n}}{\pi \xi}
$$

\subsubsection{Slope Overload due to RJ}

Similar to the first order loop, the probability of having slope overload due to random jitter in the second order loop, where the jitter is defined as a band-limited Gaussian distribution from 0 to $f j b \mathrm{~Hz}$ and $\sigma^{2}$, is the mean square value. The general condition of the slope overload is generally given by substituting Equation 4.29 into 3.14.

$$
C \cdot \sigma_{d} \leq \omega_{b b} \cdot\left[1+2 \frac{m}{\xi}\right]
$$

From Equations 3.15 and 4.38 we get 


$$
C \cdot \sigma \leq \sqrt{3} \frac{\omega_{b b}}{\omega_{j b}} \cdot\left[1+2 \frac{m}{\xi}\right]
$$

When the loop has no proportional path $\left(\omega_{b b}=0\right)$, the second order loop generates pulses with rates of occurrence proportional to the second derivative of the input jitter.

The slope overload condition of second order loop with no phase lead correction is then

$$
C \cdot \sigma_{d d} \leq K_{c p} K_{v} t_{n}
$$

where $\sigma_{d d}$ is the mean square value of the second derivative of input phase $\theta_{d}$. Therefore,

$$
\sigma_{d d}^{2}=\rho_{-f j b}^{j b}(2 \pi)^{4}\left(\frac{\sigma^{2}}{2 f j b}\right) d f
$$

$\left(\frac{\sigma^{2}}{2 f j b}\right)$ is the spectral density function of band limited white noise, and $(2 \pi f)^{4}$ is the power transfer function of second order differentiation. Hence,

$$
\sigma_{d d}=\frac{1}{\sqrt{5}} \cdot \omega_{j b}^{2} \cdot \sigma
$$

Consequently,

$$
C \cdot \sigma \leq \sqrt{5} \frac{K_{c p} K_{v}}{\omega_{j b}{ }^{2}} t_{n}
$$


Substituting $\omega_{b b}=0$ into Equation 4.39, we get

$$
C \cdot \sigma \leq \sqrt{3} \frac{K_{c p} K_{v}}{\omega_{j b}} \cdot t
$$

From Equations 4.43 and 4.44, we can obtain the value of $t$ as

$$
t=\sqrt{\frac{5}{3}} \cdot \frac{t_{n}}{\omega_{j b}}
$$

Since $m=\frac{t}{t_{n}}$,

$$
m=\sqrt{\frac{5}{3}} \cdot \frac{1}{\omega_{j b}}
$$

Substituting Equation 4.46 into Equation 4.39 to find the general slope overload condition of the second order loop, we get

$$
C \cdot \sigma \cdot \omega_{j b}^{2}-\sqrt{3} \omega_{b b} \omega_{j b} \leq 2 \sqrt{5} \frac{\omega_{b b}}{\xi}
$$

Therefore

$$
\begin{gathered}
\sigma_{\text {max }}=\frac{1}{C \cdot \omega^{2}{ }_{j b}} \cdot\left[2 \sqrt{5} \frac{\omega_{b b}}{\xi}+\sqrt{3} \omega_{b b} \omega_{j b}\right] \\
\sigma_{\max }=\frac{1}{C \cdot f^{2}{ }^{\prime} b} \cdot\left[\sqrt{5} \frac{f_{b b}}{\pi \xi}+\sqrt{3} f_{b b} f_{j b}\right]
\end{gathered}
$$




\subsection{Phase Step Response}

Assume the second order loop to be in a lock condition. In this section, a Matlab model follows the diagram shown in Figure 4.9 is utilized to simulate the loop response and validate the equations. We further assume that $\delta \omega=0$ and the input phase is a step function $\phi=\operatorname{\theta r}(t)$ at $t=0$ where $\theta$ is the size of the phase step. The loop would then be experiencing a slope overload, leading to a transient phase error. The phase error increases at the maximum rate at each update time, $t_{n}$, until the slope of the output phase flips its sign. From Equation 4.28, the phase error can be expressed as

$$
\theta_{\text {error }}(t)=\theta-\theta_{b b} \cdot\left[m+\frac{m^{2}}{\xi}\right]
$$

From the above equation, the time of the phase error's first zero crossing is

$$
\frac{t}{t_{n}}=m=\xi \cdot\left(\frac{-1+\sqrt{1+\frac{4}{\xi} \frac{\theta}{\theta_{b b}}}}{2}\right)
$$

As $\xi \rightarrow \infty$, the second order loop becomes a first order loop and the above Equation becomes identical to Equation 3.36 .

$$
\frac{t}{t_{n}}=m=\frac{\theta}{\theta_{b b}}
$$

For any other value of $\xi$, the second order loop has $m<\frac{\theta}{\theta_{b b}}$ where the minimum $m$ is found when the loop has the minimum stability factor $\xi=1$. Then 


$$
m_{\min }=\left(\frac{-1+\sqrt{1+4 \frac{\theta}{\theta_{b b}}}}{2}\right)
$$

The second order loop is quicker to reach zero crossing phase error than the first order. However the second order loop experiences an oscillatory overshoot. The phase transient response of the second order loop due to the input phase step amplitude experiences an overshoot in respect of the linear transient response of the first order loop, while at the same time being bounded by the input phase step when the loop is stable as shown in Figure 4.14. The first order loop step response requires a shorter time to reach the steady state than the second order loop. The second order loop with a high stability factor requires less time than the loop with a low stability factor.

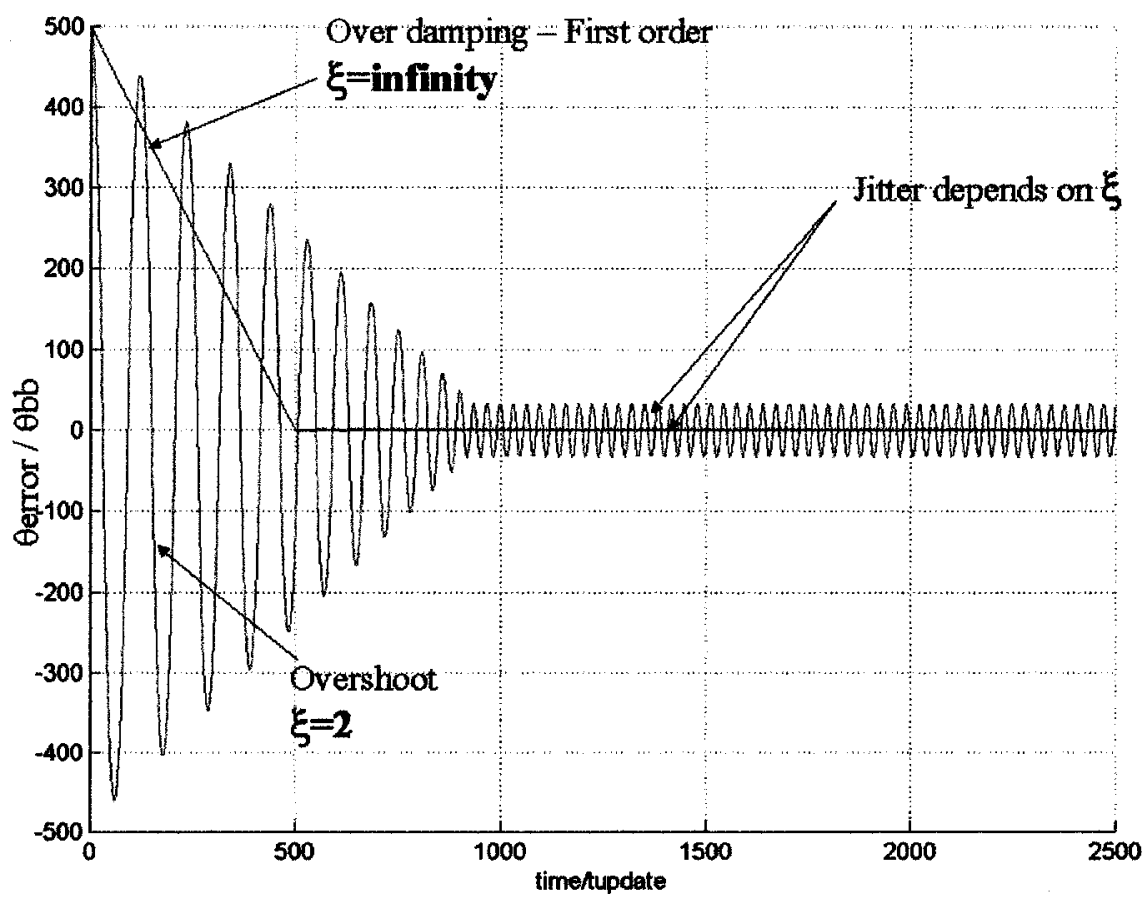

Figure 4.14 Simulated responses of second and first order loops to phase step 
For the second order loop to track the high speed input jitter without having an impact on the bit-error rate, the overshoot properties have to be damped. The $\xi$ required to make the second order loop step response to approximately act like the first order response is

$$
\xi>4 \frac{\theta}{\theta_{b b}}
$$

As shown in Figure 4.15, the simulation of normalized phase error shows an agreement with Equations 4.51 and 4.54. The loop is simulated with different stability factors to obtain the overshoot amplitude and the time required by the loop to eliminate the phase error. The oscillation is partially damped when $\xi=\frac{\theta}{\theta_{b b}}$ and completely damped when $\xi=4 \frac{\theta}{\theta_{b b}}$. For example, the loop with $\xi=200$ and $\frac{\theta}{\theta_{b b}}=50$ has a linear transient response and does not experience overshoot properties. 


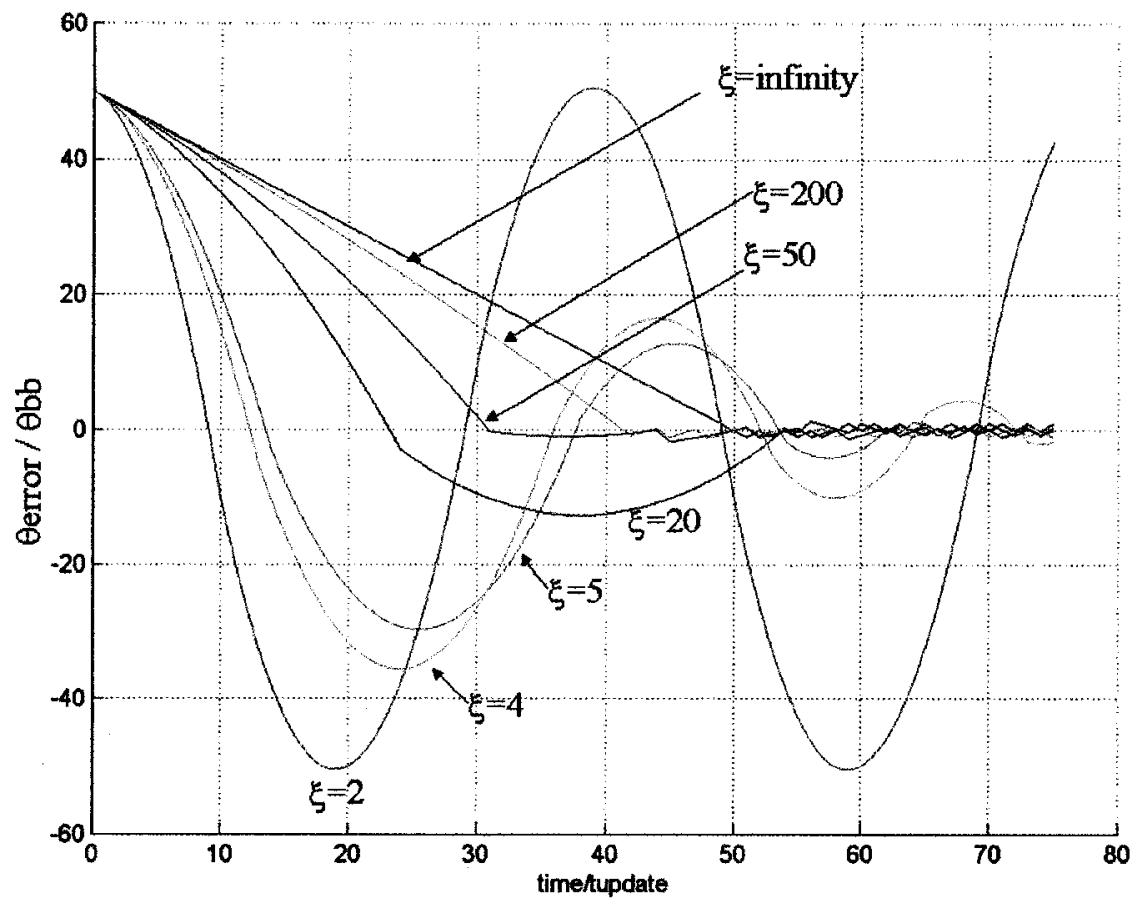

Figure 4.15 Simulated response of second order loop to phase step with range of stability factors

Figure 4.16 shows the simulation of normalized phase error response with a range of input phase step amplitudes. The overshoot amplitude decreases and can be eliminated (if $\xi=\frac{\theta}{\theta_{b b}}$ ) with the decrease of input phase step amplitude. 


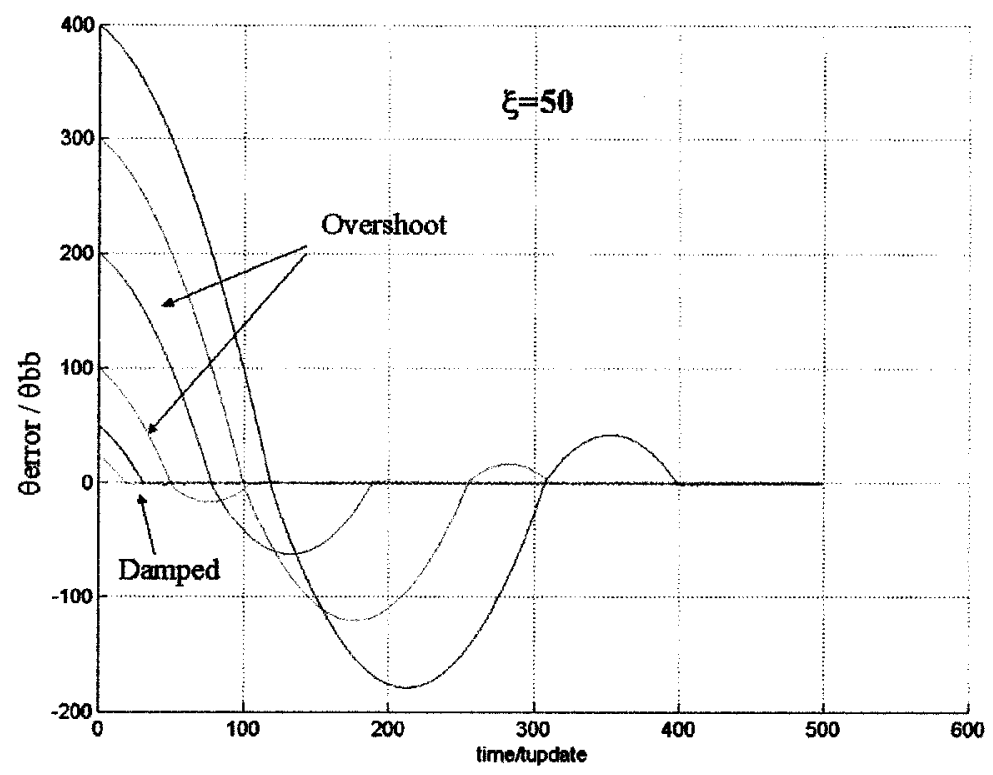

Figure 4.16 Simulated response of second order loop to phase step with range of input phase step amplitudes

The second order loop response of input phase step can be considered as a summing output signals of two separate loops: a proportional loop and an integral loop, as shown in Figure 4.17. Whenever the loop has a relatively low stability factor, the output phase is mainly the result of the integral loop where the effect of the proportional loop is insignificant. The integral loop experiences an oscillatory idling pattern as discussed earlier. Recall that the initial idling order mode can be determined from Equation 4.50. Therefore

$$
m_{\text {idling }}=2 \cdot \sqrt{\xi \cdot \frac{\theta}{\theta_{b b}}}
$$




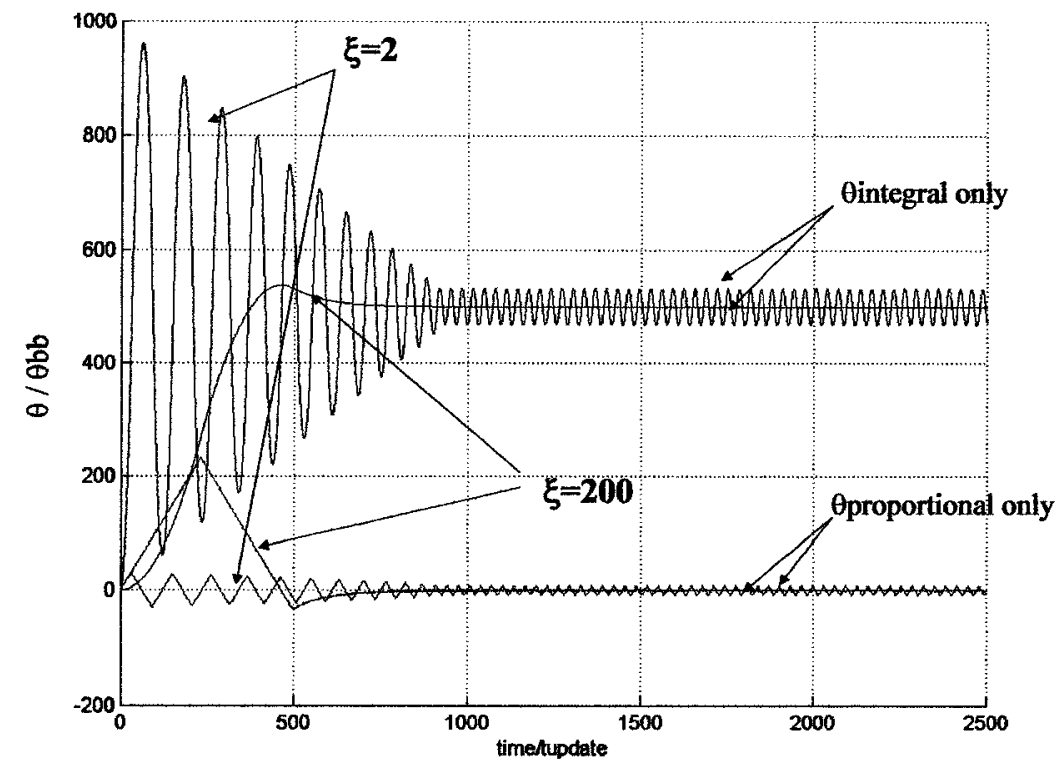

Figure 4.17 Simulated integral and proportional responses of second order loop to phase step with range of stability factors

The output phase due to the integral loop attempts to reach its peak value after $\left(m_{\text {idling }}\right) / 2$ cycles. The proportional loop attempts to build an output phase that is equal to $\left(\theta_{b b} m_{\text {idling }}\right)^{/ 2}$ which reduces the net phase error to $\theta-\left(\theta_{b b} m_{\text {idling }}\right) / 2$ at time $\left(t_{n} m_{\text {idling }}\right) / 2$, hence reducing the idling order mode (Equation 4.2). Consequently, the idling mode oscillation amplitude starts gradually to decrease at each idling mode period until $m_{\text {idling }}=1$ or $m_{\text {idling }}=2$.

If the loop has a relatively high stability factor, both proportional and integral responses will initially exhibit a slope overload. The proportional loop will attempt to build a large output phase which is enough to force the integral loop to have $m_{\text {idling }}=1$ or 
$m_{\text {idling }}=2$ within the first idling mode period. Afterwards, the proportional loop output phase starts to vanish.

\subsection{Frequency Step Response}

In order to get the frequency step response for the first order bang-bang loop, the loop is assumed to have $\theta_{d}=\delta \omega t \Upsilon(t)$ and $\phi=0$, where $r(t)$ is the unit step function at $t=0$. A Matlab model follows the diagram shown in Figure 4.10 is utilized to simulate the loop response and validate the equations. From Equation 4.29, the maximum input frequency step has to be bounded by the frequency change

$$
\delta \omega \leq\left|\omega_{b b} \cdot\left[1+\frac{2 m}{\xi}\right]\right|
$$

Thus,

$$
-\omega_{b b}\left\langle 1+\frac{2 m}{\xi}\right) \leq \delta \omega \leq \omega_{b b}\left\langle 1+\frac{2 m}{\xi}\right\rangle
$$

The second order loop has an advantage of wider lock range by a factor of $1+\frac{2 m}{\xi}$ than the first order loop. The widest lock range absorbed in one update time occurs when the second order loop has the minimum stability factor, which is three times larger than the first order loop lock range. If $\delta \omega$ falls behind $\omega_{b b} \cdot\left[1+\frac{2}{\xi}\right]$, then the loop will experience transient phase error. If the transient phase error exceeds the dynamic range of the phase detector, which is often $\mp \pi$, the loop experiences a cycle slipping and goes temporarily out of lock. When the loop is cycle slipping, the integral loop constantly pushes the center frequency of the VCO with delta frequency equaling $\frac{2}{\xi} \omega_{b b}$ every update period, 
until the frequency offset between the incoming data frequency and VCO center frequency comes within $\pm \omega_{b b}$ range. At this point, the proportional loop generates a DC voltage proportional to the offset frequency and pushes the VCO center frequency further towards the incoming data frequency as shown in Figure 4.18.

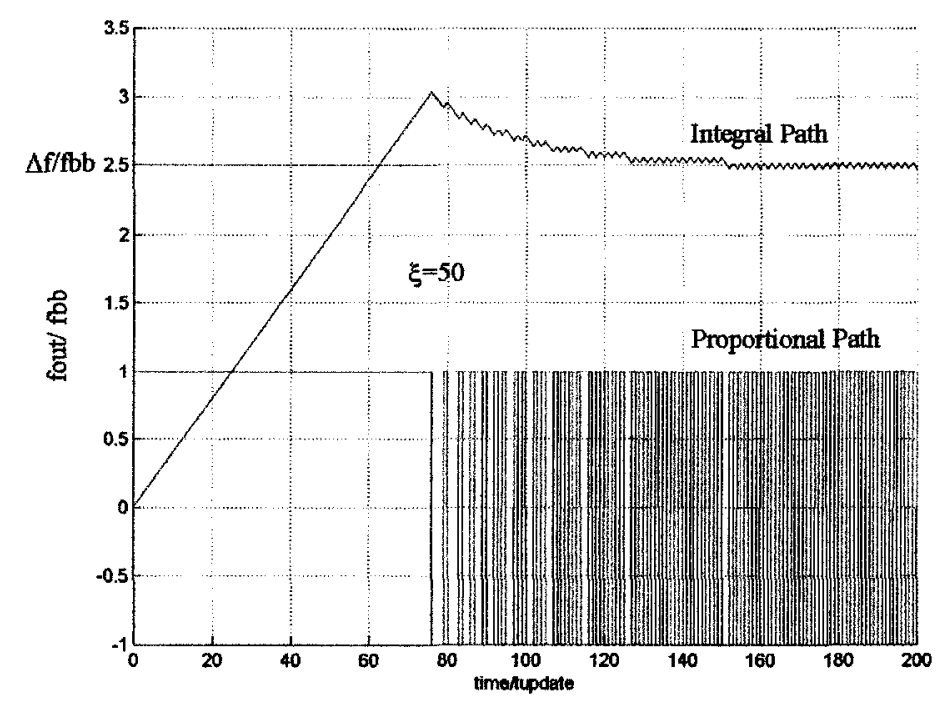

Figure 4.18 Simulated integral and proportional response of the second order loop to a frequency step

The number of cycles $m p$ needed by the integral loop to reduce the frequency offset to be within $\mp \omega_{b b}$ range at the maximum transient phase error $\left.\theta_{e r r o r}\right|_{p}$ is given by

$$
m_{p}=\frac{\xi}{2} \cdot\left[\frac{\delta \omega}{\omega_{b b}}-1\right]
$$


The lock-in-frequency time $\left(t_{p}\right)$ is then,

$$
t_{p}=\frac{\pi \xi}{\omega_{n}} \cdot\left[\frac{\delta \omega}{\omega_{b b}}-1\right]
$$

having

$$
\theta_{\text {error }}\left(m_{p}\right)=\theta_{d}\left(m_{p}\right)-\theta_{v}\left(m_{p}\right)
$$

Substituting the value of $\theta_{v}$ from Equation 4.28 and putting $\theta_{d}\left(m_{p}\right)=\delta \omega m_{p} t_{n}$, we get

$$
\frac{\theta_{\text {error }}\left(m_{p}\right)}{\theta_{b b}}=\left[\frac{\delta \omega}{\omega_{b b}}-1\right] \cdot m_{p}-\frac{m_{p}^{2}}{\xi}
$$

Substituting the value of $m_{p}$ from Equation 4.58 into 4.60 gives the maximum normalized transient phase error

$$
\left.\frac{\theta_{\text {error }}}{\theta_{b b}}\right|_{\text {peak }}=\left[\frac{\delta \omega}{\omega_{b b}}-1\right]^{2} \cdot \frac{\xi}{4}
$$


Figure 4.19 shows an agreement with Equation 4.58 and Equation 4.62. Whenever the second order loop has a higher stability factor, the loop causes higher phase error for same frequency step input and requires longer time to regain lock For example, for input $\frac{\delta \omega}{\omega_{b b}}=2.5$ and $\xi=40$, the maximum phase error of $22.5 \theta_{b b}$ occurs at $30 t_{n}$, and for $\xi=50$, the maximum phase error of $28.125 \theta_{b b}$ occurs at $37.5 t_{n}$.

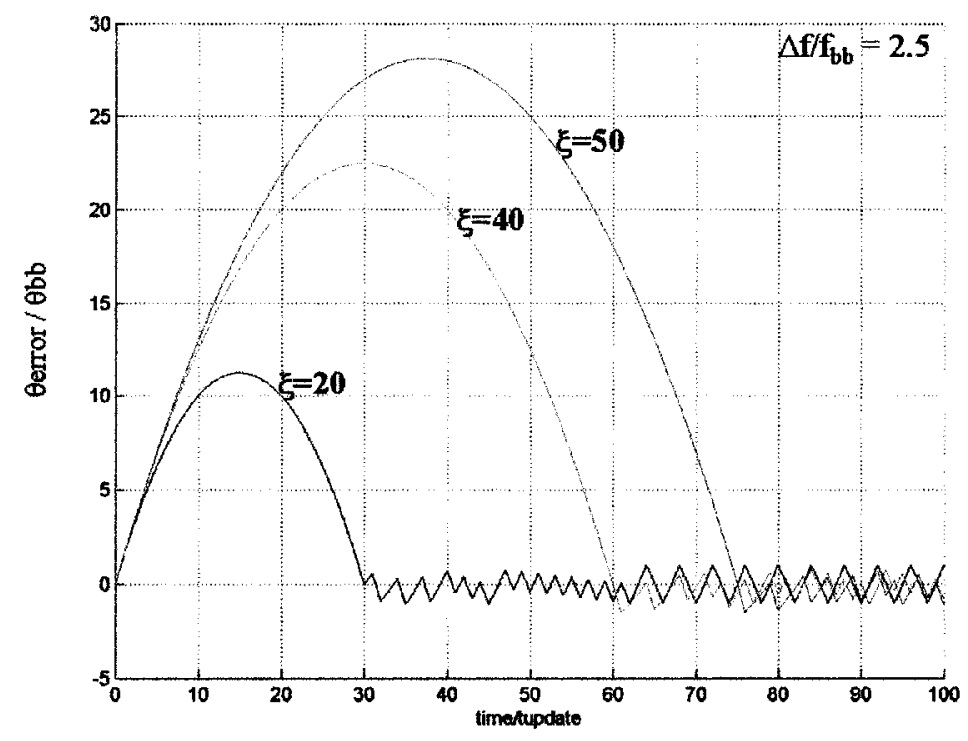

Figure 4.19 Simulated response of the second order loop to a frequency step with range of stability factors

In order to maintain the second order loop phase locked to the input frequency step, certain conditions have to be met. To stabilize the loop, the stability factor has to satisfy the condition in Equation 4.4. As well, the maximum phase error has to be bounded 
by the dynamic range boundary of the phase detector to avoid cycle slipping and stay phase locked. The maximum stability factor to avoid cycle slipping can be found by equating Equation 4.62 to $\pi$, then

$$
\xi_{\max }=\frac{4 \pi}{\theta_{b b} \cdot\left[\frac{\delta \omega}{\omega_{b b}}-1\right]^{2}}
$$

Therefore,

$$
\frac{m_{i}}{m_{i}-2} \leq \xi \leq \frac{4 \pi}{\theta_{b b} \cdot\left[\frac{\delta \omega}{\omega_{b b}}-1\right]^{2}}
$$

The simulated response of the second order loop in Figure 4.20 shows an agreement to Equation 4.63. The loop experiences instability when $\xi=1$ or $\xi=0$. For $\xi=2$, the loop has the fastest response and it absorbs the frequency hit (step) within one update time without any transient phase error and hence the loop stays phase locked. Furthermore, increasing the stability factor causes the transient phase error to increase till it falls beyond the dynamic range boundary of the phase detector, and hence the loop experiences cycle slips and goes out of lock. 


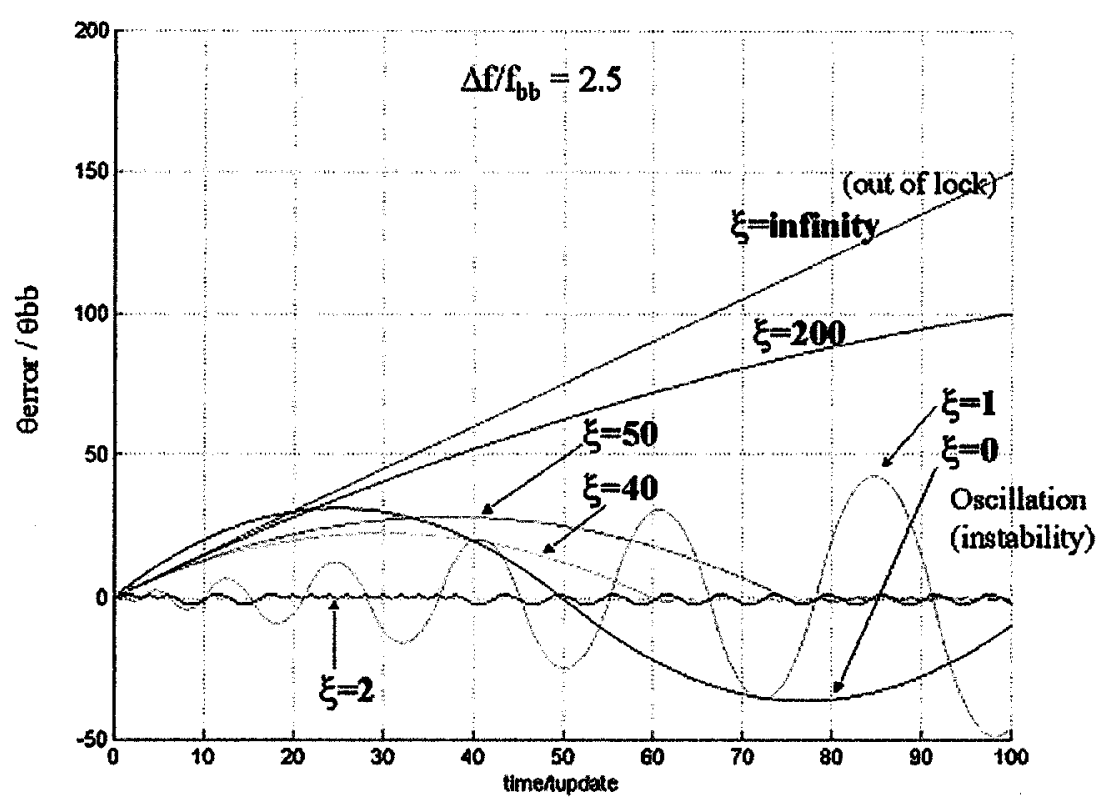

Figure 4.20 Simulated response of the second order loop to a frequency step with range of stability factors

Unlike the first order loop, the lock range of the second order loop has another dimension of design freedom by adjusting $\xi$ in addition to $\theta_{b b}$

\subsection{Sinusoidal Operating Conditions}

The analysis of the sinusoidal operating conditions assumes the loop is phase locked, that there is no frequency offset between the incoming data rate and the VCO frequency $(\delta \omega=0)$, and that $\phi=m_{j} \sin \left(\omega j \cdot t+\varphi_{o}\right)$, where

- $m_{j}$ is the input jitter amplitude in rad

- $\omega_{j}$ is the jitter angular frequency in $\mathrm{rad} / \mathrm{sec}$

- $\varphi_{o}$ is a zero mean phase shift.

From Equation 4.37, the maximum jitter amplitude that can be tracked without slope overload is 


$$
m_{\text {jmax }}=\frac{\omega_{b b}}{\omega_{j}}\left[1+\frac{\omega_{n}}{\pi \xi \omega_{j}}\right]
$$

Consequently, the maximum input frequency at a given input jitter amplitude (the corner frequency) when the loop tracks the input jitter can be expressed as

$$
\omega_{c} \equiv \omega_{j \max }=\frac{\frac{\omega_{b b}}{m_{j}}+\sqrt{\left(\frac{\omega_{b b}}{m_{j}}\right)^{2}+4 \cdot \frac{\omega_{b b} \omega_{n}}{\pi \xi m_{j}}}}{2}
$$

A Matlab model follows the diagram shown in Figure 4.9 is used to simulate the loop response and validate the equations. Figure 4.21 shows the loop behaves like a single pole system with a corner frequency equal to $\omega_{c}$ and the transfer function falls with almost $20 \mathrm{~dB}$ per decade. The corner frequency illustrated in the simulated waveforms is in satisfactory agreement with Equation 4.66, where the corner frequency is inversely proportional to the root square of the stability factor when the loop has a relatively low stability factor, and with almost no correlation when the loop has a relatively high stability factor. 


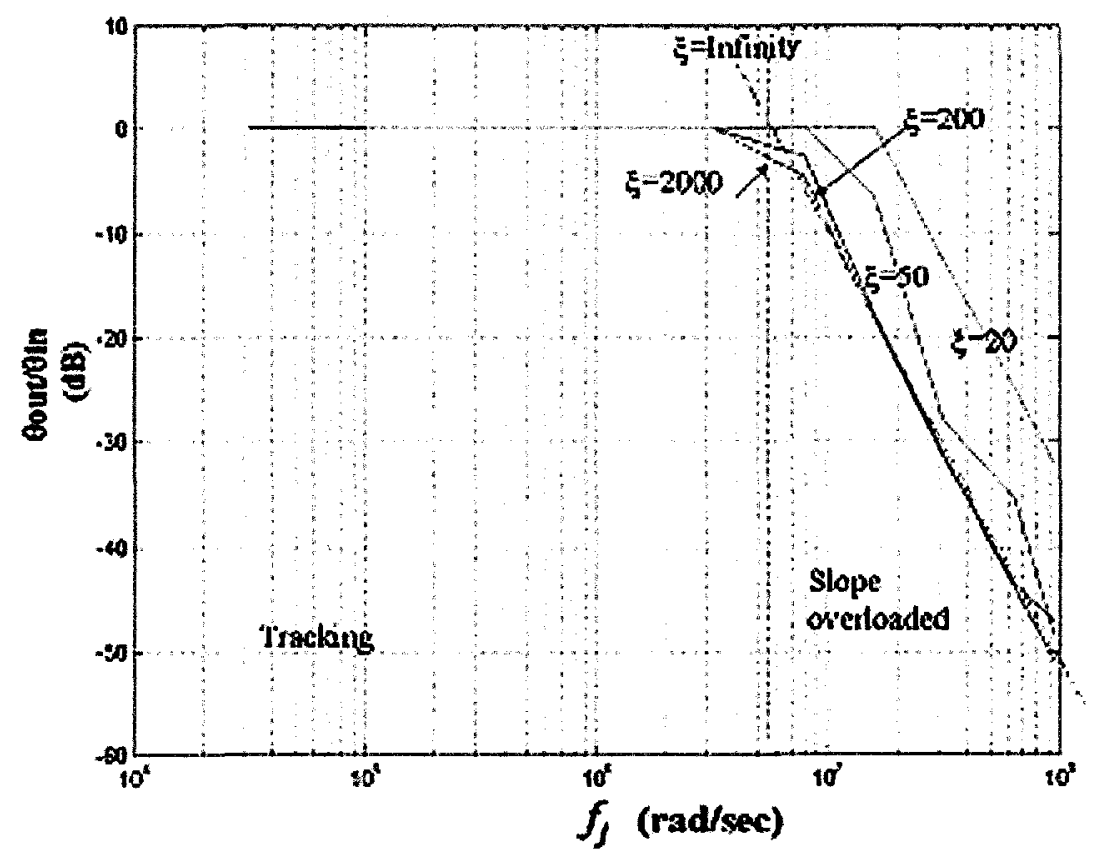

Figure 4.21 Simulated response of second order loop to a sinusoidal jitter with range of stability factors

Similar to the first order loop, the phase error of the second order loop acts as a single pole high pass filter with cut-off frequency equal to $\omega_{j \max }$. The loop has to have high ${ }^{\omega_{b b}}$ or high stability factor, to reduce transient phase error.

\subsection{Quantization Noise}

The technique discussed in chapter 2 to calculate the quantization noise is used in this section to develop the expression of signal-to-quantization noise ratio. 
We can use a similar reasoning to that in Section 3.6. That is, the power spectrum $S_{n n}(f)$ is flat over the jitter band and the quantization noise $N_{q}{ }^{2}$ is proportional to $f j b$. As well as the clock rate $f n$ increases, the quantization error is improved linearly with $f n$. Hence, we obtain $N_{q}{ }^{2} \propto 1 / f_{n}$. Consequently, $N_{q}$ depends on the size of step of $\theta_{v}(0)$. From Equation 4.13, we obtain

$$
\begin{gathered}
\theta_{v}(0) \propto \theta_{b b} \cdot \frac{f_{j b}}{f_{n}} \\
N_{q}{ }^{2}=C_{d} \cdot\left(\frac{f_{j b}}{f_{n}}\right)^{3} \cdot \theta_{b b}^{2}
\end{gathered}
$$

where $C_{d}$ is an empirical constant and with analogy to Double Delta Modulation, we get $20<C_{d}<54[44]$

\subsubsection{SQNR of DJ}

For sinusoidal input jitter with amplitude $m j$, the input power is $m_{j}^{2} / 2$ and the SQNR is given by

$$
S Q N R=\frac{1}{2 C_{d}}\left(\frac{f_{n}}{f_{j b}}\right)^{3}\left(\frac{m_{j}}{\theta_{b b}}\right)^{2}
$$

Since the quantization noise power $N_{q}^{2}$ is independent of the jitter power $s^{2}$, then SQNR is linearly proportional to $s^{2}$ and has maximum value when $s^{2} \mid \max =\left(\operatorname{m}_{j \max }\right)^{2} / 2$. 
Substituting the value of $m_{j \max }$ from Equation 4.65 into Equation 4.69 gives

$$
S Q N R_{\max }=\frac{1}{8 \pi^{2} C_{d}} \cdot \frac{f_{n}^{5}}{f_{j b}^{3} \cdot f_{j}^{2}} \cdot\left[1+\frac{f_{n}}{\pi \cdot \xi \cdot f_{j}}\right]^{2}
$$

The second order loop shows a significant conditional improvement of SQNR over the first order loop by a factor of $\delta S Q N R$, where $\delta S Q N R$ is the ratio between SQNR of the second order loop to the SQNR of the first order loop,

$$
\delta S Q N R=10 \cdot \log \left(\frac{1}{60}\right)+20 \cdot \log \left(\frac{f_{n}}{f_{j b}}\right)+20 \cdot \log \left(1+\frac{f_{n}}{f_{j}} \cdot \frac{1}{\pi \xi}\right)
$$

For example, the maximum bandwidth for the CDR in SONET OC-192 standard is $8 \mathrm{MHz}$, therefore,

$$
\frac{f_{n}}{f_{j}}=\frac{D F \cdot f_{o}}{B W} \approx \frac{0.5 \cdot 10 G H z}{B W}
$$

and assuming unfiltered jitter, then $\frac{f_{n}}{f_{j b}}=2$, therefore

$$
\delta S Q N R=10 \cdot \log \left(\frac{1}{15}\right)+20 \cdot \log \left(1+\frac{5 x 10^{9}}{\pi B W \xi}\right)
$$

Therefore, in case of unfiltered jitter, the second order loop improves the SQNR over the first order loop for the $\xi$ obtained within a range 


$$
1<\xi<\frac{D F \cdot f_{o}}{9 \cdot \omega_{c}}
$$

However, the SQNR of the second order loop is reduced and the second order loop experiences increased unfiltered noise when the stability factor falls beyond $\xi>\frac{D F \cdot f_{o}}{9 \cdot \omega_{c}}$, as shown in Figure 4.22. For example, for $8 \mathrm{MHz}$ and $4 \mathrm{MHz}$ loop bandwidth, the second order loop has a maximum improvement of $40 \mathrm{~dB}$ and $35 \mathrm{~dB}$ respectively for a minimum stability factor of one. The loop becomes noisy as long as the stability factor is greater than 75 and 150 for $8 \mathrm{MHz}$ and $4 \mathrm{MHz}$, respectively. This phenomenon explains why the second order loop might have a jitter peaking at higher stability factor.

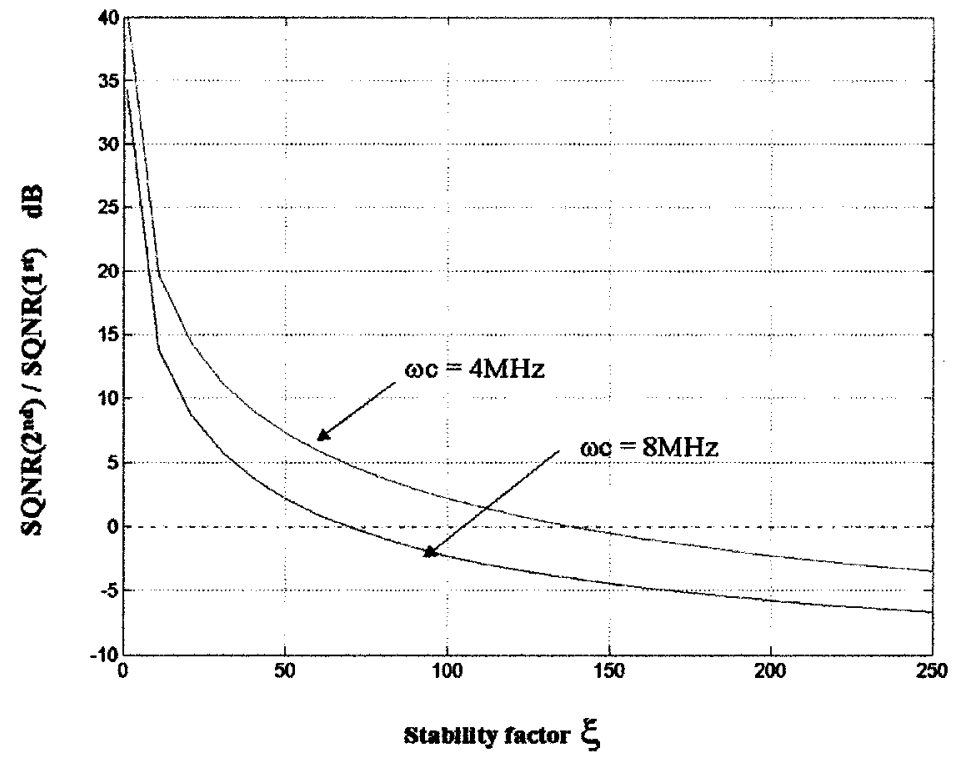

Figure 4.22 Normalized SQNR of second order loop response to stability factor with range of loop bandwidth when the input is deterministic jitter 
Nearly all high speed digital communication standards require the loop generated noise to be measured within a limited band. The second order loop shows unconditional superior improvement for band limited noise (filtered jitter), regardless of the value of the loop stability factor or the input jitter frequency. The limited band has be less than $\frac{f_{n}}{2 \sqrt{15}}$ or approximately $25 \%$ of total unfiltered jitter band.

\subsubsection{SQNR of RJ}

For Gaussian input jitter with variance $\sigma$, the input power is $\sigma^{2}$ and the SQNR is expressed as

$$
S Q N R=\frac{1}{C_{d}} \cdot\left(\frac{f_{n}}{f_{j b}}\right)^{3} \cdot\left(\frac{\sigma}{\theta_{b b}}\right)^{2}
$$

Since the quantization noise power $N_{q}{ }^{2}$ is independent of the jitter power $s^{2}$, the SQNR is linearly proportional to $s^{2}$ and has a maximum value when $s^{2} \mid \max =\sigma_{\max }{ }^{2}$. Substituting for the value of $\sigma$ from Equation 4.49 into 4.75 gives the maximum SQNR for Gaussian noise as

$$
S Q N R_{\max }=\frac{1}{4 \pi^{2} C^{2} C_{d}} \cdot \frac{f_{n}^{5}}{f_{j b}^{7}} \cdot\left(\sqrt{5} \frac{1}{\pi \xi}+\sqrt{3} f_{j b}\right)^{2}
$$

where $\mathrm{C}$ is the confidence level defined by Equation 3.13. From Equation 3.25 and 4.76, the $\delta S Q N R$ is given as 


$$
\delta S Q N R=\frac{K_{q}}{C_{d}} \cdot \frac{f_{n}^{2}}{f_{j b}^{4}} \cdot\left(\sqrt{5} \frac{1}{\pi \xi}+\sqrt{3} f_{j b}\right)^{2}
$$

Then,

$$
\delta S Q N R=10 \cdot \log \left(\frac{1}{60}\right)+20 \cdot \log \left(\frac{f_{n}}{f_{j b}}\right)+20 \cdot \log \left(\sqrt{3}+\frac{1}{f_{j b}} \cdot \frac{\sqrt{5}}{\pi \xi}\right)
$$

Assuming unfiltered jitter with $\frac{f_{n}}{f_{j b}}=2$,

$$
\delta S Q N R=10 \cdot \log \left(\frac{1}{15}\right)+20 \cdot \log \left(\sqrt{3}+\frac{\sqrt{5}}{f_{j b} \pi \xi}\right)
$$

Figure 4.23 shows that for Gaussian input jitter, the second order loop generates more unfiltered noise than the first order loop with a noise $7 \mathrm{~dB}$, regardless of the bandwidth of the loop and BER. However, it improves the SQNR over the first order loop for the filtered noise if the noise band is limited to be less than $44 \%$ of the unfiltered noise band. 


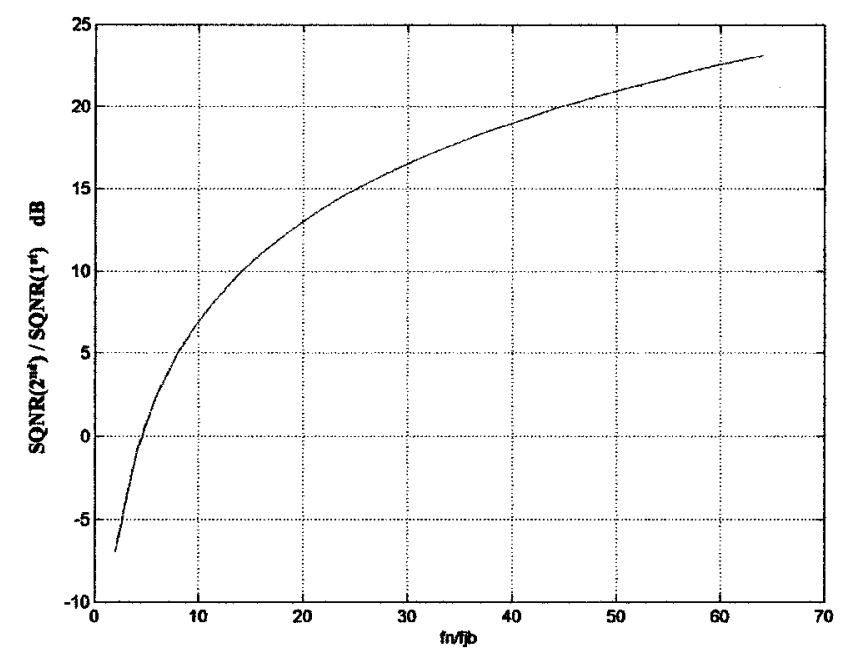

Figure 4.23 Normalized SQNR of second order loop response to ratio of $\mathrm{fn} / \mathrm{fjb}$ when the input is random jitter

\subsection{Jitter Analysis of Second-Order Loop}

Whenever the jitter performance of the second order loop is analyzed, the loop is assumed to have phase locked, to not be in a slope overload condition and to not frequency offset between the incoming data frequency and VCO frequency. A Matlab model follows the diagram shown in Figure 4.9 will be used for simulation of jitter performance and validating the equations.

\subsubsection{Jitter Transfer}

Like the first order loop, the second order loop has a jitter transfer function of a single pole low pass filter with a corner frequency given by Equation 4.65 , and by definition $D F=1$. Then the second order loop JTR is given by 


$$
J T R=\frac{\omega_{c}}{s+\omega_{c}}
$$

The bandwidth of the loop can therefore be rewritten as

$$
B W \equiv \omega_{c}=\frac{\frac{\beta K_{v}}{m_{j} \cdot f_{o}}+\sqrt{\left(\frac{\beta K_{v}}{m_{j} \cdot f_{o}}\right)^{2}+8 \cdot \frac{\beta K_{v} f_{o}}{\xi m_{j}}}}{2}
$$

Figure 4.24 shows that the simulated transfer function of the second order loop is in agreement with the Equation 4.82 .

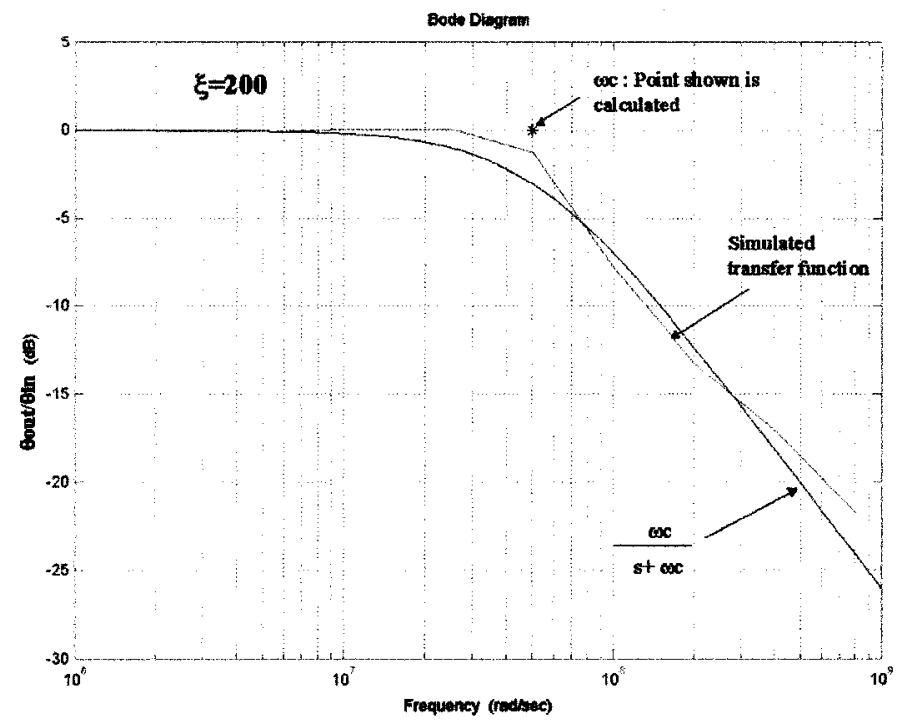

Figure 4.24 Simulated jitter transfer function of second order bang-bang loop

The jitter peaking of the second order loop $(J P)$ for sinusoidal input jitter is defined as 


$$
J P=10 \cdot \log \left(\frac{1}{S Q N R}\right) \quad[d B]
$$

Substituting with the value of SQNR from Equation 4.70 gives

$$
J P=10 \cdot \log \left(2 C_{d}\right)+30 \cdot \log \left(\frac{f_{i b}}{f_{n}}\right)+20 \cdot \log \left(\frac{m_{j}}{\theta_{b b}}\right)
$$

The maximum jitter peaking of the second order loop occurs when $f_{j b}=0.5 \cdot f_{n}$, therefore

$$
J P=6.99-20 \cdot \log \left(\frac{m_{j}}{\theta_{b b}}\right)
$$

According to the standard definition considered in chapter 2, jitter peaking has to be less than $0.1 \mathrm{~dB}$ for all ranges of frequency. Thus, the condition to satisfy the jitter peaking can be expressed as

$$
m_{j} \geq 2.21 \cdot \theta_{b b}
$$

Consequently, the required value $\theta_{b b}$ to meet jitter peaking is

$$
\theta_{\left.b b\right|_{J P}} \leq \frac{m_{j \min }}{2.21}
$$

where $m_{j \min }$ is the minimum input jitter defined by the standards. 


\subsubsection{Jitter Tolerance}

Similar to the discussion in Section 3.11.2, in order to achieve jitter tolerance for a targeted BER, the condition of slope overload of deterministic jitter over jitter frequency band has to be met in addition to the incoming random jitter, which must be bounded so that the loop can have better BER than the target BER.

The jitter tolerance spectrum due to deterministic jitter can be expressed using the slope overload definition from Equation 4.65. Replacing $\omega_{j}$ by ' $\mathrm{s}$ ', we obtain

$$
m_{\text {jmax }}=\frac{4 \pi \theta_{b b}}{\xi} \cdot \frac{1}{s} \cdot\left[\frac{\frac{\pi \xi}{\omega_{n}} \cdot s+1}{s}\right]
$$

The maximum tolerated sinusoidal input jitter has the characteristic of a single integrator which falls off at $20 \mathrm{~dB}$ per decade, followed by an integrator with phase lead correction with zero at $s=\frac{\omega_{n}}{\pi \xi}$. It has a Bode plot as shown in Figure 4.25. 


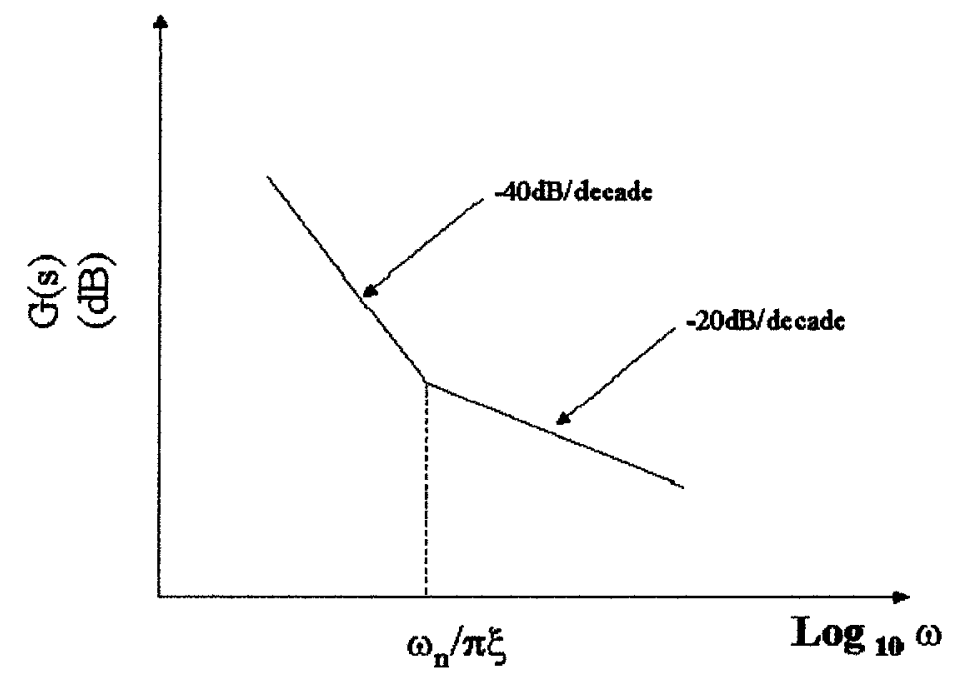

Figure 4.25 Bode plot of jitter tolerance of second order loop

Figure 4.26 shows the spectrum of a sinusoidal jitter which is just sufficient to cause slope overload with a range of stability factors. The second order loop with lower stability factor is capable of tolerating more input jitter than that with a higher stability factor. 


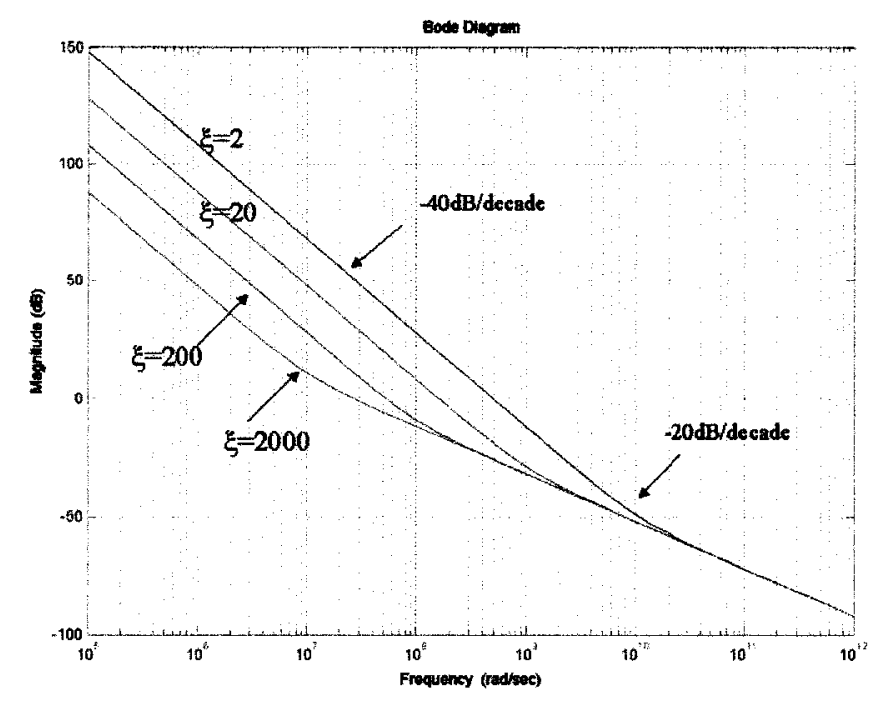

Figure 4.26 Jitter tolerance spectrum of second order loop to deterministic jitter with range of stability factors

The loop tolerates low frequency jitter better than high frequency. Therefore, the bang-bang phase $\left.\theta_{b b}\right|_{J T L}$ required to meet the deterministic jitter tolerance condition (so the loop is not overloaded) is given by

$$
\left.\theta_{b b}\right|_{J T L} \geq\left. m_{j \max }\right|_{\circlearrowleft j \max } \cdot \omega^{2}{ }_{j \max } \cdot\left(\frac{\frac{\xi}{4 \pi}}{\frac{\pi \xi}{\omega_{n}} \omega_{j m a x}+1}\right)
$$


where $\left.m_{j \max }\right|_{\omega j \max }$ is the maximum jitter amplitude at the maximum jitter frequency defined by jitter tolerance mask. In order to meet the jitter tolerance mask, the random jitter of the incoming data and the clock signal have to be bounded so as not to cause a slope overload at a target bit-error-rate better than $B E R=10^{-10}$. Therefore, from Equation 4.47 we get

$$
\left.\theta_{b b}\right|_{J T L} \geq \frac{2 \pi \cdot C \cdot \sigma \cdot \omega^{2}{ }_{j b}}{\omega_{n} \cdot\left(\frac{2 \sqrt{5}}{\xi}+\sqrt{3} \omega_{j b}\right)}
$$

Putting $C=\sqrt{42}$,

$$
\left.\theta_{b b}\right|_{J T L} \geq \frac{2 \pi \cdot \sqrt{42} \cdot \sigma \cdot \omega^{2}{ }_{j b}}{\omega_{n} \cdot\left(\frac{2 \sqrt{5}}{\xi}+\sqrt{3} \omega_{j b}\right)}
$$

The second order loop has to have a bang-bang step phase satisfying both Equations 4.88 and 4.89 in order to meet the jitter tolerance mask.

\subsubsection{Jitter Generation}

The jitter generation (TJ) is defined as the total output jitter when there is no input jitter. It is comprised of deterministic jitter due to the idling pattern of the loop, and random jitter associated with the clock signal edges. The idling pattern (deterministic jitter) of the second order loop has amplitude and frequency that is a function of the stability factor as discussed in Section 4.3. With the assumption that the stability factor is sufficiently high $(\xi>3)$, all higher oscillatory idling order modes would be reduced to the lowest 
oscillatory order modes of $m=1$ or $m=2$. After settling down, the quantized phase error will acquire a 101010101 pattern or 1100110011 idling pattern. Therefore the maximum deterministic jitter can be given from Equation 4.28 as

$$
D J=2 \cdot \theta_{b b} \cdot\left[m+\frac{m^{2}}{\xi}\right]
$$

Putting $m=2$ and having $\xi>3$,

$$
D J=4 \cdot \theta_{b b} \cdot\left[1+\frac{2}{\xi}\right]
$$

As discussed in Section 3.11.3, the effective source of random noise within the loop bandwidth is both random noise associated with the clock signal, and incoming data stream edges. Notice that the VCO has minimum effect on the output jitter since the loop has a relatively large bandwidth. The condition to avoid the slope overload at a target BER to a Gaussian random jitter is given by Equation 4.47. Substituting $C=7.13$ for $B E R=10^{-12}$ we get

$$
\left.\theta_{b b}\right|_{J T L} \geq \frac{2 \pi \cdot 7.13 \cdot \sigma \cdot \omega^{2}{ }_{j b}}{\omega_{n} \cdot\left(\frac{2 \sqrt{5}}{\xi}+\sqrt{3} \omega_{j b}\right)}
$$

where

$$
\sigma=\sqrt{\sigma_{d a t a}^{2}+\sigma_{c l o c k}^{2}}
$$

where 
- $\sigma_{\text {data }}$ is the root mean square of data stream's random noise

- $\sigma_{\text {clock }}$ is the root mean square of clock signal's random noise.

Consequently, the jitter generation (TJ) is given by

$$
T J=4 \cdot \theta_{b b} \cdot\left[1+\frac{2}{\xi}\right]+2 \cdot Q_{B E R} \cdot R J
$$

Substituting $\mathrm{Q}_{\mathrm{BER}}=7.0345$ for $\mathrm{BER}=10^{-12}$, we get the jitter generation formula to be

$$
T J \approx 4 \cdot \theta_{b b} \cdot\left[1+\frac{2}{\xi}\right]+14 \cdot R J
$$

\subsection{Summary}

The second order loop was demonstrated instable without phase lead correction. The stability factor was defined as a function of the idling order mode, which helps to explain why the loop experiences a wander in the event of a long run pattern of the incoming data stream. Alternative models based on Double Delta Modulation and second order Sigma Delta Modulation system were presented. A set of equations and expression were developed to explain slope overload and signal-to-noise ratio of Gaussian input jitter at a given bit-error rate. Similar analysis has demonstrated the sinusoidal input jitter that helps explain the sinusoidal operating conditions. A complete analysis of phase step response and frequency step response with calculation of lock-in-phase and lock-in-frequency time was presented. The loop performance in terms of jitter transfer, jitter peaking, jitter tolerance and jitter generation was defined as a function of the loop design parameters. Time domain simulation and behavioral mode simulation were used to verify the validity of the analysis in the case of a 5 Gbps CDR. 
The second order loop gives the designer the flexibility of using two design parameters; the stability factor $\xi$ and bang-bang phase step $\theta_{b b}$. This enables us to de-couple the lock range from the loop jitter performance. The analysis demonstrated the second order loop has a wider lock range, conditionally superior jitter performance and longer settling time. 
This thesis developed, in considerable depth, mathematical analysis of first and second order bang-bang CDR. Literature research showed that the bang-bang CDR is the most suitable and widely used clock and data recovery circuit at very high-speed bit rates for its superior performance.

A thorough review of the previous work on the analysis of the dynamics of the bang-bang CDR reveals a lack of theory and mathematical driven analysis of the loop characteristics and parameters, due to having nonlinear phase detector and sampling in the system, which prevents the use of the PLL linear theory in analysis. The thesis developed a set of mathematical equations, inequalities and conditions to explain and characterize both first and second order bang-bang CDR.

The analysis was driven from two directions. The first was based on using the linear Delta Modulation and Sigma Delta Modulation to explain the first order bang-bang loop, and using the double linear Delta Modulation and second order Delta Sigma Modulation systems to analyze the second order bang-bang loop. The second direction was based on frequency domain analysis using linearized models for developing an expression of frequency step response for first order and second order loops. This allowed for deriv- 
ing expressions and sets of equations explaining the loop dynamics of the bang-bang CDR and loop jitter performance as a function of bit-error-rate. The analysis provides expressions and equations that can be used in designing and optimizing the bang-bang clock and data recovery parameters, in order to meet the high speed digital communication standard requirements. It is important to note that some of the expression and equations contradict one another and a compromise is usually needed.

The understanding of Bang-Bang CDR behavior and its relation to design parameters has been enhanced through following novel contributions:

- developed formulas of slope overload conditions to Gaussian random jitter, Equations 3.15 and 4.47, for first and second order bang-bang CDR respectively. To the author's best knowledge, the slope overload to random jitter contribution is not previously reported.

-developed formulas of signal-to-noise ratio (SQNR) to Gaussian random as a function of bit-error-rate, Equations 3.25 and 4.76, for first and second order bang-bang CDR respectively. To the author's best knowledge, the SQNR contribution is not previously reported.

-developed jitter peaking equations. Equations 3.44 and 3.48 are for first order bangbang CDR and Equations 4.83 and 4.86 are for second order bang-bang CDR. To the author's best knowledge, the jitter peaking equations for bang-bang CDR have not been reported before.

- defined conditions and formulas in order to meet jitter tolerance requirements due to random jitter, Equations 3.59 and 4.89 for first and second order bang-bang CDR respectively. To the author's best knowledge, the equations to bound the jitter tolerance due to random jitter have not been reported before.

-demonstrated how the second order loop has a significant conditional improvement of SQNR over the first order loop; Equations 4.71 and 4.77. Such comparison has not been reported. 
- developed equations and conditions to meet jitter transfer mask, Equations 3.42 and 4.81, for first and second order bang-bang CDR respectively. The jitter transfer functions equations developed in this report are in agreement with the previously reported measurements [21], developed from accurate discrete time difference equations and improve the approximated formula obtained by Razavi where he approximated the second order loop to first order [30].

- defined conditions and formulas in order to meet both DJ and RJ jitter generation requirements. Equations 3.61 and 3.63 are for first order bang-bang CDR and Equations 4.92 and 4.93 are for second order loop. The developed jitter generation equations in this report enhance the previous reported work by Salama [31] and Razavi [30]. Salama found equation for DJ jitter generation for first and second order loops using timing analysis method, however, Razavi discussed approximated formula for VCO jitter generation.

-defined conditions and formulas in order to meet jitter tolerance requirements to deterministic input jitter. Equations 3.53 and 3.54 are for first order bang-bang CDR and Equations 4.87 and 4.88 are for second order bang-bang CDR. The work developed for jitter tolerance in this report is improving the work reported by Razavi [30]. Razavi reported a jitter tolerance approximated formula for maximum tolerated input jitter.

-defined the stability conditions of second order loop as function of the idling order mode which used to explain why the loop experiences a wander in the event of long run pattern of the incoming data stream, Equations 4.4 and 4.64. Walker was first to define the stability factor and its minimum value required to stabilize the second order loop [19] and Salama validated Walker's result by using a timing analysis method. The work presented in this report expands the definition of minimum stability factor to be function of the idling order modes as well as define the upper bound of the stability factor as a function of input step frequency.

-Improved the published discrete time difference equations reported by Walker [1] to describe the second order loop dynamics; Equations 4.5, 4.6 and 4.13. 
- the second order loop phase step response was discussed in details and lock-in-phase equation and maximum transient phase error were developed; Equations 4.50, 4.51, 4.54, and 4.55. The developed equations in this report improve the equations reported by Salama [31] and enhance the result obtained by Walker [1]. Walker discussed briefly the phase step response for the second order loop.

- lock-in-frequency equation and maximum transient phase error were developed for the second order loop; Equations 4.57 and 4.60. The developed equations are further improvement to the work reported by Salama [31]. Salama analyzed the second order loop transient frequency error using a timing analysis technique.

-defined the lock dynamic range of the second order loop, Equation 4.57. The equations obtained in this report for first order dynamic range is in agreement with the reported equations by Walker [1].

The equations developed were analytically driven and verified with time domain simulation. The equations and inequalities were formulated to help meet the requirement specifications within the assumptions that have been made.

The research can be pursued further in a number of areas:

The proposed equations and inequalities were verified either analytically or with time domain simulation. A testing chip of bang-bang clock and data recovery running at $1 \mathrm{Gbps}, 5$ Gbps and possibly $10 \mathrm{Gbps}$ with different setting parameters can be developed to allow for experimental verification. 
Furthermore, the analysis can be extended to study the non-uniform sampling system, asymmetrical loop response, meta-stability of the phase detector effect, run length of the incoming data stream effect, and limited VCO bandwidth. As well, it is worthwhile to extend the analysis to study higher order loops in order to take the effects of parasitics into account.

The results demonstrated in this thesis show that an accurate model for building bang-bang CDR loops can be realized. The work is a step forward towards completing the analysis of the bang-bang CDR, with emphasis on jitter characterization as a function of the design parameters. The work explains many phenomena of the bang-bang CDR behavior and can be used to optimize and design high speed CDR to comply with tight jitter specifications. The accuracy of quantization noise formula over reduced bandwidth white noise needs to be examined. Furthermore, jitter generation due to Gaussian input jitter of the second order loop deserves further investigation. 


\section{References}

1. R. Walker, "Designing Bang-bang PLLs for Clock and Data Recovery in Serial Data Transmission System," Phase Locking in High Performance Systems, IEEE Press, pp 34-45, 2003.

2. K. Ishii, et al, "A jitter suppression technique for a $2.48832-\mathrm{Gb} / \mathrm{s}$ clock and data recovery circuit," IEEE Transactions on Circuits and Systems II: Analog and Digital Signal Processing, vol. 49, no. 4, pp. 266-272, Apr. 2002.

3. D. Chen," A power and area efficient CMOS clock/data recovery circuit for high speed serial interfaces," IEEE, Journal of Solid-State Circuits, vol.31, no. 8, pp. 11701176, Aug. 1996.

4. T. Suzaki, et al., "Si bipolar chip set for $10 \mathrm{~Gb} / \mathrm{s}$ optical receiver," IEEE Journal of Solid-State Circuits, vol. 27, no. 12, pp. 1781-1786, Dec., 1992.

5. R. C. Walker, et al., "A 10Gb/s Si-Bipolar TX/RX Chipset for Computer Data Transmission," IEEE, International Solid-State Circuits Conference, pp. 302-303, 1998.

6. Y. M.Greshishchev, et al., "A $60 \mathrm{~dB}$ Gain $55 \mathrm{~dB}$ Dynamic Range $10 \mathrm{~Gb} / \mathrm{s}$ Broadband SiGs HBT Limiting Amplifier," IEEE, International Solid-State Circuits Conference, pp. 382-383, 1999.

7. L. Ingmar Andersson, et al, "Silicon Bipolar Chipset For SONET/SDH $10 \mathrm{~Gb} / \mathrm{s}$ FiberOptic Communication Links," IEEE Journal of Solid-State Circuits, vol. 30, no. 3, pp. 210-218, March., 1995.

8. T. Masuda, et al, " $40 \mathrm{~Gb} / \mathrm{s}$ Analog IC Chipset for Optical Receiver using SiGe HBTs," IEEE, International Solid-State Circuits Conference, pp. 19.7-1-19.7-3, 1998. 
9. A. Pttbacker, et al., "A Si bipolar Phase and Frequency Detector IC for Clock Extraction up to 8Gb/s," IEEE Journal of Solid-State Circuits, vol. 27, no. 12, pp. 1747-1751, Dec., 1992.

10. M. Meghelli, "SiGe BiCMOS 3.3V Clock and Data Recovery Circuits for $10 \mathrm{~Gb} / \mathrm{s}$ Serial Transmission Systems," IEEE, Journal of Solid-State Circuits, vol.35, no. 12, pp. 1992-1995, Dec.2000.

11. J. Wight,"Phase Locked-Loops and Receiver synchronizers," Carleton University, Course Notes 97.566, 2002.

12. Y. M.Greshishchev, "SiGe clock and data recovery IC with linear type PLL for $10 \mathrm{~Gb} /$ s SONET Application," IEEE, Journal of Solid-State Circuits, vol. 35, no. 9, pp. 13531359, Sept., 2000.

13. C. R. Hogge, "A Self Correcting Clock Recovery Circuit," IEEE, Journal of Lightwave Technology, vol. LT-3, pp. 1312-1314, Dec. 1985.

14. B. Razavi, "Design of Integrated Circuit for Optical Communications," New York, MacGraw-Hill 2003.

15. D. Shin," Self-correcting Clock Recovery Circuit with Improved Jitter Performance," Electronic Letters, vol. 23, No.3, pp.110-111, Jan. 1987.

16. L. M. De Vito, "Phase Detector For Phase Locked Loop Clock Recovery System," US patent no. 5,027,085, June, 1991.

17. J. Savoj, et al, "10 Gb/s CMOS Clock and Data Recovery Circuit with a Half Rate Linear Phase Detector," IEEE, Journal of Solid-State Circuits, vol. 36, no. 5, pp. 761768, May, 2001.

18. Y. Ohtomo, "12.5 Gb/s CMOS BER Test Using a Jitter-Tolerant Parallel CDR,", IEEE, International Solid-State Circuits Conference, 2004.

19. R. C. Walker, “A Two-Chip 1.5GBd Serial Line Interface," IEEE, Journal of SolidState Circuits, vol.27, pp.1805-1811, Dec.1992.

20. Y. M. Greshishchev, "SiGe Clock and Data Recovery IC with Linear-Type PLL for 10Gb/s SONET Application," IEEE, Journal of Solid-State Circuits, vol. 35, no. 9, pp. 1353-1359, Sept., 2000.

21. Y. M.Greshishchev, "A Fully integrated SiGe Receiver IC for $10 \mathrm{~Gb} / \mathrm{s}$ Data Rate," IEEE, International Solid-State Circuits Conference, pp. 52-53, Feb., 2000. 
22. H.S. Muthali, “A CMOS 10-Gb/s SONET Transceiver," IEEE, Journal of Solid State Circuits," vol. 39, No. 7, July 2004.

23. R. Payne, “A 6.25Gb/s Binary Adaptive DFE with First Post-Cursor Tap Cancellation for Serial Backplane Communications," IEEE, International Solid-State Circuits Conference, pp.68-69, 2005.

24. N. Krishnapura, et al," $A$ 5Gb/s NRZ Transceiver with Adaptive Equalization for Backplane Transmission," IEEE, International Solid-State Circuits Conference, pp.60$61,2005$.

25. M. Sorna, et al," A $6.4 \mathrm{~Gb} / \mathrm{s}$ CMOS SerDes Core with Feedforward and DecisionFeedback Equalization,", IEEE, International Solid-State Circuits Conference, pp.62$63,2005$.

26. J. D. Alexander, "Clock recovery from random binary signals," Electron. Letter, vol. 11, pp.541-542, Oct. 1975.

27. SONET OC192, "Transport system generic criteria," Bellcore, GR-1377-CORE, no.4, Mar. 1998.

28. "CEI Electrical and Jitter Interoparability Agreements for $6+$ and $11+\mathrm{Gb} / \mathrm{s} \mathrm{I} / \mathrm{O}$," Optical Internetworking Forum, July 2004.

29. J. Lee, et al, "Modeling of Jitter in Bang-Bang Clock and Data Recovery Circuits," IEEE, Custom Integrated Circuit Conference, pp 711-714, 2003.

30. J. Lee, "Analysis and Modeling of Bang-Bang Clock and Data Recovery Circuits," IEEE, Journal of Solid-State Circuits, vol.39, pp.1571-1580, Setp.2004.

31. M. Ramezani, "Analysis of a Half-Rate Bang-Bang Phase Locked Loop", IEEE, Transactions on Circuits and System-II, Analog and Digital Signal Processing, Vol. 49, No. 7, July 2002.

32. M. Ramezani, "Jitter Analysis of a PLL-based CDR with a Banag-Bang Phase Detector," MWSCAS, volume 3, pp. 393 -396 vol.3, Aug. 2002.

33. M. Ramezani, “An improved Bang-bang Phase Detector for Clock and Data Recovery Applications," 2001 IEEE International Symposium, vol.1, pp.715-718, May 2001.

34. Y. Choi, et al, "Jitter Transfer Analysis of Tracked Oversampling Techniques for Multigigabit Clock and Data Recovery", IEEE, Transaction on Circuits and System-II, Analog and Digital Signal Processing, vol. 50, No. 11, Nov 2003. 
35. N. Da Dalt, “A Design-Oriented Study of the Nonlinear Dynamic of Digital BangBang PLLs", IEEE, Transactions on Circuits and System-I, Regular papers, vol. 52, No. 1, Jan. 2005.

36. K. Vichienchom, "Analysis of Phase noise due to Bang-Bang Phase detector in PLLbased Clock and Data Recovery Circuits", IEEE, Circuits and Systems, vol.1, pp. I617-I-620, May 2003.

37. T. H. Toifl, et al, "Analysis of Parameter-Independent PLLs with Bang-Bang Phase Detectors," IEEE International Conference on Electronics, Circuits and Systems, vol. 2, pp.299-302, Sept. 1998.

38. H. Nosaka, et al, "A 10Gb/s Data-Pattern Independent Clock and Data Recovery Circuit with a Two Mode Phase Comparator," IEEE, Journal of Solid-State Circuits, vol. 38, no. 2, pp. 192-197, Feb.2003.

39. F. G. Gardner, "Charge-pump phase-lock loops," IEEE, Transaction on communications, vol.-COM-28, no. 11, Nov. 1980.

40. B. Razavi, "Phase-Locking in High-Performance Systems From Devices to Architectures," IEEE PRESS, 2003.

41. B. P. Lathi, “Communication System,” John Wiley \& Sons, 1968.

42. A. Blanchard, "Phase-Locked Loops: Applications to Coherent Receiver Design," Wiley 1979.

43. G. I. Bourdopoulos, "Dela-Sigma Modulators: Modeling, Design and Applications," London, Imperial College Press, 2003

44. R. Steel, Delta Modulation Systems. New York/Toronto: Wiley 1975.

45. J. C. Candy, “Oversampling Delta-Sigma Data Converters," IEEE PRESS, pp. 1-25 1992. 